Aus der Abteilung Präventive Zahnmedizin, Parodontologie und Kariologie (Komm. Direktor: Prof. Dr. med. dent. M. Hülsmann) im Zentrum Zahn- , Mund- und Kieferheilkunde der Medizinischen Fakultät der Universität Göttingen

\title{
In- vitro- Untersuchung der Effektivität verschiedener Lacke zur Prävention von White- Spot- Läsionen im Rahmen kieferorthopädischer Behandlungen
}

\author{
INAUGURAL - DISSERTATION \\ zur Erlangung des Doktorgrades für Zahnheilkunde \\ der Medizinischen Fakultät der Georg- August- Universität zu Göttingen
}

\author{
vorgelegt von \\ Tessa Kroker \\ aus \\ Meppen
}


Dekan:

1. Berichterstatter:

2. Berichterstatter/-in:

3. Berichterstatter/-in:

Tag der mündlichen Prüfung: $\quad$ 13.05.2013

I
Prof. Dr. rer. nat. H. K. Kroemer

Prof. Dr. med. dent. R. F. Mausberg

Prof. Dr. med. dent. D. Kubein-Meesenburg 


\section{Inhaltsverzeichnis}

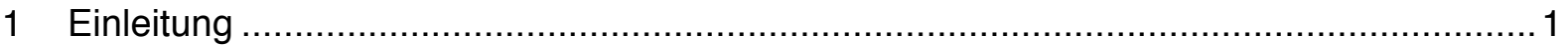

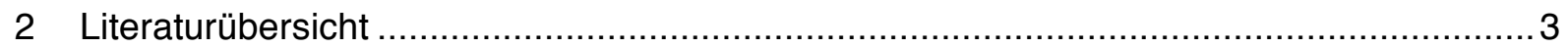

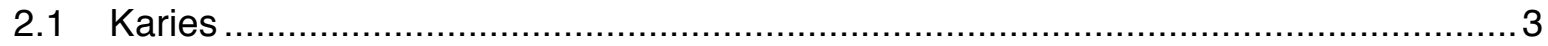

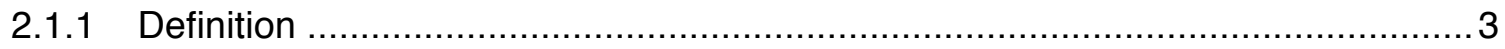

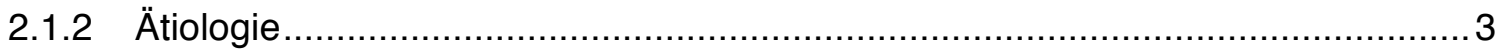

2.1.3 Struktur und Stoffwechsel der Plaque ............................................................. 4

2.1.4 Stellenwert von Streptococcus mutans bei der Kariesentstehung ...................... 4

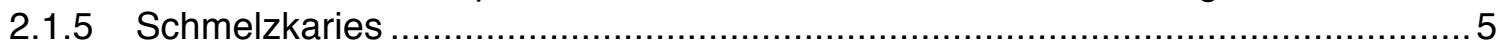

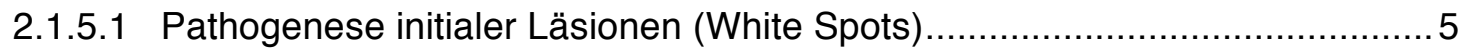

2.1.5.2 Histologie der Schmelzkaries ............................................................ 6

2.2 Demineralisationsrisiko im Rahmen kieferorthopädischer Behandlungen .................7

2.2.1 Inzidenz von White- Spot- Formationen .................................................. 7

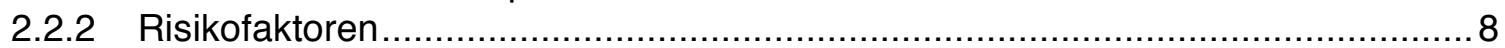

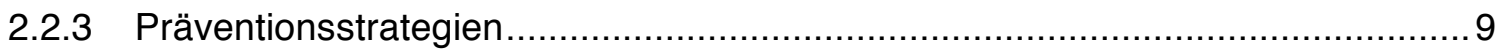

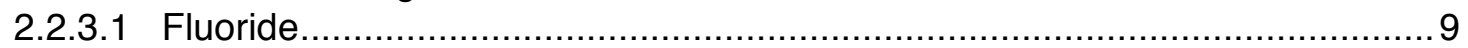

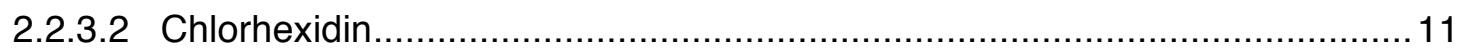

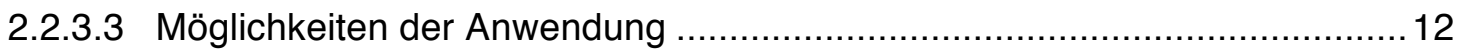

2.3 Studienlage zur Erzeugung von Demineralisationen in vitro .................................. 14

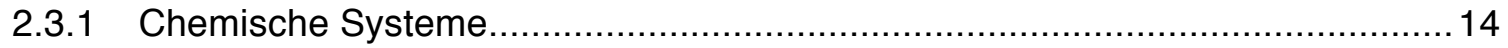

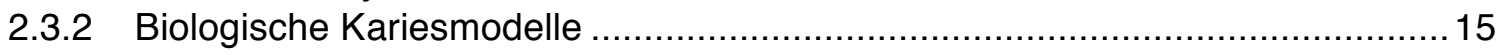

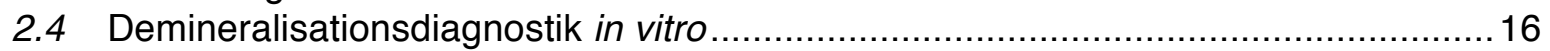

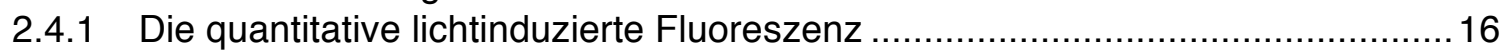

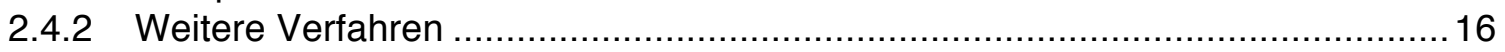

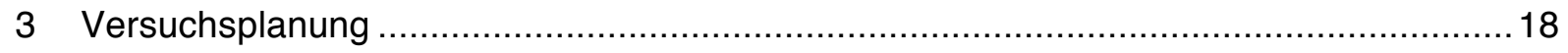

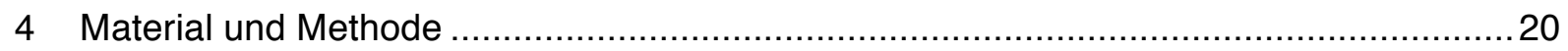

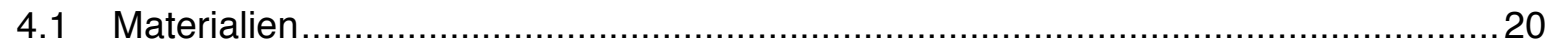

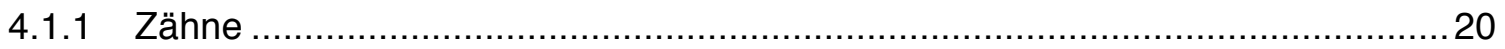

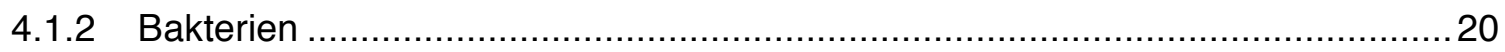

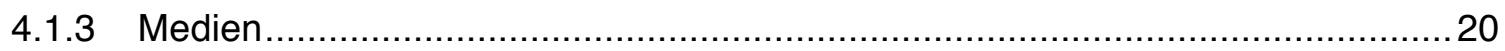

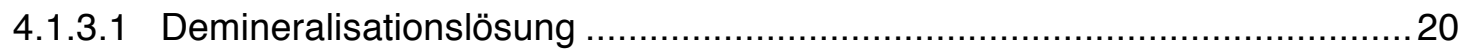

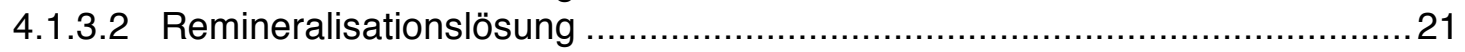

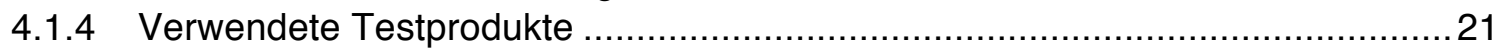

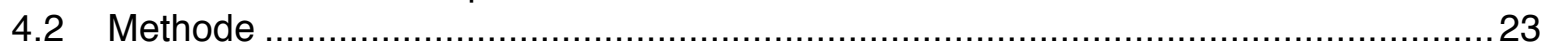

4.2.1 Herstellen der Prüfkörper und Zuweisung in Gruppen .................................23

4.2.2 Vorkultivierung der Bakterien und Ansetzen der Bakterienlösung .....................26

4.2.3 Messungen und Analyse mittels quantitativer lichtinduzierter Fluoreszenz .......28

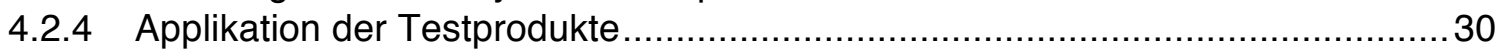

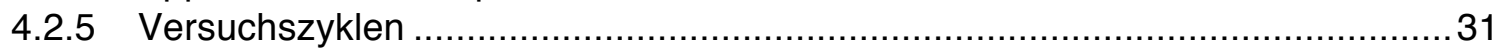

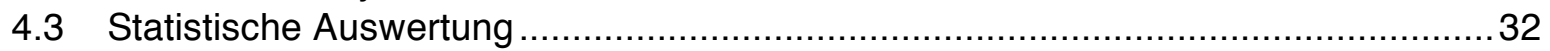

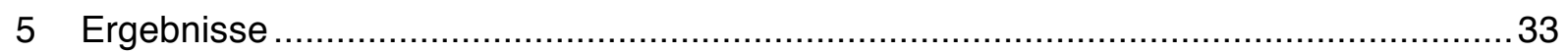

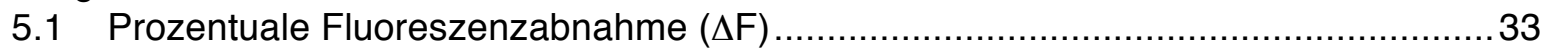

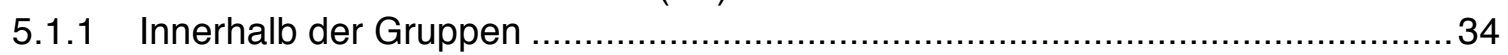

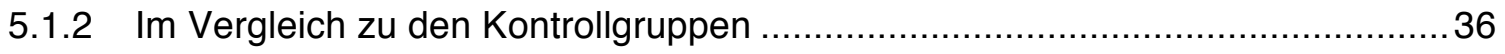

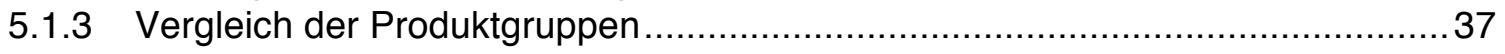

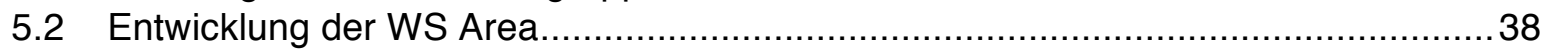

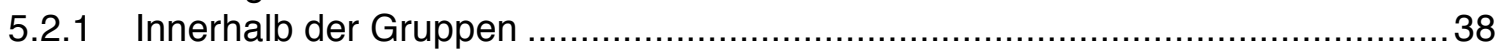

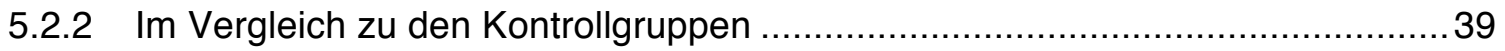




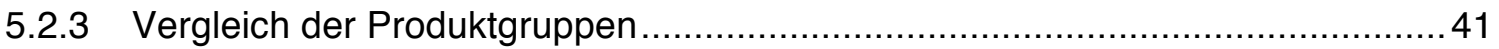

5.3 Korrelation von prozentualer Fluoreszenzabnahme $(\Delta \mathrm{F})$ und WS Area ..................41

5.4 Zusammenfassung der wichtigsten Ergebnisse .............................................. 43

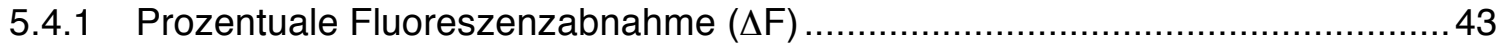

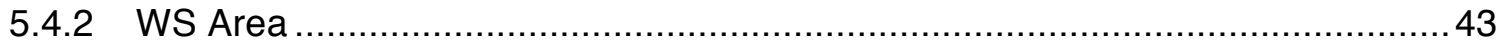

5.4.3 Korrelation von prozentualer Fluoreszenzabnahme und WS Area ....................44

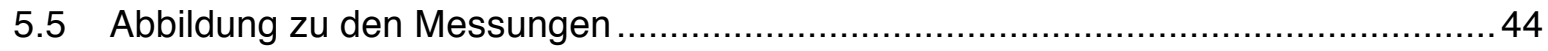

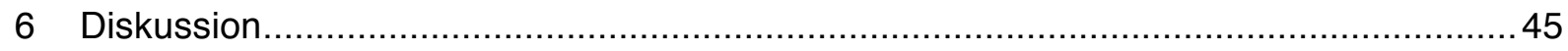

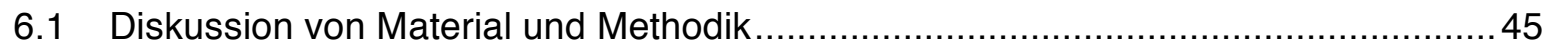

6.1.1 Beurteilung des verwendeten in- vitro- Kariesmodells .................................. 45

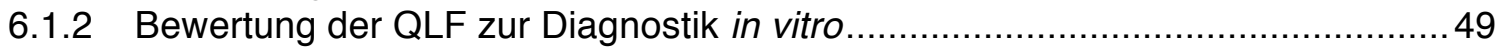

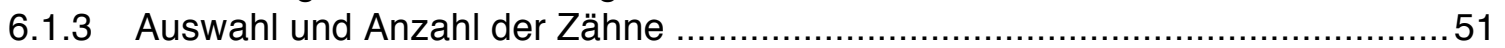

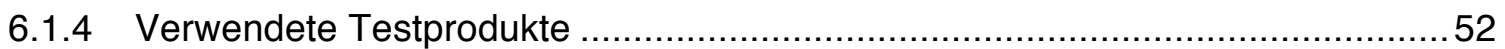

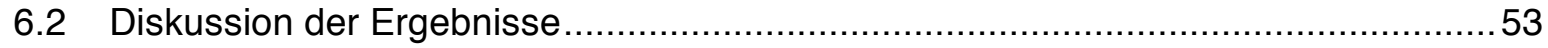

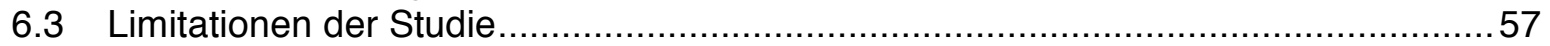

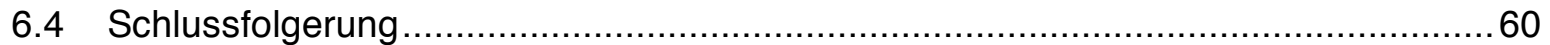

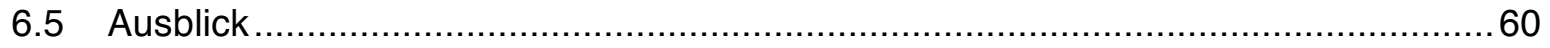

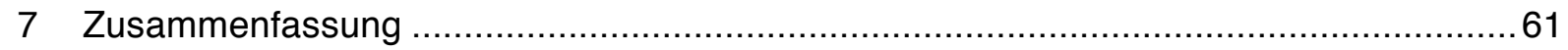

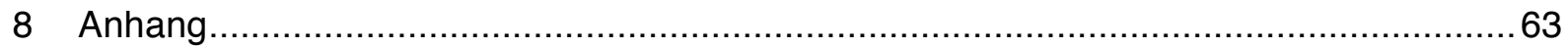

8.1 OD- Messwerte der eingesetzten Bakteriensuspensionen ...................................63

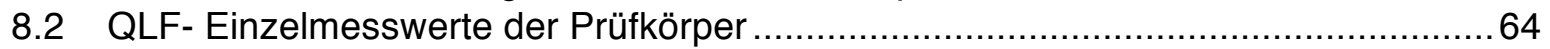

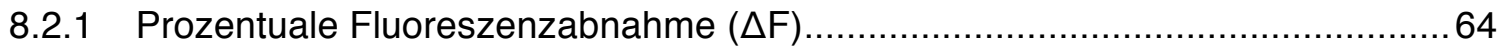

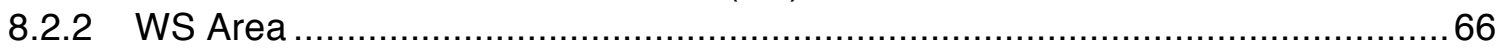

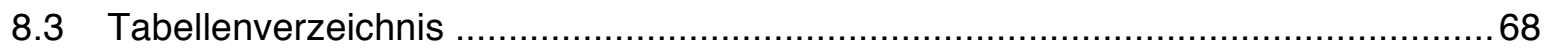

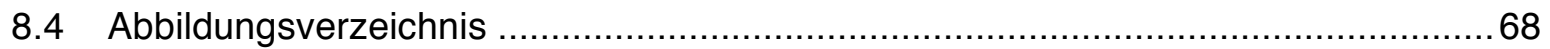

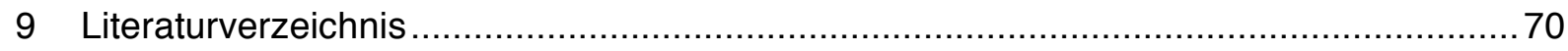




\section{Einleitung}

Kieferorthopädische Behandlungen werden mit dem Ziel durchgeführt, Kiefer- und Zahnfehlstellungen zu korrigieren sowie neben einer funktionell optimalen Einstellung des stomatognathen Systems auch den gestiegenen Ansprüchen einer harmonischen Ästhetik nachzukommen (Buren et al. 2008). Insbesondere durch den Einsatz festsitzender kieferorthopädischer Apparaturen können gezielte Zahnbewegungen durchgeführt und eine korrekte Ausformung der Zahnbögen erreicht werden. Während kieferorthopädischer Behandlungen mit festsitzenden Apparaturen ist jedoch die Mundhygiene erschwert; Brackets und Bänder begünstigen eine vermehrte Plaqueakkumulation, sodass ein erhöhtes Demineralisationsrisiko während der Behandlung besteht (Mitchell 1992, Zimmer und Rottwinkel 2004, Hadler-Olsen et al. 2011). So können im Rahmen kieferorthopädischer Behandlungen innerhalb eines kurzen Zeitraumes initiale Demineralisationserscheinungen des Schmelzes - sogenannte White- Spot- Läsionen - auftreten (O'Reilly und Featherstone 1987, Gorton und Featherstone 2003). Diese Läsionen sind oftmals irreversibel, neben ästhetischen Einbußen kann es zu einem Fortschreiten des initialen Demineralisationsprozesses und in der Folge zum Entstehen behandlungsbedürftiger Läsionen kommen. Diese Erkenntnisse implizieren, dass der frühzeitigen Erkennung und der Anwendung präventiver Maßnahmen besondere Beachtung geschenkt werden sollte, um das Demineralisationsrisiko im Rahmen der kieferorthopädischen Behandlung zu reduzieren und somit auch aus kariespräventiver und ästhetischer Sicht ein optimales Behandlungsergebnis zu erzielen (Tufekci et al. 2011).

Neben regelmäßigen Kontrollterminen, Ernährungsempfehlungen und wiederholten Mundhygieneinstruktionen können zusätzlich Produkte mit kariespräventiver Wirksamkeit Anwendung finden, um das Demineralisationsrisiko zu senken. Insbesondere der Einsatz fluoridhaltiger Produkte zeigte sich in klinischen Studien als vielversprechend (Gorton und Featherstone 2003, Ogaard et al. 2006, Lovrov et al. 2007, Bergstrand und Twetman 2011). Die topische Fluoridzufuhr kann dabei in Form von Mundspüllösungen, Gelen, Lacken und fluoridfreisetzenden Befestigungsmaterialien erfolgen (Pseiner 2008). Gerade bei Patienten mit geringer Compliance ist die Verwendung von Lacken von Vorteil, da diese professionell durch das Behandlungsteam eines Zahnarztes appliziert werden, über eine längere Wirkdauer verfügen und nicht von der Mitarbeit des Patienten abhängig sind (Kneist et al. 2008, Pseiner 2008).

Fluoridfreisetzende Versiegelungslacke können im Rahmen der Bracketadhäsivtechnik eingesetzt werden, um eine Demineralisation des Schmelzes im Bracketumfeld zu verhüten (Zachrisson 1976, Hu und Featherstone 2005). Insbesondere die Anwendung von lichthär- 
tenden Versiegelungslacken mit einem hohen Füllstoffgehalt stellt durch optimierte Polymerisation und erhöhte Abrasionsresistenz eine vielversprechende Maßnahme der Prävention von White- Spot- Läsionen dar (Hu und Featherstone 2005, Buren et al. 2008, Hess et al. 2011). Dennoch sind weitere Studien erforderlich, um einen Evidenznachweis zur Effektivität dieser Produkte zu erbringen (Keim 2011).

Die Eingliederung einer Multibandapparatur führt oftmals zu einem Anstieg von Streptococcus mutans und Laktobazillen und somit zu einem erhöhten kariogenen Milieu (Lundstrom und Krasse 1987). Daher können neben dem Einsatz von Fluoriden Produkte mit antimikrobieller Wirksamkeit zur Prävention Anwendung finden. Unter diesen Produkten wird Chlorhexidin als der Goldstandard angesehen, um eine Keimzahlreduktion sowie eine Plaquehemmung zu erzielen (Arweiler et al. 2011). Die nachhaltigste Keimreduktion wird erzielt, wenn $\mathrm{CHX}$ - haltige Lacke eingesetzt werden (Emilson 1994). Auch wenn eine tendenzielle Keimzahlreduktion durch $\mathrm{CHX}$ - haltige Produkte belegt werden konnte, ist deren Effektivität zur Prävention von White- Spot- Läsionen jedoch bisher wenig evidenzbasiert (Autio-Gold 2008).

Aufgrund einer Vielzahl verschiedener Lackanwendungsmöglichkeiten stellt sich die Frage, welche Anwendungsform einen effektiven Schutz gewährleistet, und ob Effektivitätsunterschiede zwischen verschiedenen Lackanwendungen bestehen. Ziel dieser Studie war es daher, die Effektivität verschiedener Lacke zur Prävention von White- Spot- Läsionen zu testen und zu vergleichen.

Um die Produkte unter kontrollierbaren Bedingungen testen zu können, bestand ein weiteres Ziel der Studie darin, ein biologisches Kariesmodell zu entwickeln, das eine der klinischen Situation ähnliche Demineralisationserzeugung in vitro ermöglicht. Aus den genannten Zielen ergaben sich damit folgende Arbeitshypothesen:

- Das eingesetzte Kariesmodell ermöglicht eine der klinischen Situation ähnliche Demineralisationserzeugung und stellt eine geeignete Methode dar, um Produkte mit kariespräventiver Wirksamkeit in vitro zu testen.

- Der Einsatz der getesteten Produkte führt zu einer effektiven Prävention von WhiteSpot- Läsionen.

- Es bestehen keine Effektivitätsunterschiede zwischen den getesteten Produkten. 


\section{Literaturübersicht}

\subsection{Karies}

\subsubsection{Definition}

Die Fédération Dentaire Internationale (FDI) definiert die Zahnkaries (lat. Caries „Morschheit“, „Fäulnis“, medizinisch Caries dentium) als einen „lokalisierte(n), pathologische(n) Vorgang (...) bakteriellen Ursprungs (...), der zur Entmineralisation der Zahnhartsubstanzen und schließlich zur Kavitätenbildung führt" (Franke und Baume 1976, S.77). Es handelt sich um eine weltweit verbreitete, unspezifische Infektionskrankheit der Zahnhartgewebe, die in unterschiedlichen Ausprägungsformen - von der klinisch nicht sichtbaren submikroskopischen Veränderung bis zu einer deutlich diagnostizierbaren Kavitation - manifest werden kann (Hellwig et al. 2009).

\subsection{2 Ätiologie}

Die 1889 von Miller beschriebene chemoparasitäre Theorie - Mikroorganismen bilden bei Zufuhr niedermolekularer Kohlenhydrate Säuren, die zu einer Demineralisation der Zahnhartgewebe führen können - bildet noch heute die Grundlage der Theorie der Kariesentstehung, wurde jedoch modifiziert und erweitert. Keyes (1962) benannte die Wirtsfaktoren sowie das Substrat und die Mikroorganismen als notwendige Faktoren der Kariesentstehung. König erweiterte 1971 die von Keyes beschriebenen Ätiologiefaktoren um den Faktor Zeit. Auch die Frequenz der Nahrungsaufnahme ist für die Kariesentstehung von Bedeutung (Gustafsson et al. 1954). Neben den vier genannten primären Faktoren nimmt eine Reihe von Sekundärfaktoren, beispielsweise das Gesundheitsverhalten, bisher unbekannte genetische Faktoren, aber auch sozioökonomische Komponenten auf den Prozess der Kariesentstehung Einfluss (Hellwig et al. 2009). Somit lässt sich die Karies nach heutigem Wissensstand als eine multifaktorielle Erkrankung beschreiben, wobei das Zusammenwirken aller Faktoren letztlich entscheidet, ob und in welchem Maße Karies entsteht (Buchalla 2008). 


\subsubsection{Struktur und Stoffwechsel der Plaque}

Der Begriff Zahnplaque beschreibt den komplexen Biofilm, der fest auf der Zahnoberfläche haftet und in dem dreidimensional organisierte Bakterienpopulationen in eine extrazelluläre Matrix aus Polymeren eingebettet sind (Marsh P und Martin 2003). Die Entwicklung vollzieht sich über mehrere Tage, sodass erst nach 7 - 14 Tagen von einer ausgereiften Plaque gesprochen wird. Der reife Biofilm umfasst ein komplexes System von bis zu 700 verschiedenen, konkurrierenden und symbiotisch koexistierenden Bakterienspezies, die in vielfältiger Weise miteinander interagieren, sodass die Eigenschaften eines Einzelorganismus in diesem Biofilm modifiziert und erweitert werden können (Aas et al. 2005, Marsh PD et al. 2011).

Die Kariogenität der Plaque hängt sowohl von der Struktur als auch von der mikrobiellen Zusammensetzung ab (Busscher und van der Mei 1997). In diesem Zusammenhang wurde die ökologische Plaquehypothese postuliert. Diese besagt, dass pathologische Prozesse wie Karies auftreten, wenn es aufgrund von Veränderungen der lokalen Umweltbedingungen zu einer Verschiebung im Gleichgewicht der residenten Mikroflora kommt. Wird durch häufige Zufuhr niedermolekularer Kohlenhydrate der $\mathrm{pH}$ - Wert in der Plaque über einen längeren Zeitraum gesenkt, kommt es zu einer Veränderung der mikrobiellen Zusammensetzung. Azidogene und säuretolerante Keime wie Streptococcus mutans oder Laktobazillen können besser an diese Umweltveränderung adaptieren und sind dadurch anderen Mikroorganismen kompetitiv überlegen. Es kommt zu einer Vermehrung dieser Bakterien und die Plaque entwickelt ein kariogeneres Potential (Marsh PD 1994, Marsh PD 2006).

\subsubsection{Stellenwert von Streptococcus mutans bei der Kariesentstehung}

S. mutans spielt eine maßgebliche Rolle bei der Initiation und Progression kariöser Läsionen und die korrelierende Prävalenz von Kariesanstieg und dem Auftreten von S.mutans wurde vielfach belegt (Orland et al. 1954, Orland et al. 1955, Fitzgerald und Keyes 1960, Keyes und Fitzgerald 1962, Krasse 1966, Chang et al. 1997). Dieser besondere Stellenwert als Kariesleitkeim beruht auf den Stoffwechselleistungen und einer Reihe von Virulenzfaktoren (Emilson und Krasse 1985). Durch enzymatische Spaltung von Saccharose werden extrazelluläre Polysaccharide synthetisiert, die eine feste Adhäsion an die Zahnoberfläche und die Etablierung einer kariogenen Plaque ermöglichen. Aus aufgenommenen Kohlenhydraten werden intrazelluläre Polysaccharide synthetisiert, die den Bakterien als Reservoir bei Substratmangel dienen. Zur Energiegewinnung werden aufgenommene Kohlenhydrate durch 
anaerobe Glykolyse zu organischen Säuren verstoffwechselt, die anschließend ausgeschleust werden und zu einer Demineralisation der Zahnhartsubstanz führen können (Hellwig et al. 2009). Neben ihren azidogenen Eigenschaften verfügen Mutans- Streptokokken über eine Säuretoleranz, die es innen ermöglicht, auch bei länger bestehendem niedrigen $\mathrm{pH}$ Wert metabolisch aktiv zu bleiben. Durch Ausschleusung von Protonen und sauren Endprodukten, durch vermehrte glykolytische Aktivität, durch Verschiebung des $\mathrm{pH}$ - Optimums für Glucosetransport, Glykolyse und Undurchlässigkeit von Protonen, durch gesteigerte Aktivität der $\mathrm{H}^{+} /$ATP- Synthase, durch Übergang zum homofermentativen Stoffwechsel sowie durch Bildung von Stressreaktionsproteinen kann die Stabilität des intrazellulären $\mathrm{pH}$ - Wertes aufrechterhalten werden (Marsh P und Martin 2003).

\subsubsection{Schmelzkaries}

Bei der Schmelzkaries handelt es sich um eine Läsion, die noch keine Kavitation aufweist und nur auf den Schmelz begrenzt ist. Klinisch stellt sich die initiale Läsion als opake weißliche Veränderung der Schmelzoberfläche (White- Spot- Läsion) dar (Schroeder 1997).

\subsubsection{Pathogenese initialer Läsionen (White Spots)}

Wenn niedermolekulare Kohlenhydrate aus der Nahrung in die Plaque diffundieren, werden diese von den Mikroorganismen verstoffwechselt, sodass es zur Bildung von Säuren und somit zu einem Abfall des pH- Wertes kommt (Stephan 1940). Fällt der pH- Wert unter den kritischen Bereich von 5,5 - 5,0, werden Mineralien aus dem Schmelzkristallgitter herausgelöst und wandern in die Plaque ab (Demineralisation). Durch den Speichel erfolgt in der Plaque eine rasche Neutralisation der Säuren, es kommt zu einem pH- Wert- Anstieg sowie einer umgekehrten lonenbewegung von der Plaque in den Schmelz (Remineralisation). Der ständige Wechsel zwischen De- und Remineralisation stellt einen wiederkehrenden dynamischen Prozess dar. Entsteht jedoch ein Ungleichgewicht zwischen De- und Remineralisation und die Demineralisation überwiegt, so wird der kariöse Prozess in Gang gesetzt. Bei fortbestehender Demineralisation entwickelt sich neben dem Mineralverlust an der Schmelzoberfläche ein Konzentrationsgradient im Schmelzinneren, der dazu führt, dass Mineralien aus den tieferliegenden Schichten des Schmelzes zur Oberfläche diffundieren und dort repräzipitieren; es entsteht eine pseudointakte Oberfläche. Bei noch vorhandener Oberflächenintegrität besteht die Möglichkeit einer Läsionsarretierung oder -remission; schreitet der kariöse 
Prozess hingegen fort, wird schließlich die Schmelz- Dentin- Grenze erreicht und es kommt zu einem Einbruch der Schmelzoberfläche, sodass ein irreversibler Kavitationsdefekt entsteht (Chang et al. 1997, Schroeder 1997).

\subsubsection{Histologie der Schmelzkaries}

Histologisch kann die Schmelzkaries in vier verschiedene Zonen unterteilt werden; von zentral nach peripher betrachtet sind dies die transluzente Zone, die dunkle Zone, das Zentrum der Läsion und die pseudointakte Oberflächenschicht (Abb.2.1).
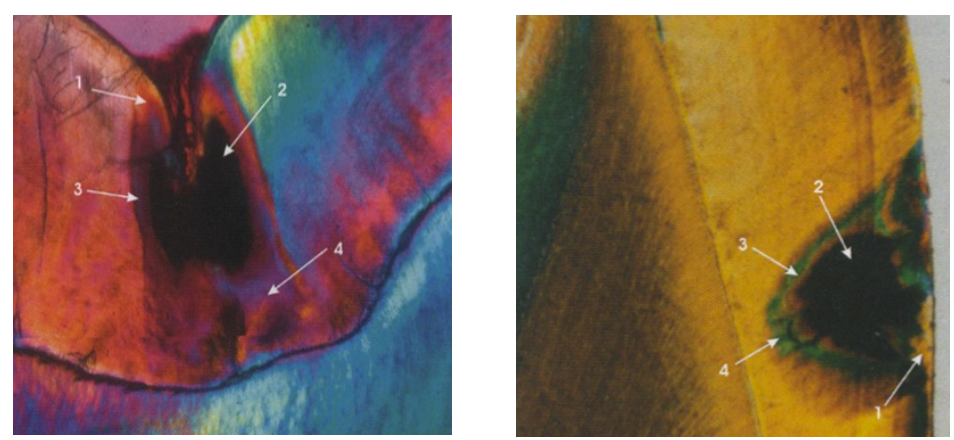

Abb.2.1 Polarisationsmikroskopisches Bild einer Schmelzkaries: 1) pseudointakte Oberflächenschicht, 2) Zentrum der Läsion, 3) dunkle Zone, 4) transluzente Zone (Gängler et al. 2010, S.120)

Die transluzente Zone im Schmelzinneren stellt die initialen im Lichtmikroskop erkennbaren Veränderungen des Kristallgefüges mit einem Porenvolumen von einem Prozent dar (das Porenvolumen von gesundem Schmelz beträgt 0,1\%). Die dunkle Zone besitzt ein Porenvolumen von 2-4\%. An die dunkle Zone schließt sich das Zentrum der Läsion an, es stellt die größte Zone innerhalb der Läsion dar. In dieser Zone findet sich der größte Mineralverlust, das Porenvolumen variiert von $5 \%$ in der Peripherie bis zu $25 \%$ oder mehr im Zentrum. Als vierte Zone lässt sich im Lichtmikroskop eine scheinbar intakte 20-50 $\mu$ m dicke Oberflächenschicht erkennen. Diese überdeckt die darunter liegenden Demineralisationsprozesse und entsteht durch Remineralisations- und Repräzipitationsvorgänge an der Schmelzoberfläche. Das Porenvolumen in dieser Zone beträgt weniger als 5\%, der Mineralverlust liegt bei 1-10\% (Silverstone 1981). 


\subsection{Demineralisationsrisiko im Rahmen kieferorthopädischer Be- handlungen}

\subsubsection{Inzidenz von White- Spot- Formationen}

Zahnfehlstellungen können die Mundhygiene erschweren, Retentionsnischen für Plaque bilden und somit die Entstehung kariöser Läsionen begünstigen. Demgegenüber ist jedoch gerade während der kieferorthopädischen Behandlung mit festsitzenden Apparaturen die Mundhygiene wiederum erschwert, sodass das Auftreten initialkariöser Läsionen (White Spots) ein klinisch relevantes Problem im Zuge der Behandlung darstellt (Gorelick et al. 1982, Mizrahi 1982, Lovrov et al. 2007).

Zahlreiche klinische Studien belegen einen deutlichen Anstieg des Auftretens von WhiteSpot- Läsionen (WSL) im Zuge kieferorthopädischer Behandlungen (Gorelick et al. 1982, Mizrahi 1982, Ogaard et al. 1988b, Mitchell 1992, Lovrov et al. 2007, Enaia et al. 2011, Richter et al. 2011). Zwischen den Studien variieren die Angaben über das Ausmaß des Anstieges zwischen 2 und 96 Prozent. Diese hohe Schwankungsbreite ist dadurch bedingt, dass bei den Studien stets individuelle Probandenparameter (Anzahl vorbestehender kariöser Läsionen, Ess- und Mundhygienegewohnheiten, Fluoridanwendung) Einfluss nehmen und die Erhebungsverfahren Unterschiede aufweisen (Mitchell 1992, Lovrov et al. 2007, Benham et al. 2009). So lag bei Enaia et al. (2011) die Inzidenz von WSL während der Multibandphase bei 60,9\% der Patienten; nach der Behandlung wiesen 63,3\% milde Läsionen auf und nur bei $26 \%$ aller Probanden waren keine neuen WSL während der gesamten Untersuchung zu verzeichnen. Richter et al. (2011) stellten fest, dass die Inzidenz mindestens einer neuen WSL während der Behandlung bei 72,9\% lag, 2,3\% darunter bereits mit Kavitation.

Das Auftreten neuer White Spots (Abb.2.2) lässt sich in besonderem Maße an den Vestibulärflächen der lateralen Inzisiven des Oberkiefers sowie den Eckzähnen und Prämolaren des Unterkiefers beobachten (Artun und Brobakken 1986, Lovrov et al. 2007). Chapman et al. (2010) zeigten in einer retrospektiven Studie, dass bei Patienten nach abgeschlossener kieferorthopädischer Behandlung in 36\% der Fälle mindestens eine White- Spot- Läsion auf der labialen Fläche der anterioren Oberkieferzähne vorlag, die Inzidenz der Oberkiefer- 2er lag bei $34 \%$, der 3er bei $31 \%$, der Prämolaren bei $28 \%$ und die Inzidenz der 1er bei $17 \%$. Auch wenn nach der Entbänderung eine Remineralisation aufgetretener White- Spot- Läsionen möglich ist, sind diese Läsionen oftmals irreversibel (Ogaard 1989). In einer Studie von Willmot (2004) wurde zwölf Wochen nach der Entbänderung eine Reduktion der LäsionsgröBen um ein Drittel und nach 26 Wochen eine Reduktion um die Hälfte bei den Teilnehmern 
der Studie festgestellt. Demgegenüber konstatierte Ogaard 1989, dass die White- Spot- Läsionen noch 5 Jahre nach abgeschlossener kieferorthopädischer Behandlung präsent waren.
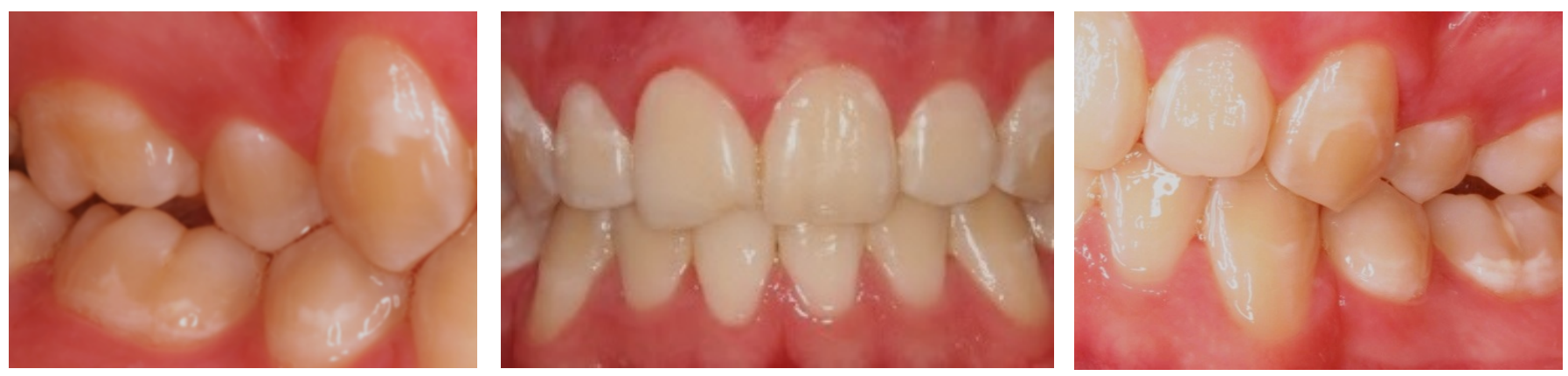

Abb.2.2 White- Spot- Läsionen eines kieferorthopädisch behandelten Patienten nach Entbänderung (Quelle: PD Dr. W. Hahn, Abteilung Kieferorthopädie des Zentrums für Zahn-, Mund- und Kieferheilkunde der Universitätsmedizin Göttingen)

\subsubsection{Risikofaktoren}

Patienten, die festsitzende Apparaturen tragen, besitzen ein signifikant höheres Demineralisationsrisiko als Patienten, bei denen keine Behandlung mit festsitzenden Apparaturen erforderlich ist (Gorelick et al. 1982, Hadler-Olsen et al. 2011).

Es gibt verschiedene Risikofaktoren, die das Auftreten von White Spots im Rahmen der Behandlung mit festsitzenden Apparaturen begünstigen. Durch Brackets, Ligaturen und Bänder ist die Mundhygiene erheblich erschwert. Sie stellen zugleich zusätzliche Retentionsnischen für die Plaque dar (Artun und Brobakken 1986). Dadurch ist eine vermehrte Plaqueakkumulation, insbesondere im Bereich zwischen Bracket und marginalem Gingivasaum, zu verzeichnen (Gorelick et al. 1982, Artun und Brobakken 1986). Darüber hinaus lässt sich auch eine Veränderung der Plaquezusammensetzung feststellen (Topaloglu-Ak et al. 2011). Es kommt zu einem Abfall des $\mathrm{pH}$ - Wertes und einem Anstieg von Streptococcus mutans und Laktobazillen, sodass die Plaque ein kariogeneres Potential entwickelt (Balenseifen und Madonia 1970, Lundstrom und Krasse 1987). Vor diesem Hintergrund stellt eine unzureichende Compliance einen Risikofaktor dar (Geiger et al. 1992, Hadler-Olsen et al. 2011). Studien konnten zeigen, dass eine Korrelation zwischen dem Auftreten von White Spots während der Multibandbehandlung und oralen Hygieneparametern besteht (Lovrov et al. 2007, Enaia et al. 2011). Des Weiteren wurde diskutiert, ob Geschlechtsspezifika vorliegen (Mizrahi 1982, Enaia et al. 2011, Tufekci et al. 2011). Während Enaia et al. und Tufekci et al. tendenziell schlechtere Werte bei den männlichen Probanden feststellten, beobachtete 
Mizrahi nur einen stärkeren Schweregrad der Opazitäten nach KFO- Behandlung bei den männlichen Probanden, die Prävalenz hingegen war bei beiden Geschlechtern nicht signifikant unterschiedlich. Inwieweit das Alter der Patienten während der Behandlung relevant ist, wurde ebenfalls kontrovers diskutiert. In Studien wurde sowohl ein tendenziell häufigeres Auftreten während der Pubertät als auch bei besonders jungen Patienten beschrieben. Bei der Erwachsenenbehandlung traten White Spots dagegen seltener auf (Kukleva et al. 2002, Enaia et al. 2011, Richter et al. 2011). Demgegenüber konnten Chapman et al. (2010) keine nachweisbare Korrelation von Alter und Auftreten initialkariöser Läsionen im Zuge der kieferorthopädischen Behandlung feststellen. Auch der mögliche Einfluss der Behandlungsdauer auf das Auftreten von White- Spot- Läsionen wurde untersucht. Es gibt Studien, die eine signifikante Korrelation zwischen längerer Behandlungsdauer und gehäufterem Auftreten von WSL belegen (Geiger et al. 1988, Richter et al. 2011). So stellten Geiger et al. in ihrer Studie fest, dass alle moderaten und ausgeprägten White- Spot- Läsionen bei den Probanden auftraten, deren Multibandapparatur mehr als 24 Monate in situ war. Tufekci et al (2011) beobachteten, dass vor allem in der initialen Behandlungsphase vermehrt WSL auftraten. So kann es bereits in den ersten vier Wochen der Behandlung mit festsitzenden Apparaturen zur Bildung erster WSL kommen (O'Reilly und Featherstone 1987, Ogaard et al. 1988a).

\subsubsection{Präventionsstrategien}

Ein konsequent durchgeführtes Prophylaxeregime senkt das Risiko der Entstehung von White- Spot- Läsionen (Zimmer und Rottwinkel 2004, Hadler-Olsen et al. 2011). Unerlässlich ist die Gewährleistung einer optimalen Mundhygiene (Kneist et al. 2008). Diese sollte in regelmäßigen Kontrollterminen während der gesamten Behandlungsdauer durch Remotivation und Reinstruktion des Patienten sichergestellt werden (Travess et al. 2004). Auch das Ernährungsverhalten sollte kritisch beurteilt und, falls nötig, umgestellt werden. Eine effektive Präventionsstrategie besteht in der zusätzlichen Verwendung von Produkten mit kariesprophylaktischer Wirksamkeit (Kneist et al. 2008, Bergstrand und Twetman 2011).

\subsubsection{Fluoride}

Fluoride nehmen einen zentralen Stellenwert in der Kariesprävention ein (Fischer et al. 1994, Bergstrand und Twetman 2011, Rao und Malhotra 2011). Die Applikation kann sowohl systemisch als auch lokal erfolgen, jedoch wirken Fluoride vorwiegend durch Kontakt mit Zahn- 
hartsubstanzen karieshemmend (ten Cate und Featherstone 1991, Hotz 1996). Aus diesem Grund sollten lokale Maßnahmen bevorzugt werden (DGZMK 2000). Nach heutigem Wissenstand geht man davon aus, dass die topische Wirkungsweise vorwiegend auf folgenden Mechanismen beruht:

\section{Hemmung der Demineralisation und Förderung der Remineralisation}

Der anorganische Bestandteil des Schmelzes besteht vorwiegend aus Hydroxylapatit. Dieses besitzt einen nicht- stöchiometrischen Aufbau und enthält oftmals Defekte und Fehlstellen in seinem Kristallgitter (Fischer et al. 1994). Ein Einbau von Fluorid in den Schmelz führt durch Bildung von Fluorapatit zu einer Verbesserung der Kristallinität und damit verbunden in geringem Ausmaß zu einer verminderten Säurelöslichkeit des Schmelzes (ten Cate und Duijsters 1983). Fluorapatit besitzt im Vergleich zu Hydroxylapatit ein niedrigeres Löslichkeitsprodukt. Während es bei einem pH- Wert von 5,5 bereits zu einer Untersättigung von Hydroxylapatit und in der Folge zu dessen Auflösung kommt, bleibt Fluorapatit unter diesen Bedingungen noch stabil (Hellwig 1996, Featherstone 2000). Es konnte jedoch gezeigt werden, dass gelöste Fluoridionen in der Zahnumgebung einen höheren Stellenwert in der Kariesprävention besitzen als strukturell gebundenes Fluorid (Ogaard et al. 1988c). Sind freie Fluoridionen während eines Säureangriffs in der flüssigen Phase des interkristallinen Zahnhartsubstanzbereiches vorhanden, wird die Säurelöslichkeit des Schmelzes herabgesetzt, indem die Fluoridionen an die Oberfläche der Hydroxylapatitkristalle adsorbieren und innen fluorapatitähnliche Eigenschaften verleihen (Hellwig 1996). Bei beginnender Neutralisation kann in Anwesenheit von Fluorid Fluorapatit aufgrund seiner geringeren Löslichkeit schneller ausfallen als Hydroxylapatit, sodass die Demineralisationswirkung auf den Schmelz schon bei niedrigerem $\mathrm{pH}$ - Wert reduziert wird. Es kommt zu einer Verkürzung der Demineralisationsperioden, da früher wieder Mineralien präzipitieren. Zugleich wird durch Erhöhung des Fluorapatitanteils an der Zahnoberfläche die Resistenz gegenüber nachfolgenden kariösen Attacken erhöht (Fischer et al. 1994, Hellwig 1996).

Der menschliche Speichel stellt in der Regel eine mit Kalzium übersättigte Lösung dar. Durch die Anwendung topischer Fluoride kommt es zur Ausfällung von Kalziumfluorid, das einen stabilen Niederschlag auf der Zahnoberfläche bildet (ten Cate und Featherstone 1991). Dieses Präzipitat bildet beim kariösen Angriff ein Reservoir freier Fluoridionen, indem diese bei einem $\mathrm{pH}$ - Wert- Abfall aus der Kalziumfluoridschicht freigesetzt werden (Fischer et al. 1994). 


\section{Beeinflussung des Bakterienmetabolismus}

Wenn organische Säuren durch die kariogenen Bakterien gebildet werden, kommt es zu einem $\mathrm{pH}$ - Wert- Abfall, einer Verbindung von im Speichel vorhandenen Wasserstoff- und Fluoridionen und somit zu einem Anstieg von Fluorwasserstoff. Dieser kann im Gegensatz zu ungebundenen Fluoridionen in die Bakterienzellen eindringen (Fejerskov et al. 1996). In der Zelle dissoziiert der Fluorwasserstoff wieder und die freigesetzten Fluoridionen hemmen für den Bakterienmetabolismus wichtige Enzymaktivitäten (Van Loveren 1990, Fejerskov et al. 1996). Des Weiteren führt die Zunahme der Wasserstoffionen zu einem intrazellulären $\mathrm{pH}-$ Wert- Abfall. Da die Enzyme der Glykolyse ein pH- Optimum im alkalischen Bereich besitzen, wird auch auf diese Weise die Enzymaktivität gehemmt (Hellwig et al. 2009). Es resultiert eine Hemmung des Membrantransports von Glucose, eine Glykolysehemmung sowie eine Hemmung der intrazellulären Polysaccharidsynthese (Featherstone 2000, Hellwig et al. 2009).

Eingesetzt in therapeutischen Dosen ist Fluorid für den menschlichen Organismus unschädlich (Hellwig 1996). Jedoch kann es im Zuge einer latenten toxischen Fluoridzufuhr bis zum achten Lebensjahr zu Fluorosen kommen (Limeback 1994, Ismail und Hasson 2008, Denbesten und Li 2011). Des Weiteren können Reizungen der Magenschleimhaut auftreten, wenn Fluoride in höheren Konzentrationen verschluckt werden. Folglich sollten im Zuge von Fluoridierungsmaßnahmen evidenzbasierte Empfehlungen befolgt und die korrekte Anwendung der Fluoridierungsprodukte sichergestellt werden (Hellwig 1996).

\subsubsection{Chlorhexidin}

Chlorhexidindigluconat ist gekennzeichnet durch sein breites, unspezifisches Wirkungsspektrum, seine lange Wirkdauer sowie durch seine große Bandbreite an Einsatzmöglichkeiten, weshalb es unter den oralen Antiseptika nach wie vor den Goldstandard darstellt (Ruppert und Schlagenhauf 2004). Es handelt sich um ein in Wasser oder Alkohol lösliches Digluconatsalz, das über eine geringe Oberflächenspannung verfügt und durch reversible elektrostatische Bindungen an Zahnhartsubstanzen, Schleimhäuten und Mikroorganismen adsorbiert (Davies 1973, Hjeljord et al. 1973). Dabei besteht eine Dosis- Wirkungsbeziehung (Loe und Schiott 1970, Bonesvoll et al. 1974, Jenkins et al. 1994). Eingesetzt in niedrigen Konzentrationen wirkt es bakteriostatisch, indem durch Anlagerung der kationischen $\mathrm{CHX}$ - Moleküle an die Zellwand und orale Glykoproteine der interzelluläre Membrantransport der Mikroorganis- 
men und die Adhärenz der Bakterien gestört werden. In höheren Konzentrationen penetriert der Wirkstoff die Bakterienzellwand und es kommt zur Präzipitation des Zytoplasmas, sodass eine primär bakterizide Wirksamkeit besteht.

Die systemische Toxizität ist gering, und es kommt nur selten zu Überempfindlichkeitsreaktionen; jedoch können bei längerfristiger Anwendung Nebenwirkungen wie zum Beispiel die reversible Verfärbung von Zähnen und Zunge auftreten (Schiiott et al. 1976, Arweiler et al. 2011).

\subsubsection{Möglichkeiten der Anwendung}

In zahlreichen Studien wurde die Effektivität verschiedener Fluorid- und $\mathrm{CHX}$ - Präparate in der Prävention von White- Spot- Formationen untersucht. Aufgrund einer Vielzahl an Anwendungsmöglichkeiten wird kontrovers diskutiert, welche Form der Anwendung die optimale Präventionsstrategie im Rahmen der Behandlung mit festsitzenden Apparaturen darstellt.

\section{Fluoridanwendungen}

Die lokale Fluoridapplikation kann in Form von Zahnpasten, Mundspüllösungen, Gelen und Lacken erfolgen, die Konzentration, Anwendungshäufigkeit und das Dosierungsschema variieren dabei je nach angewendeter Applikationsform (DGZMK 2007). Fluoridhaltige Zahnpasta hat maßgeblich Einfluss auf den verzeichneten Kariesrückgang der letzten Jahre genommen, stellt noch heute die verbreiteste Form der Fluoridanwendung dar und sollte bei jedem Patienten die Grundlage des Fluoridregimes bilden (König 1993, DGZMK 2007). Der zusätzliche Einsatz fluoridhaltiger Mundspüllösungen kann das Demineralisationsrisiko während der kieferorthopädischen Behandlung effektiv reduzieren (Benson et al. 2004, Ogaard et al. 2006). Geiger et al. (1992) zeigten, dass durch tägliche Verwendung einer 0,05 prozentigen Natriumfluoridmundspüllösung ein signifikant geringeres Auftreten von White- Spot- Läsionen erzielt werden konnte. Jedoch wurde ebenso in der Studie deutlich, dass die Effektivität dieser Fluoridierungsmaßnahme maßgeblich von der Mitarbeit des Patienten abhängig ist. Eine weitere Möglichkeit der lokalen Fluoridierung im Rahmen der Multibandbehandlung besteht in der Verwendung fluoridhaltiger Gele (Alexander und Ripa 2000, Splieth et al. 2011). So kann die einmal wöchentliche Anwendung von Elmex gelée ${ }^{\circledR}$ dem Kariesanstieg bei Patienten mit festsitzenden Apparaturen entgegenwirken (Denes und Gabris 1991). Im Gegensatz zu den zuvor genannten Anwendungsmöglichkeiten erfolgt die Applikation von fluoridhaltigen Lacken ausschließlich durch das zahnärztliche Behandlungsteam und ist daher nicht von der Compliance des Patienten abhängig. Weitere Vorteile der Fluoridlacke bestehen in der ge- 
zielten und einfachen Applikationsmöglichkeit und der prolongierten Fluoridfreisetzung im Sinne eines „slow release“, wodurch der Lack weniger oft appliziert werden muss als Gele und Mundspüllösungen (Petersson et al. 2004, Kneist et al. 2008). Fluoridlacke sollten bei Patienten mit erhöhtem Kariesrisiko in einem Abstand von drei bis sechs Monaten appliziert werden und können zusätzlich zu anderen Fluoridierungsmaßnahmen eingesetzt werden (Petersson 1993, DGZMK 2007).

Fluoridfreisetzende Befestigungsmaterialien von Brackets und Bändern, fluoridfreisetzende Versiegelungslacke (Sealer) oder Elastics stellen eine weitere von der Compliance unabhängige Möglichkeit der White- Spot- Prävention dar (Benson et al. 2004). Zur Bracketbefestigung können fluoridhaltige Kompositadhäsive oder fluoridfreisetzende Zemente eingesetzt werden. Der potentielle Benefit dieser Materialien besteht in der konstanten Freisetzung von Fluoriden über einen längeren Zeitraum (Cohen et al. 2003). Glasionomerzemente weisen dabei die höchste Freisetzung von Fluoriden sowie die höchste Kapazität der Fluoridwiederaufnahme auf (Cohen et al. 2003, Rao und Malhotra 2011). Fluoridfreisetzende Versiegelungslacke können vor oder nach dem Kleben der Brackets auf die Schmelzoberfläche appliziert werden, um versehentlich angeätzten Schmelz zu versiegeln, die Haftkraft des Adhäsivs $\mathrm{zu}$ steigern und eine Demineralisation des Bracketumfeldes zu verhindern $\mathrm{Hu}$ und Featherstone 2005). Nicht, beziehungsweise wenig gefüllte Sealer weisen eine geringe Abrasionsresistenz auf, sodass die Sealerschicht mit der Zeit durch abrasive Vorgänge wie das Zähneputzen reduziert wird und somit kein Schutz vor Demineralisation mehr besteht (Benham et al. 2009). Um eine größere Abrasionsresistenz und damit einen dauerhaften Demineralisationsschutz zu gewährleisten, wurden lichthärtende Sealer mit höherem Füllstoffgehalt entwickelt.

\section{Chlorhexidinanwendungen}

$\mathrm{CHX}$ kann in Form von Mundspüllösungen, Gelen oder Lacken angewendet werden, um die Keimzahlen kariogener Bakterien zu senken (Kneist et al. 2008). Die nachhaltigste Reduktion an Streptococcus mutans wird dabei durch CHX- Lacke, gefolgt von Gelen, und letztlich Mundspüllösungen erreicht (Emilson 1994).

Brightman et al. (1991) erzielten durch die Verwendung einer 0,12\%igen CHX- Mundspüllösung eine signifikante Plaque- und Gingivitisreduktion bei Patienten mit festsitzenden Apparaturen. In Studien von Madlena et al. (2000) und Twetman et al. (1995) wurde in splitmouth- Modellen die Effektivität des chlorhexidinhaltigen Lackes Cervitec ${ }^{\circledR}$ untersucht. Bei Madlena et al. konnte die Anzahl neuer Kariesläsionen auf der Testseite durch den Lack signifikant reduziert werden. Demgegenüber konnten Twetman et al. zwar eine signifikante Re- 
duktion der Anzahl an S. mutans feststellen, die Inzidenz initialkariöser Läsionen war jedoch generell gering, und es ließen sich diesbezüglich keine signifikanten Unterschiede zwischen Test- und Kontrollseite feststellen.

Aufgrund fehlender Evidenz wird der Einsatz von CHX im Rahmen der WSL- Prophylaxe während kieferorthopädischer Behandlungen sehr unterschiedlich bewertet. Attin et al. (2005) zeigten, dass die Effektivität von CHX durch die Anwesenheit von Brackets und Bändern negativ beeinflusst wird, sahen dennoch die antimikrobielle Therapie als „einzig versprechende Alternative zu verbesserter Mundhygiene, Fluoridierungsmaßnahmen und Ernährungsberatung in der Kariesprävention“ (Attin et al. 2005, S.492).

\subsection{Studienlage zur Erzeugung von Demineralisationen in vitro}

In- vitro- Studienmodelle stellen nach wie vor die meist verwendeten Methoden in der Kariesforschung dar (White 1995, Buzalaf et al. 2010). Die Pathogenese kariöser Läsionen kann unter definierten, reproduzierbaren und kontrollierten Bedingungen untersucht werden, es können gezielt einzelne Einflussfaktoren auf die Pathogenese und Prävention eruiert und ethische Problematik vermieden werden (Sissons 1997, Seemann et al. 2005).

Bei der Erzeugung von Demineralisationen in vitro kann grundsätzlich zwischen chemischen und biologischen Systemen unterschieden werden (Fontana et al. 1996).

\subsubsection{Chemische Systeme}

Die chemische Erzeugung von Demineralisationen erfolgt durch direkte Einwirkung von Säuren, die in Form säurehaltiger Medien wie Gelen oder Lösungen eingesetzt werden (Featherstone und Mellberg 1981). Bei den verwendeten Säuren handelt es sich vorwiegend um Essig- oder Milchsäure. 1982 etablierten ten Cate und Duijsters ein $\mathrm{pH}$-cycling-Modell, in dem Lösungen mit unterschiedlichem $\mathrm{pH}$ - Wert täglich mehrfach alternierend eingesetzt wurden, um den intraoralen Wechsel von De- und Remineralisationsphasen beim Menschen zu simulieren. Die Vorteile chemischer Systeme bestehen in der relativ einfachen Handhabung, der Kontrollierbarkeit der experimentellen Bedingungen, der kostengünstigen Durchführung und der Reproduzierbarkeit (Fontana et al. 1996). Jedoch unterscheiden sich die erzeugten Läsionen von den durch Mikroorganismen hervorgerufenen Demineralisationserscheinungen, da durch Anwendung chemischer Systeme letztlich nur Lösungsvorgänge voll- 
zogen werden und eine bakterielle, proteolytische Proteindestruktion unterbleibt. Darüber hinaus kann ohne Anwesenheit von Mikroorganismen der kariesinhibierende Effekt antimikrobieller Wirkstoffe nicht getestet werden (Schiffner und Schmeiser 1995).

\subsubsection{Biologische Kariesmodelle}

Bei der Verwendung biologischer Kariesmodelle wird der Demineralisationsprozess durch Mikroorganismen hervorgerufen. Zumeist wird S. mutans in den biologischen Modellen eingesetzt. Es existieren einfache Kariesmodelle, bei denen vorkultivierte Bakterien in ein Nährmedium inokuliert werden. In dieser Bakteriensuspension werden dann Prüfkörper im weiteren Studienverlauf der Demineralisation durch die Bakterien ausgesetzt (Dummer et al. 1982). Die einfachen Kariesmodelle kennzeichnen sich dadurch, dass es zu keiner komplexen Biofilmbildung kommt, da zumeist nur Mono- oder Dikulturen in einer einfachen Versuchsanordnung verwendet werden und somit keine komplexe Plaquestruktur mit genetischer oder räumlicher Heterogenität aufgebaut wird (Sissons 1997).

Das Bestreben, die intraorale Situation möglichst naturgetreu zu simulieren, führte zur Entwicklung komplexerer Kariesmodelle (Deng und ten Cate 2004, Seemann et al. 2005, Hayati et al. 2011). Es wurden künstliche Mundhöhlen entwickelt, die sich in den letzten Jahrzehnten von simplen Apparaturen zu hoch komplexen, computerkontrollierten Versuchsmodellen entwickelt haben. Der grundlegende Aufbau einer künstlichen Mundhöhle besteht aus einer Apparatur, die durch kontinuierliche oder intermittierende Zufuhr von Nährstoffen und Simulation des intraoralen Milieus das Wachstum eines Biofilms ermöglicht. Die Bakterien können in Rein- oder Mischkultur eingesetzt werden. Während mit den einfachen Modellen vorwiegend Untersuchungen der Ätiologiefaktoren der Karies studiert wurden, können mit den heutigen künstlichen Mundhöhlen das Wachstum, die Pathogenität, der Metabolismus und die mikrobiellen Interaktionen dentaler Plaque unter hochkontrollierten Bedingungen untersucht werden. Durch ihren komplexen Aufbau mit oftmals mehreren Reaktionskammern und verschiedenen Regulationsmechanismen, wie Temperatur-, Gas- und pH- Kontrolle sowie kontrollierter Zufuhrmöglichkeit verschiedener Medien, kann ein Milieu geschaffen werden, das die intraoralen Gegebenheiten möglichst detailgenau simuliert (Tang et al. 2003). 


\subsection{Demineralisationsdiagnostik in vitro}

\subsubsection{Die quantitative lichtinduzierte Fluoreszenz}

Die quantitative lichtinduzierte Fluoreszenz (QLF) stellt eine nicht- invasive Methode zur Kariesdiagnostik dar, deren Funktionsprinzip auf den autofluoreszierenden Eigenschaften der Zahnhartgewebe beruht (Ifland et al. 2004). Durch Bestrahlung mit Licht des blaugrünen Spektralbereiches wird eine Fluoreszenz der Zahnhartsubstanzen induziert. Das vom Zahn zurückgestrahlte Licht wird durch eine digitale Kamera mit Gelbfilter aufgenommen, als Fluoreszenzbild auf dem PC wiedergegeben und kann mit einer speziellen Software analysiert werden (Abb.2.3). Die Fluoreszenzintensität ist dabei mit dem Mineralgehalt der Zahnhartgewebe korreliert. Eine Demineralisation der Zahnhartgewebe führt zu einer Verringerung des Mineralgehaltes und damit der Autofluoreszenz (Tranaeus et al. 2005). Dieser Unterschied der Fluoreszenzintensität ermöglicht die Quantifizierung der Demineralisation mit Hilfe der QLF- Software (van der Veen und de Josselin de Jong 2000). Die bedeutendsten von der Software bestimmten Parameter sind das Läsionsareal (WS Area in $\mathrm{mm}^{2}$ ), die Läsionstiefe ausgedrückt durch die prozentuale Fluoreszenzabnahme $(\Delta \mathrm{F}$ in \%) und das Läsionsvolumen $\left(\Delta \mathrm{Q}\right.$ in $\mathrm{mm}^{2} \%$ ) (Inspektor Research Systems BV 2000).

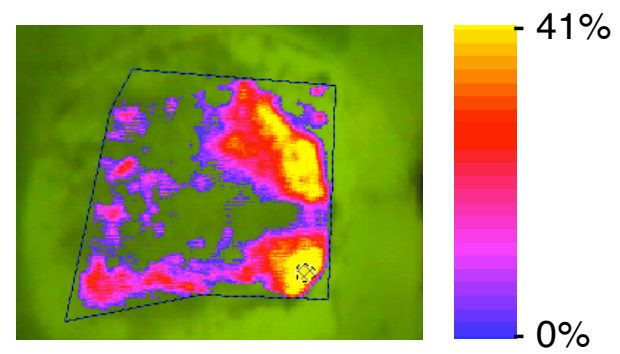

Abb.2.3 Auswertungsbereich eines Prüfkörpers nach der QLF- Analyse; die Farbskala gibt die prozentuale Fluoreszenzabnahme wieder

\subsubsection{Weitere Verfahren}

\section{Polarisationsmikroskopie}

Die Polarisationsmikroskopie ermöglicht die Erfassung qualitativer Veränderungen des Mineralgehaltes, Läsionscharakteristika sowie quantitative Analysen des Porenvolumens von Schmelzproben (Arends und ten Bosch 1992). Bei einem Polarisationsmikroskop wird durch einen Polarisationsfilter (Polarisator) unterhalb des Objekttisches nur polarisiertes Licht durchgelassen. Oberhalb des Objekttisches befindet sich ein weiterer Filter (Analysator), der 
einen Durchtritt des polarisierten Lichtes vollständig verhindert. Befindet sich auf dem Objekttisch jedoch ein anisotroper Körper, bewirkt dieser aufgrund seiner Eigendoppelbrechung eine Änderung der Polarisationsebene des Lichtes, ein Teil der Lichtstrahlen kann den Analysator passieren und entsprechende Strukturen werden sichtbar (Wenisch 2009).

\section{Mikrohärtemessung}

Bei diesem Härtemessverfahren wird ein Diamantkörper mit definierter Prüfkraft für eine bestimmte Zeit auf den zu testenden Prüfkörper gedrückt. Die Größe der resultierenden permanenten Eindellung wird mit einem Mikroskop untersucht, vermessen und zum Mineralgehalt des ausgesetzten Prüfkörpers in Relation gesetzt. Bei einer Zunahme der Eindrücktiefe besteht ein Mineralverlust im Gewebe (Ten Bosch und Angmar-Mansson 1991). Das Verfahren ermöglicht jedoch lediglich qualitative Aussagen über Mineralverlust oder -gewinn (Arends und ten Bosch 1992).

\section{Transversale Mikroradiographie}

Bei der transversalen Mikroradiographie (TMR) werden die Prüfkörper in dünne, planparallele und senkrecht zur anatomischen Zahnoberfläche ausgerichtete Scheiben geschnitten. Das weitere Vorgehen besteht darin, die Schnittpräparate zusammen mit einer Aluminiumeichtreppe auf einem Röntgenfilm zu fixieren und einer monochromatischen Röntgenstrahlung auszusetzen. Mit Hilfe der ebenfalls abgebildeten Aluminiumeichtreppe kann bei bekannter Dicke des Schliffpräparates der Mineralgehalt in Abhängigkeit zur Läsionstiefe bestimmt werden. Die Absorption der Röntgenstrahlen wird durch die optische Dichte des entwickelten Filmes wiedergegeben und mit Hilfe der Dosimetrie kann der Mineralgehalt bestimmt werden. Die transversale Mikroradiographie stellt das am häufigsten angewendete Verfahren in der in- vitro- Kariesdiagnostik dar und gilt als Goldstandard zur Bestimmung des Mineralgehaltes von Zahnhartgeweben (Arends und ten Bosch 1992, Gmur et al. 2006).

\section{Confokale Laser Scanning Mikroskopie (CLSM)}

Bei dem nicht- destruktiven Verfahren der CLSM wird das zu untersuchende Objekt Punkt für Punkt mit einem Laserstrahl abgetastet, und das aus der jeweiligen Fokusebene reflektierte Licht wird detektiert. Durch die einzelnen Detektionen wird eine tomogrammähnliche Bildserie erstellt, die anschließend mit Hilfe eines Rechners zu einem vollständigen dreidimensionalen Bild zusammengefügt werden kann (Zentner und Duschner 1996). 


\section{Versuchsplanung}

\section{Aufbau des verwendeten Kariesmodells}

In Rahmen der Studie wurde ein biologisches Kariesmodell angewendet, in dem eine Bakteriensuspension als Demineralisationslösung eingesetzt wurde. Dazu wurde ein auf Blutagarplatten vorkultivierter S. mutans- Stamm in eine Bouillon mit Glucose inokuliert. Des Weiteren wurde eine Remineralisationslösung in Form von künstlichem Speichel eingesetzt.

Um das menschliche Ernährungsverhalten im Tagesverlauf und damit den stetigen Wechsel des intraoralen $\mathrm{pH}$ - Wertes zu simulieren, wurden die Prüfkörper an jedem Versuchstag stundenweise alternierend der De- und Remineralisationslösung ausgesetzt, analog dem Wechsel von Nahrungsaufnahme und substratfreien Intervallen zwischen den Mahlzeiten beim Menschen. Zur Kontrolle, dass es nur unter Anwesenheit von kariogenen Bakterien zum Auftreten kariöser Läsionen kommt, wurde eine Negativkontrollgruppe gebildet, deren Prüfkörper während der Demineralisationsphasen einer Glucose- Bouillon ohne S. mutans ausgesetzt waren.

\section{Versuchsablauf}

Zunächst erfolgte die Herstellung von 65 Prüfkörpern. Diese wurden anschließend fünf Versuchsgruppen (A Cervitec Plus ${ }^{\circledR}$, B ProSeal ${ }^{\circledR}$, C Maximum Cure ${ }^{\circledR}$, D Positivkontrolle und E Negativkontrolle) randomisiert zugewiesen. Vor Beginn der Versuchsphase erfolgte die Baselinemessung mittels quantitativer lichtinduzierter Fluoreszenz (QLF).

Die eigentliche Versuchsphase umfasste einen Zeitraum von 30 Tagen. Am ersten Versuchstag wurden die getesteten Produkte nach Herstellerangaben appliziert. Bei den Gruppen $D$ und E erfolgten keine Produktapplikationen. Anschließend begann der Zyklus des ersten Versuchstages: Zunächst wurden die Prüfkörper der jeweiligen Gruppen für eine Stunde in der Remineralisationslösung gelagert. Anschließend folgten einstündige, alternierende Deund Remineralisationszyklen mit abwechselnder Lagerung in den entsprechenden Lösungen. Am Ende des Versuchstages wurden alle Prüfkörper mit destilliertem Wasser abgespült und über Nacht in künstlichem Speichel gelagert. Dieser Versuchszyklus wurde an allen $30 \mathrm{Ta}-$ gen der Versuchsphase wiederholt. An den Versuchstagen $\mathrm{t} 1=7, \mathrm{t} 2=14$ und $\mathrm{t} 3=30$ wurden Messungen mit der QLF durchgeführt. In Abbildung 3.1 ist in einem Fließdiagramm eine Übersicht des Versuchsablaufes dargestellt. 


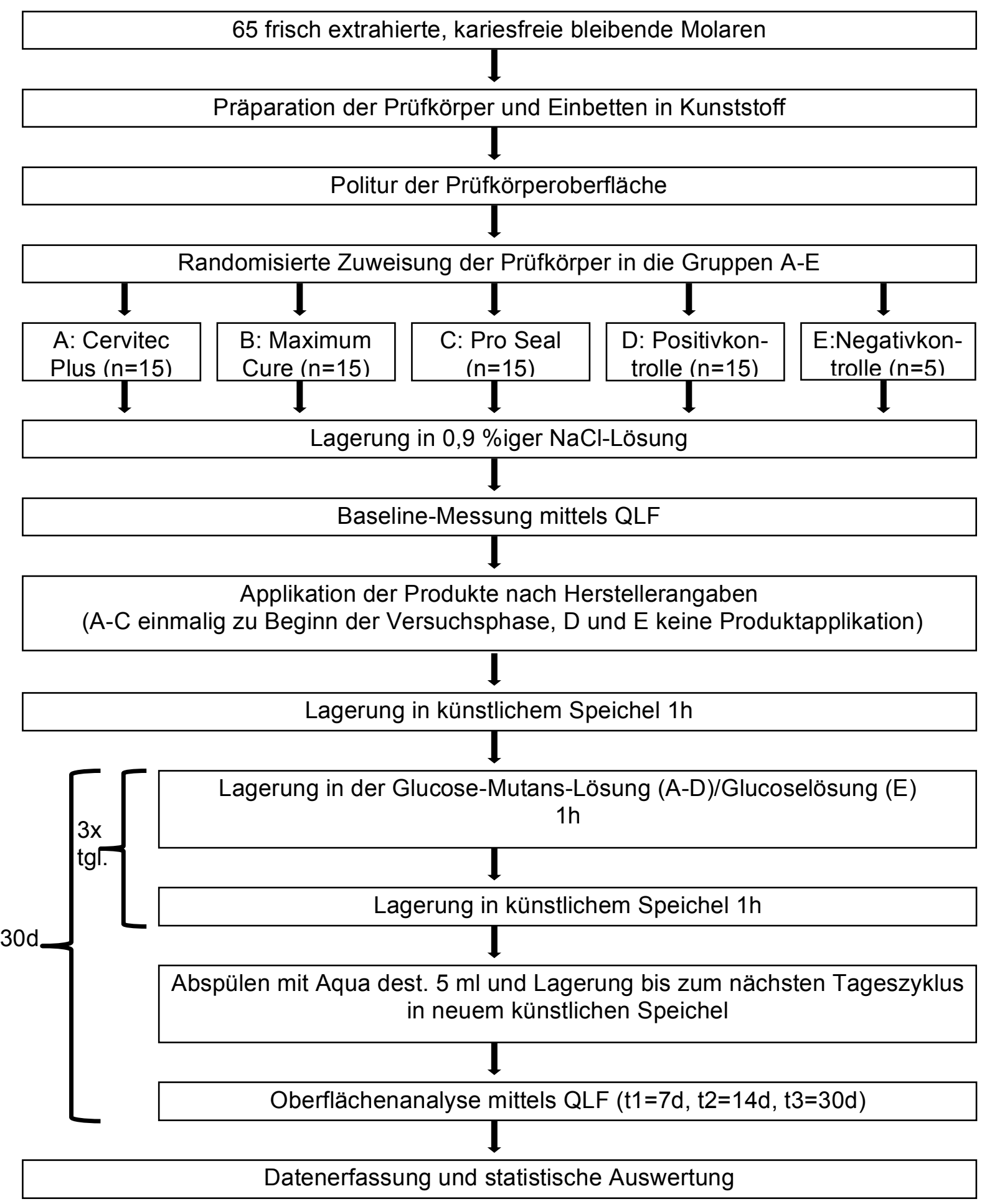

Abb.3.1 Übersicht über den Versuchsablauf 


\section{Material und Methode}

\subsection{Materialien}

\subsubsection{Zähne}

Für die Herstellung der Schmelzprüfkörper wurden 65 frisch extrahierte, menschliche, kariesund füllungsfreie Molaren der zweiten Dentition verwendet.

Diese wurden nach der Extraktion mit Hilfe eines scharfen Skalpells und destilliertem Wasser von Blut- und Geweberesten befreit und bis zur Probenherstellung in 0,9 prozentiger $\mathrm{NaCl}$ Spüllösung (B.Braun Melsungen AG, Melsungen, Deutschland) bei Raumtemperatur gelagert. In regelmäßigen Abständen erfolgte ein Wechsel der $\mathrm{NaCl}$ - Spüllösung, um ein Bakterienwachstum zu vermeiden.

\subsubsection{Bakterien}

Für das Kariesmodell wurde ein kariogener Stamm von Streptococcus mutans (Clarke 1924, DSM 20523, ATCC 25175) in Reinkultur eingesetzt. Dieser wurde von der Deutschen Sammlung von Mikroorganismen und Zellkulturen $\mathrm{GmbH}$ (Braunschweig, Deutschland) bezogen. Die Vorkultivierung der Bakterien erfolgte auf Blut- Agar- Platten (Columbia- Agar mit 5\% Schafsblut (COS), bioMérieux $\mathrm{GmbH}$, Nürtingen, Deutschland).

\subsubsection{Medien}

\subsubsection{Demineralisationslösung}

Als flüssiger Nährboden wurde eine Bouillon mit Glucose mit einem pH- Wert von 6,5-7 verwendet, in die die vorkultivierten Bakterien überimpft wurden.

Zunächst wurde die Bouillon nach Rezeptur des Instituts für Medizinische Mikrobiologie (Zentrum Hygiene und Humangenetik, Universitätsmedizin Göttingen, Deutschland) hergestellt. Die genaue Zusammensetzung ist in Tabelle 4.1 aufgelistet. 
Tab.4.1 Bouillon Zusammensetzung (10 Liter)

\begin{tabular}{|l|r|}
\hline Bestandteil & Menge \\
\hline $\mathrm{NaCl}$ (Merck 6404) & $50 \mathrm{~g}$ \\
\hline Pepton aus Fleisch (Merck 7214) & $100 \mathrm{~g}$ \\
\hline Aqua dest. & Ad 10 Liter \\
\hline $\mathrm{NaOH}$ & $6 \mathrm{ml}$ \\
\hline
\end{tabular}

In einem zweiten Schritt wurde der fertigen Bouillon Glucose (10g Glucose auf 1000ml BouilIon) hinzugefügt. Abschließend wurde die Bouillon mit Glucose im Autoklaven für 20 Minuten bei $120^{\circ} \mathrm{C}$ sterilisiert. Die Lagerung der fertigen Bouillon mit Glucose erfolgte im Kühlschrank bei $4^{\circ} \mathrm{C}$.

\subsubsection{Remineralisationslösung}

Als Remineralisationslösung wurde künstlicher Speichel NRF 7,5 verwendet, der von der Zentralapotheke der Universitätsmedizin Göttingen bezogen wurde. Die genaue Zusammensetzung ist der Tabelle 4.2 zu entnehmen.

Tab.4.2 Zusammensetzung künstlicher Speichel

\begin{tabular}{|l|r|}
\hline Bestandteil & 50g Lösung enthalten \\
\hline Sorbit & $1,5050 \mathrm{~g}$ \\
\hline $\mathrm{KCl}$ & $0,0600 \mathrm{~g}$ \\
\hline $\mathrm{NaCl}$ & $0,0425 \mathrm{~g}$ \\
\hline Magnesiumchlorid $\times 6 \mathrm{H}_{2} \mathrm{O}$ & $0,0025 \mathrm{~g}$ \\
\hline Calciumchlorid $\times 2 \mathrm{H}_{2} \mathrm{O}$ & $0,0075 \mathrm{~g}$ \\
\hline di-Na-monohydrogenphosphat $\times 12 \mathrm{H}_{2} \mathrm{O}$ & $0,1250 \mathrm{~g}$ \\
\hline Carmellose-Natrium (7 MF PH) & $0,2500 \mathrm{~g}$ \\
\hline Gereinigtes Wasser & Ad 50,0000g \\
\hline Konserviert mit: 0,1\% Sorbinsäure & \\
\hline
\end{tabular}

\subsubsection{Verwendete Testprodukte}

Im Rahmen der Studie wurden der Lack Cervitec Plus ${ }^{\circledR}$ (Ivoclar Vivadent AG, Schaan, Lichtenstein) sowie die Versiegelungslacke Pro Seal ${ }^{\circledR}$ (Reliance Orthodontic Products, Itasca, IL, 
USA) und Maximum Cure ${ }^{\circledR}$ (Reliance Orthodontic Products, Itasca, IL, USA) verwendet. Die Produkte unterscheiden sich hinsichtlich ihrer Zusammensetzung, ihres kariesprophylaktischen Wirkstoffes beziehungsweise dessen Konzentration und ihres Applikationsverfahrens. Im Folgenden werden die Produkte kurz vorgestellt:

\section{Cervitec Plus ${ }^{\circledR}$ (Ivoclar Vivadent AG, Schaan, Lichtenstein)}

Die kariesprophylaktische Wirksamkeit des Lackes beruht nach Angaben des Herstellers auf seinem Gehalt an Chlorhexidin (1\%) und Thymol (1\%), Fluorid ist nicht enthalten. Thymol gehört zur Gruppe der Phenole und ist Bestandteil eines ätherischen Öles.

\section{MaximumCure ${ }^{\circledR}$ (Reliance Orthodontic Products, Itasca, IL, USA)}

Maximum Cure ${ }^{\circledR}$ ist ein chemisch härtender, ungefüllter Versiegelungslack und ist durch einen Fluoridgehalt von 2-4 Gewichtsprozent gekennzeichnet.

\section{Pro Seal® (Reliance Orthodontic Products, Itasca, IL, USA)}

Bei Pro Seal handelt es sich um einen lichthärtenden Versiegelungslack, der seine kariesprophylaktische Wirksamkeit durch seinen Fluoridgehalt (5-40 Gew\%) erzeugen soll. Der Lack verfügt über einen hohen Füllstoffanteil.

Der Tabelle 4.3 ist die genaue Zusammensetzung der einzelnen Testprodukte zu entnehmen.

Tab.4.3 Zusammensetzung der getesteten Produkte

\begin{tabular}{|l|l|r|}
\hline Produkt & Inhaltsstoffe & Gew.\% \\
\hline Cervitec Plus ${ }^{\circledR}$ & Ethanol, Wasser & 90 \\
& Vinylacetat Co-Polymer, Acrylat Co-Polymer & 8 \\
& Thymol & 1 \\
& Chlorhexidin- Diacetat & 1 \\
\hline Pro Seal® & Ethoxyliertes Bisphenol- A- Diacrylat & $10-50$ \\
(1 Komponentensystem) & Urethanacrylatester & $10-40$ \\
lichthärtend & Polyethylengylkoldiacrylat & $10-40$ \\
& Fluoridhaltige Glasmasse & $5-40$ \\
\hline Maximum Cure ${ }^{\circledR}$ & Komponente1: & \\
(2 Komponentensystem $)$ & Bisphenol-A-diglycidmethacrylat & $50-80$ \\
chemisch-härtend & Methylmethacrylat & $20-40$ \\
& Hydrofluoridmethacrylat & $2-5$ \\
\cline { 2 - 3 } & Komponente 2: & $50-80$ \\
& Bisphenol-A-diglycidylmethacrylat & $1-5$ \\
& Benzoylperoxid & $20-40$ \\
\hline
\end{tabular}




\subsection{Methode}

\subsubsection{Herstellen der Prüfkörper und Zuweisung in Gruppen}

Ziel der Prüfkörperherstellung war es, 65 Schmelzproben mit einem definierten Durchmesser von $5 \mathrm{~mm}$ zu erhalten.

Um in einem ersten Schritt die Zahnkronen von den Wurzeln zu separieren, wurden die Molaren zunächst in die Halterung der Exakt- Diamantbandsäge (Band System 300/310, EXAKT Advanced Technologies GmbH Norderstedt, Deutschland) eingespannt. AnschlieBend erfolgte bei ausreichender Wasserkühlung mit mittlerer Geschwindigkeit der Bandsäge (Stufe 4,5) und einer Belastung des Schlittens von 70g die Abtrennung der Wurzeln von den Zahnkronen. Danach wurden die Zahnkronen ein weiteres Mal in das Schleifgerät eingespannt, und es erfolgte bei gleicher Wasserkühlung, Geschwindigkeit der Bandsäge und Schlittenbelastung eine Separierung der Zahnkronen in mesio- distaler Richtung, sodass jeweils zwei Kronenhälften gewonnen wurden (Abb. 4.1).
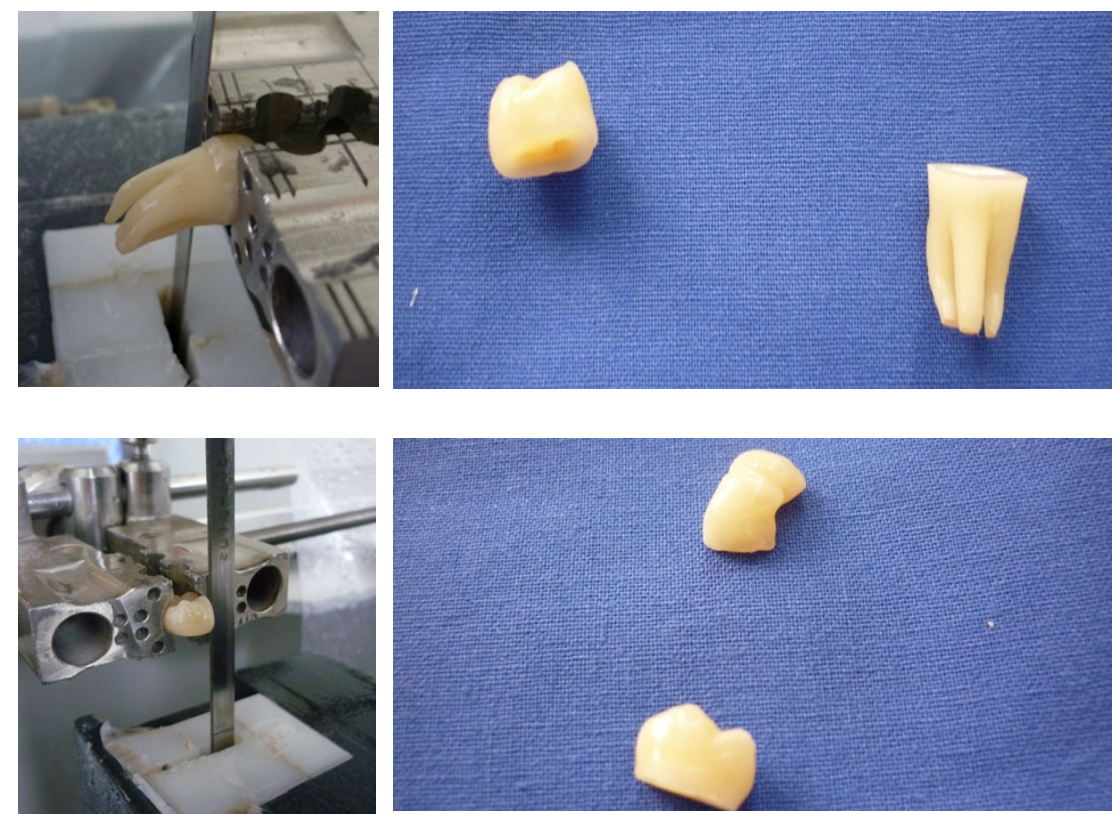

Abb.4.1 Separierung des Zahnes zur Gewinnung von zwei Kronenhälften

Zur Fixierung wurden die Kronenhälften mit Hilfe eines Sekundenklebers (Loktite 420 Sofortklebstoff, Henkel Loctite- KID GmbH, München, Deutschland) und eines chemisch härtenden Kunststoffs (Technovit 4071, Heraeus Kulzer GmbH \& Co. KG, Hanau, Deutschland) auf einer Plexiglasplatte (Objektträger Plexiglas, Patho- Service $\mathrm{GmbH}$, Oststeinbek, Deutsch- 
land) befestigt, wobei die planen Schliffflächen zur Plexiglasplatte wiesen. Nachdem die Plexiglasplatte mit den Kronenhälften in die T.I.P. Tischbohrmaschine (T.I.P. GmbH, Waibstadt, Deutschland) eingespannt wurde, erfolgte bei ausreichender Wasserkühlung mit einem Diamant- Hohlbohrer (10x50 Durchmesser/ Länge, 3,5 Belaghöhe, Dr. Heinemann Diamond Tools \& Parts $\mathrm{GmbH}$, Rastede, Deutschland) das Ausstanzen runder Schmelzproben aus dem Bereich des ehemaligen Zahnäquators mit einem Durchmesser von $5 \mathrm{~mm}$ (Abb.4.2).
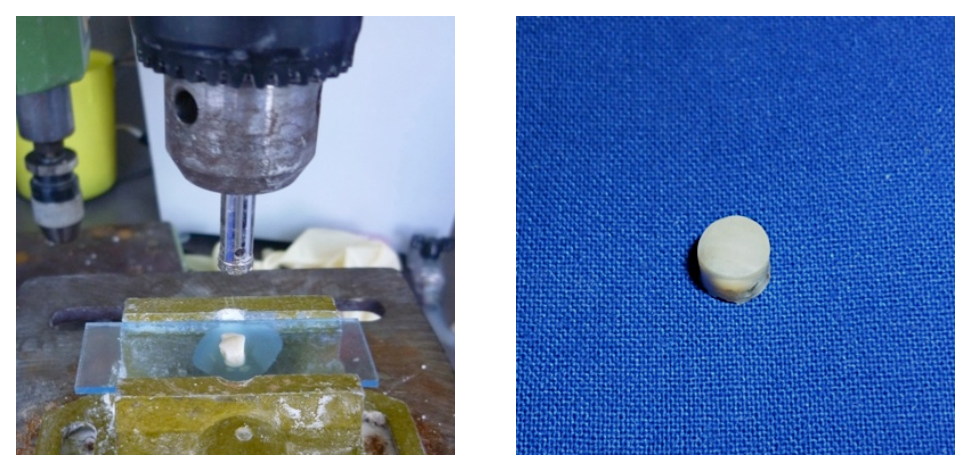

Abb.4.2 Ausstanzen der Kronenhälfte und resultierende Schmelzprobe

Die somit gewonnenen Schmelzproben definierter Größe wurden in einer Silikonform aus Silaplast Futur (DETAX GmbH \& Co. KG, Ettlingen, Deutschland) mit einem chemisch härtenden Kunststoff (Technovit 4071, Heraeus Kulzer GmbH \& Co. KG,Hanau, Deutschland) unter Aussparung der Schmelzoberfläche der Prüfkörper eingebettet (Abb.4.3).

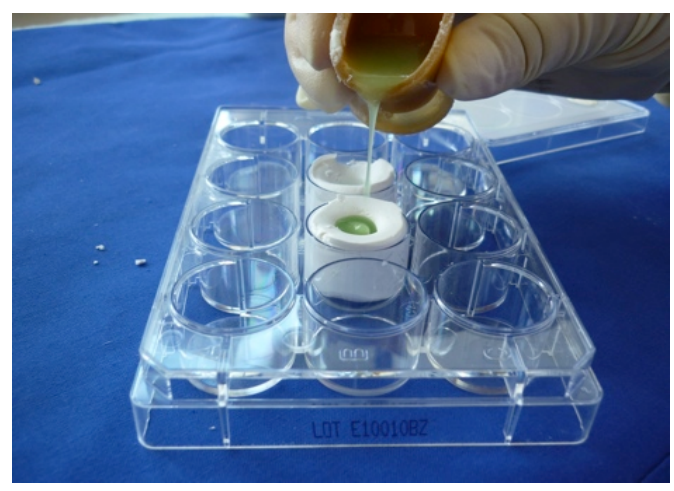

Abb.4.3 Einbetten der Schmelzprobe (die auszusparende Schmelzfläche weist zum Boden der Silaplastform)

Um eine planparallele Oberflächenausrichtung zum Einbettkunststoff zu erreichen und zudem eine gleiche Oberflächenrauigkeit aller Prüfkörper zu gewährleisten, erfolgte eine Politur der Prüfkörperoberflächen mit Hilfe der RotoPol-35 Poliermaschine (STRUERS GmbH, Wil- 
lich, Deutschland) mit einem wassergekühlten Schleifpapier der Körnung P1200 (Hermes) bei 512 Umdrehungen pro Minute (Abb.4.4).

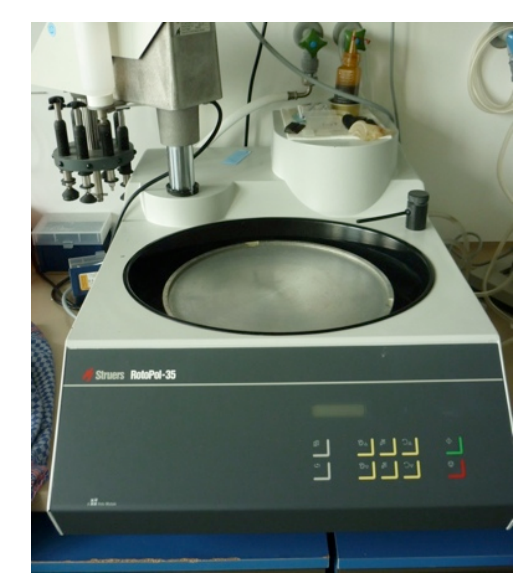

Abb.4.4 RotoPol-35 Poliermaschine

Im Anschluss erfolgte eine Kontrolle der Prüfkörper unter dem Stereomikroskop mit zehnfacher Vergrößerung (Motic, Saarbrücken-Eschringen, Deutschland), um sicherzustellen, dass kein Dentin exponiert wurde und die Schmelzoberfläche intakt war. Ungeeignete Prüfkörper wurden aussortiert. Des Weiteren wurde eine Einkerbung mit einem Winkelstück und einem Diamantzylinder (Komet, Gebr. Brasseler GmbH \& Co KG, Lemgo, Deutschland) unter Wasserkühlung bei $20000 \mathrm{U} / \mathrm{min}$ in die Kunststofffläche aller Prüfkörper präpariert. Die Einkerbung wurde anschließend mit einem wasserfesten Farbstift markiert, um einen definierten Bezugspunkt für die Positionierung unter der QLF- Apparatur sicherzustellen.

Auf diese Weise wurden insgesamt 65 Prüfkörper mit einer Schmelzfläche von 5mm Durchmesser hergestellt (Abb.4.5).

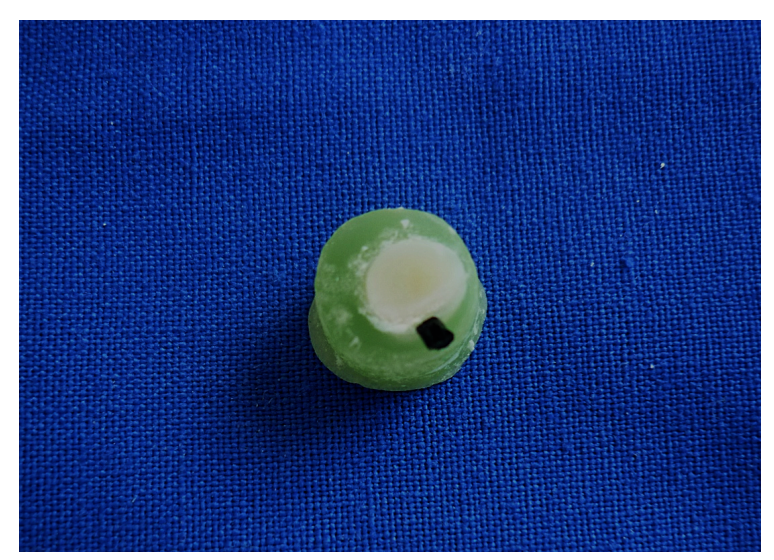

Abb.4.5 Prüfkörper nach der Fertigstellung 
Im Anschluss erfolgte die randomisierte Zuweisung der Prüfkörper in 6 Gruppen:

\begin{tabular}{|c|c|l|}
\hline Gruppe & Prüfkörperanzahl & Testprodukt \\
\hline A & 15 & Cervitec Plus ${ }^{\circledR}$ \\
\hline B & 15 & Maximum Cure ${ }^{\circledR}$ \\
\hline C & 15 & ProSeal ${ }^{\circledR}$ \\
\hline D & 15 & Positivkontrolle \\
\hline E & 5 & Negativkontrolle \\
\hline
\end{tabular}

Abschließend wurde die Rückseite jedes Prüfkörpers mit wasserfestem Farbstift so beschriftet, dass jeder Prüfkörper genau jeder Gruppe und der entsprechenden Datei im QLFSystem eindeutig und reproduzierbar zugeordnet werden konnte. Bis zum Beginn der Versuchsphase wurden die Prüfkörper jeder Gruppe jeweils in einer separaten Schraubdeckeldose $(90 \mathrm{ml}$, 52x53mm, neoLab Migge Laborbedarf- Vertriebs $\mathrm{GmbH}$, Heidelberg, Deutschland), befüllt mit 0,9 prozentiger $\mathrm{NaCl}$ - Spüllösung, gelagert. In regelmäßigen Abständen erfolgte ein Wechsel der $\mathrm{NaCl}$ - Lösung, um ein Bakterienwachstum zu vermeiden.

\subsubsection{Vorkultivierung der Bakterien und Ansetzen der Bakterienlösung}

Für das Kariesmodell wurde als Demineralisationslösung eine Glucose- Bouillon mit einem Streptococcus mutans- Stamm in Reinkultur verwendet. Die Bakterien wurden auf einer BlutAgar- Platte vorkultiviert (Abb.4.6). Die Blut- Agar- Platte wurde in einem Gaspacktopf mit Anearobierpad in einem auf $36,7^{\circ} \mathrm{C}$ temperierten Brutraum gelagert. Da die Bakterienkolonien nach 48 Stunden am ausgeprägtesten waren, wurde dieser Zeitraum für die Vorkultivierung gewählt.

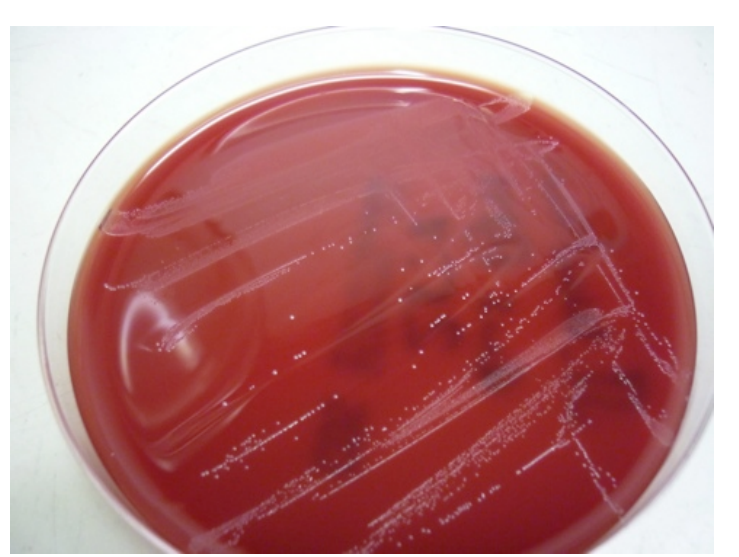

Abb.4.6 S.mutans, kultiviert auf einer Blut- Agar- Platte 
Die Demineralisationslösung wurde täglich für den darauffolgenden Versuchstag hergestellt. Dazu wurden $500 \mathrm{ml}$ der Glucose- Bouillon in einen Erlenmeierkolben gefüllt und anschlieBend 10 Kolonien der vorkultivierten Bakterien überimpft. Der Erlenmeierkolben wurde mit Alufolie und Parafilm (Parafilm „M“, Laboratory film, Pechiney Plastic Packaging, Inc., Chicago, USA) luftdicht verschlossen und bis zum folgenden Versuchstag 23 Stunden in einem Schüttler mit Inkubationshaube (Certomat H., B. Braun Biotech International GmbH, Melsungen, Deutschland) bei $37^{\circ} \mathrm{C}$ und 115 Umdrehungen pro Minute inkubiert (Abb.4,7).

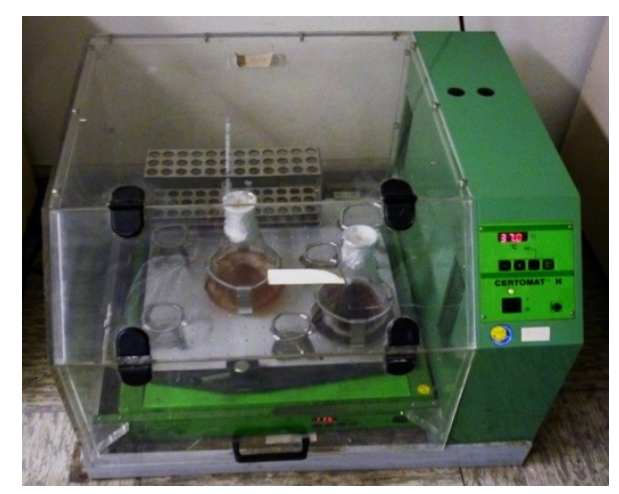

Abb.4.7 Schüttler mit Inkubationshaube

Um eine annähernd gleiche Bakterienkonzentration während der Demineralisationsphasen zu gewährleisten, wurde in einem Vorversuch durch Erstellen einer Wachstumskurve überprüft, in welchem Zeitintervall die optische Dichte der Suspension relativ konstant war. Dazu wurde die Bakteriensuspension gemäß oben genannter Beschreibung angesetzt. AnschlieBend wurde die optische Dichte bei 600nm zu verschiedenen Zeitpunkten mit dem BioPhotometer (Eppendorf AG, Hamburg, Deutschland) gemessen und eine Wachstumskurve erstellt. Die Abbildung 4.8 zeigt die Wachstumskurve der Bakterien in der Demineralisationslösung. Während der Plateauphase, die nach 23 Stunden erreicht wurde, blieb die optische Dichte über mehrere Stunden relativ konstant. Aus diesem Grund wurde dieser Zeitraum (23 - 29 Stunden nach Ansetzen der Lösung) für die Verwendung der Suspension in der Versuchsphase ausgewählt. 


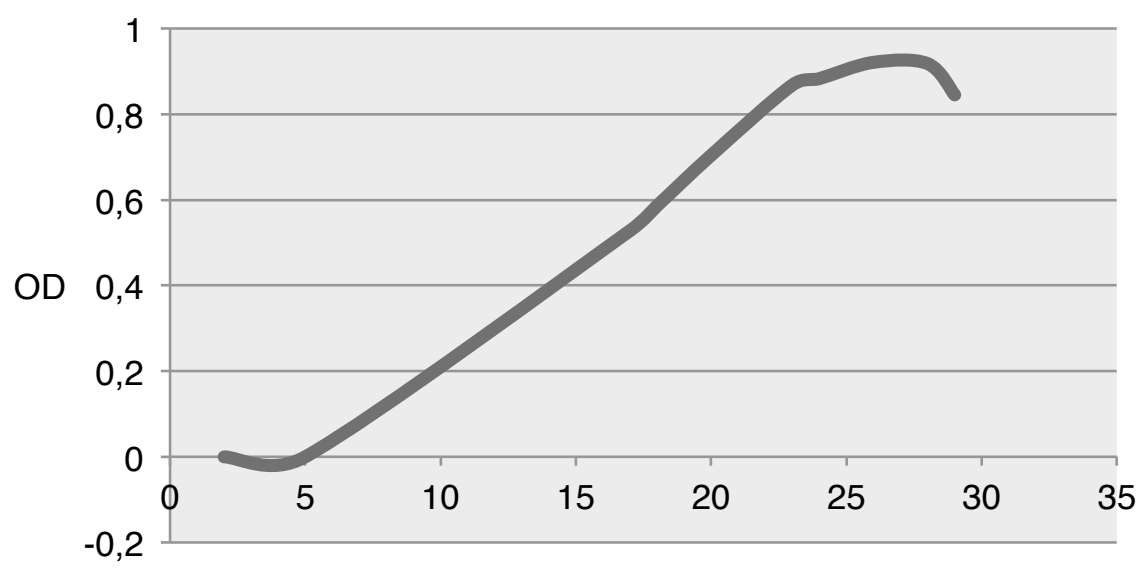

Zeit (in h)

Abb.4.8 Grafische Darstellung der Wachstumskurve: Die mit dem Photometer zu verschiedenen Zeitpunkten gemessene optische Dichte (OD) wurde gegen die Zeit $(\mathrm{h})$ aufgetragen

Um ebenfalls sicherzustellen, dass die Demineralisationslösung auch an allen Versuchstagen eine annähernd identische Konzentration aufweist, wurde täglich unmittelbar vor der ersten Demineralisationsphase die optische Dichte bei 600nm mit Hilfe des BioPhotometers (Eppendorf AG, Hamburg, Deutschland) gemessen. Der Mittelwert der optischen Dichte lag während der gesamten Versuchsphase nach 23 Stunden bei 0,897. Zusätzlich erfolgte eine $\mathrm{pH}$ - Wert- Messung nach 23 Stunden. Der pH- Wert lag bei 4,5. Um zu überprüfen, dass keine unkontrollierte mikrobielle Kontamination vorlag, wurde täglich eine Kontrollprobe der Lösung auf Blut- Agar- Platten ausgestrichen und nach 48 Stunden kontrolliert.

\subsubsection{Messungen und Analyse mittels quantitativer lichtinduzierter Fluoreszenz}

Die Messungen wurden mit der Software Inspektor Pro Version X2.0.0.43 (Inspektor Dental Care BV, Amsterdam, Niederlande) durchgeführt. Mit Hilfe der QLF- Software können live Videobilder angezeigt und Einzelbilder gespeichert werden. Das Zubehör besteht aus einem Objekttisch, einem PC mit eingebauter Netzkarte und dem QLF- Auswertegerät, das mit einer Videokamera mit CCD- Sensor und einer Lichtquelle verbunden ist. Bei der Lichtquelle handelt es sich um eine 50Watt Xenon Bogenlampe, die mit einem optischen Bandpassfilter ausgestattet ist. Lichtquelle, Kamera und Objekttisch wurden mit einem speziellen Tuch abgedunkelt (Abb.4.9), zusätzlich wurde während der Messung die Raumbeleuchtung ausgeschaltet. 


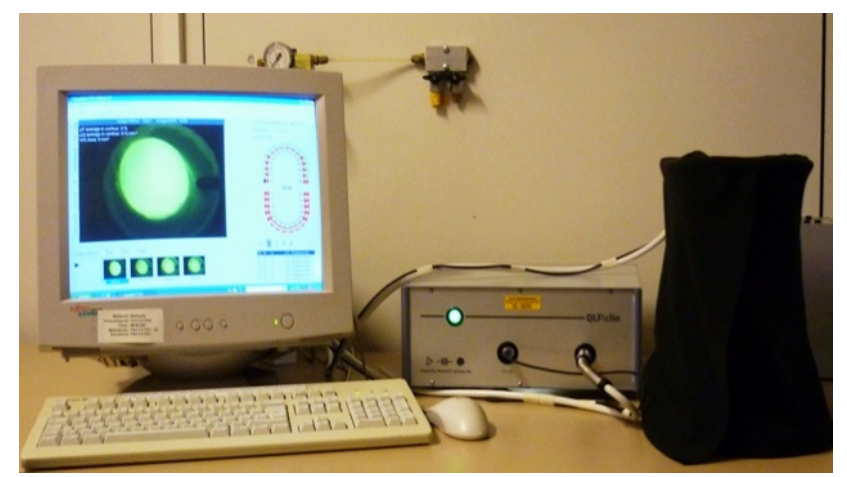

Abb.4.9 QLF- Apparatur mit PC, angeschlossenem QLF- Auslesegerät, Kamera und Lichtquelle (Kamera und Lichtquelle werden von dem Verdunkelungstuch bedeckt)

Die Prüfkörper wurden vor den Messungen 20 Minuten getrocknet. Anschließend erfolgte die Positionierung des jeweiligen Prüfkörpers mittig auf dem Objekttisch, die schwarz markierte Einkerbung des Prüfkörpers wies dabei auf die Drei- Uhr- Position. Der Objekttisch verfügt über vier Freiheitsgrade zur Positionierung der Proben, sodass die Prüfkörper optimal zur Kamera und der Lichtquelle ausgerichtet werden können. Für jeden Prüfkörper wurde ein Einzelbild zur Baseline angefertigt und gespeichert, wobei jedes Bild dem entsprechenden Prüfkörper und dem Messzeitpunkt eindeutig und reproduzierbar zugeordnet wurde. Nach der Messung wurden die Prüfkörper wieder umgehend in $\mathrm{NaCl}$ - Lösung gelagert. Die auf diese Weise erstellten Einzelbilder wurden als Referenzbilder bei den späteren QLFMessungen verwendet.

An den Versuchstagen 7, 14 und 30 wurden erneut Einzelbilder aller Prüfkörper mit der QLF erstellt. Das QLF- System verfügt über eine Auto- Capture- Funktion. Diese Funktion vergleicht während der Prüfkörperpositionierung kontinuierlich das Videobild live mit dem bei der Baseline erstellten Referenzbild. Sobald eine zufriedenstellende Kongruenz zwischen den beiden Bildern erreicht ist, wird automatisch ein Einzelbild angefertigt. Nach der letzten Messung am Versuchstag 30 erfolgte die Analyse mit der QLF. Dazu wurde auf dem Referenzbild ein Analysebereich markiert, der anschließend von der QLF- Software zu allen Messzeitpunkten hinsichtlich der prozentualen Fluoreszenzabnahme und der WS Area ausgewertet wurde. 


\subsubsection{Applikation der Testprodukte}

Am ersten Tag der Versuchsphase erfolgte die Applikation der Testprodukte. Alle Produkte wurden einmalig zu Beginn des ersten Versuchstages appliziert, bevor die Prüfkörper zum ersten Mal in der Remineralisationslösung gelagert wurden. Das nach Herstellerangaben angewendete Applikationsverfahren wird im Folgenden für jedes Testprodukt beschrieben:

\section{Cervitec Plus ${ }^{\circledR}$}

Der Lack wurde nach Trockenlegung der gereinigten Schmelzoberfläche aufgetragen. Dazu wurden zunächst drei Tropfen des Lackes in ein Dappenglas gegeben und eine dünne Lackschicht mit Hilfe einer Mikro- Bürste extra- fine (Kerr GmbH, Rastatt, Deutschland) appliziert. Anschließend wurde der Lack getrocknet und verblasen.

\section{Maximum Cure ${ }^{\circledR}$}

Zunächst wurden die Schmelzoberflächen der Prüfkörper 30 Sekunden lang mit dem 37prozentigen Phosphorsäure- Ätzgel Total Etch (Ivoclar Vivadent AG, Schaan, Lichtenstein) konditioniert und anschließend so lange mit destilliertem Wasser abgespült, bis das Ätzgel vollständig entfernt war. Anschließend wurden die Schmelzoberflächen getrocknet. Die beiden Komponenten des Sealers wurden in einem Dappenglas vermischt und mit Hilfe einer Mikro- Bürste extra- fine (Kerr $\mathrm{GmbH}$, Rastatt, Deutschland) in einer dünnen, gleichmäßigen Schicht auf die geätzten Schmelzoberflächen appliziert.

\section{Pro Sealß}

Auch bei diesem Sealer war eine Konditionierung der Schmelzoberflächen mit dem 37prozentigen Phosphorsäure- Ätzgel Total Etch (Ivoclar Vivadent AG, Schaan, Lichtenstein) erforderlich. Im Anschluss daran wurden die Prüfkörper so lange mit destilliertem Wasser abgespült, bis das Ätzgel vollständig entfernt war. Die Prüfkörper wurden anschließend getrocknet. Zunächst wurden drei Tropfen des Sealers in ein Dappenglas gegeben und danach mit Hilfe einer Mikro- Bürste extra- fine (Kerr GmbH, Rastatt, Deutschland) in einer dünnen, gleichmäßigen Schicht auf die geätzten Schmelzoberflächen appliziert. Abschließend wurde die Sealerschicht für 20 Sekunden mit einer Halogenlampe (Ortholux ${ }^{\mathrm{TM}}$ XT Curing Light, 3M Unitek, Langsberg, Deutschland) lichtgehärtet. 


\subsubsection{Versuchszyklen}

Nach der Produktapplikation begann der Zyklus des ersten Versuchstages.

Pro Gruppe standen täglich jeweils zwei autoklavierte Schraubdeckeldosen zur Verfügung (Abb.4.10); eine befüllt mit 20ml der Remineralisationslösung (künstlicher Speichel), die andere befüllt mit 20ml der Demineralisationslösung (Glucose- Bouillon mit Bakterien, 23 Stunden zuvor angesetzt). Für die Negativkontrolle wurde die entsprechende Schraubdeckeldose anstelle der Demineralisationslösung mit der Glucose- Bouillon (ohne Bakterien) befüllt. Die Bouillon wurde morgens, der künstliche Speichel abends täglich durch frische Lösungen ersetzt, wobei bei jedem Wechsel autoklavierte Schraubdeckeldosen eingesetzt wurden.
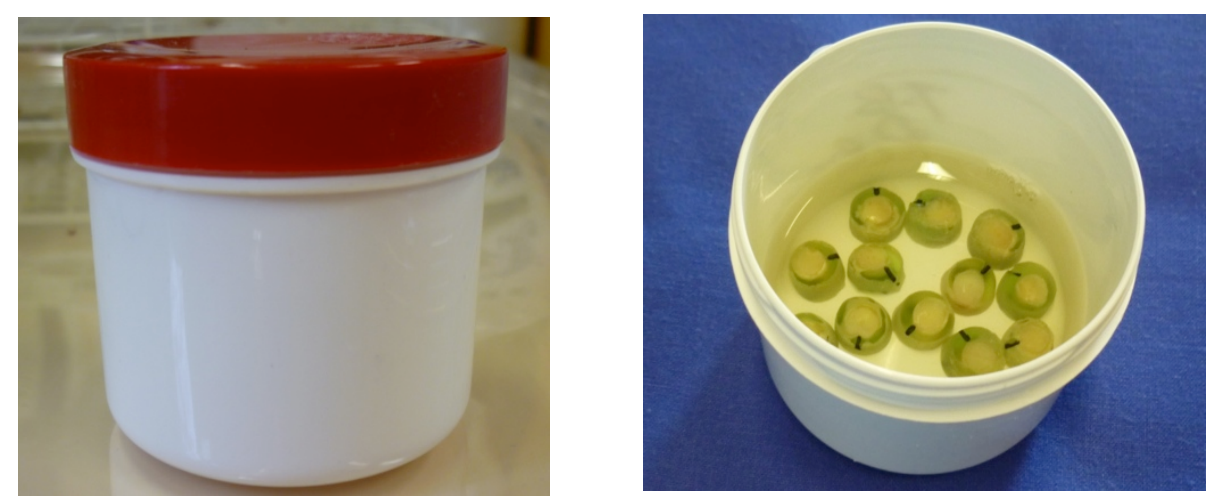

Abb.4.10 Schraubdeckeldose, verschlossen und ohne Deckel mit darin gelagerten Prüfkörpern

Zunächst wurden die Prüfkörper der jeweiligen Gruppen eine Stunde lang in der Remineralisationslösung gelagert. Anschließend wurden die Prüfkörper gruppenweise alternierend für jeweils eine Stunde in der De- und Remineralisationslösung gelagert. Das Umsetzen der Prüfkörper erfolgte mit sterilen Einmalpinzetten (Pflegebedarf24.de - E. Bister \& J. Lutermann GbR, Wermelskirchen, Deutschland). Dieser Zyklus der abwechselnden De- und Remineralisation wurde dreimal täglich wiederholt, sodass die Prüfkörper pro Versuchstag jeweils drei Stunden der Demineralisationslösung ausgesetzt wurden. Während der De- und Remineralisationsphasen wurden die entsprechenden Schraubdeckeldosen mit den Prüfkörpern in einem Gaspacktopf und einem Anaerobierpad in einem auf $36,7^{\circ} \mathrm{C}$ temperierten Brutraum gelagert. Am Ende des Versuchstages wurden alle Prüfkörper mit destilliertem Wasser abgespült und anschließend gruppenweise in neuen Schraubdeckeldosen mit frischem künstlichen Speichel über Nacht gelagert. 
Diese Versuchszyklen wurden an allen 30 Tagen der Versuchsphase wiederholt. Zusätzlich wurden an den Versuchstagen $\mathrm{t} 1=7, \mathrm{t} 2=14$ und $\mathrm{t} 3=30$ nach Durchlaufen der Zyklen erneut Messungen mit der QLF durchgeführt.

\subsection{Statistische Auswertung}

Die statistische Auswertung wurde in Zusammenarbeit mit der Abteilung für medizinische Statistik der Universitätsmedizin Göttingen durchgeführt.

Die erfassten Daten wurden mit Hilfe des Tabellenkalkulationsprogramms Microsoft@ Excel 2003 (Microsoft Corporation, Redmond, USA) aus der QLF- Software exportiert und erfasst. Die statistischen Analysen wurden mit den lizensierten Softwareprogrammen Statistica (Version 9, StatSoft (Europe) GmbH, Hamburg, Deutschland) und SAS (Version 9.2, SAS Institute $\mathrm{GmbH}$, Heidelberg, Deutschland) ausgeführt. Der Effekt des Testproduktes bzw. der Zeit (bzw. deren Wechselwirkung) auf den Fluoreszenzverlust und die WS Area wurden mittels nichtparametrischer 2- faktorieller Varianzanalyse untersucht. Ließ sich ein globaler signifikanter Effekt ermitteln, wurden weitere Paarvergleiche mittels Wilcoxon- Mann- WhitneyTest (WMW- Test) durchgeführt. Für alle globalen Tests wurde als Signifikanzniveau $a=5 \%$ und für die Paarvergleiche das Bonferroni- korrigierte Niveau von a durch Testanzahl festgelegt. Des Weiteren wurde eine Korrelationsberechnung der betrachteten Parameter prozentuale Fluoreszenzabnahme und WS Area durchgeführt (Bestimmung des Korrelationskoeffizienten nach Pearson). 


\section{Ergebnisse}

Die Ergebnisse der QLF- Messungen werden im Folgenden beschrieben und grafisch mittels Box- and-Whiskers Plots dargestellt. Die obere und untere Begrenzungslinie der Box wird durch das obere und untere Quartil der Datenwerte festgelegt. Der Interquartilsabstand (IQR) beschreibt den Wertebereich, in dem die mittleren fünfzig Prozent der Daten liegen. Dieser wird durch die Ausdehnung der Box ausgedrückt. An das obere und untere Ende der Box schließen sich die Whiskers an, wobei die Enden jeweils den höchsten beziehungsweise niedrigsten Wert innerhalb des 1,5- Fachen Interquartilsabstandes darstellen. Der Median wird durch eine Markierung in der Box ( $\square$ ) ausgedrückt. Werte, die um mehr als das 1,5Fache des Interquartilsabstandes außerhalb liegen, werden als Ausreißer ( $\circ$ ) bezeichnet, und Werte, die um mehr als das 3- Fache des Interquartilsabstandes außerhalb liegen, werden als Extreme (*) bezeichnet. In den nachfolgenden Abbildungen sind auf der Ordinate die prozentuale Fluoreszenzabnahme in \%, respektive die WS Area in $\mathrm{mm}^{2}$ und auf der Abszisse die Gruppen aufgetragen.

Die mit dem QLF untersuchten Parameter prozentuale Fluoreszenzabnahme $(\Delta \mathrm{F})$ und WS Area werden nachfolgend zunächst separat beschrieben. Für jede Produktgruppe wird die Entwicklung im zeitlichen Verlauf betrachtet, um festzustellen, ob und in welchem Maß es zu Fluoreszenzabnahmen beziehungsweise Zunahmen der WS Area innerhalb der Gruppe kam. Anschließend erfolgen ein Vergleich der Produktgruppen zu den Kontrollgruppen und Vergleiche zwischen den Produktgruppen untereinander, um zu ermitteln, inwieweit die Produkte einen signifikanten Einfluss auf die Fluoreszenzabnahme und WS Area nahmen und ob signifikante Unterschiede zwischen den Testprodukten feststellbar waren. Abschließend wird geprüft, inwiefern die untersuchten Parameter prozentuale Fluoreszenzabnahme und WS Area miteinander korreliert waren.

\subsection{Prozentuale Fluoreszenzabnahme $(\Delta \mathrm{F})$}

Die Ergebnisse der globalen Tests zeigten, dass signifikante Veränderungen der prozentualen Fluoreszenz bei den Versuchsgruppen zu verzeichnen waren $(p<0.001)$. Es wurde weiterhin ersichtlich, dass es ebenfalls im zeitlichen Verlauf zu einer signifikanten Fluoreszenzabnahme kam $(p<0.001)$. Zudem lag eine signifikante Wechselwirkung zwischen den Gruppen und der Zeit vor $(\mathrm{p}<0.001)$. 


\subsubsection{Innerhalb der Gruppen}

Mit Ausnahme der Negativkontrollgruppe konnten für jede Gruppe signifikante Fluoreszenzabnahmen im Verlauf der Studie festgestellt werden $(p<0.05)$. In Tabelle 5.1 sind die ermittelten Daten der statistischen Auswertung zu den verschiedenen Messzeitpunkten sowie die adjustierten $\mathrm{p}$ - Werte für den Vergleich zwischen Baseline und dem Messzeitpunkt nach 30 Tagen für jede Gruppe aufgelistet.

Tab.5.1 Prozentuale Fluoreszenzabnahme der einzelnen Gruppen zu den verschiedenen Messzeitpunkten. Alle Werte stellen die Differenz zur Baseline dar. ( ${ }^{*}$ signifikante $p$-Werte, $p$ nach Bonferroni adjustiert)

\begin{tabular}{|c|c|c|c|c|c|}
\hline $\begin{array}{l}\text { Produkt- } \\
\text { gruppe }\end{array}$ & $\begin{array}{l}\text { Messzeit- } \\
\text { punkt }\end{array}$ & Median & $\begin{array}{l}\text { Interquartils- } \\
\text { abstand (IQR) }\end{array}$ & Range & $\begin{array}{l}\text { Adj. p-Wert } \\
\text { Vergleich: } \\
\text { Baseline-30d }\end{array}$ \\
\hline $\begin{array}{l}\text { Cervitec } \\
\text { Plus }{ }^{\circledR}\end{array}$ & $\begin{array}{l}\text { Baseline } \\
7 d \\
14 d \\
30 d\end{array}$ & $\begin{array}{r}0.00 \\
0.00 \\
-5.94 \\
-8.91 \\
\end{array}$ & $\begin{array}{r}0.00-0.00 \\
-5.69-0.00 \\
-6.49-0.00 \\
-12.60-(-8.04) \\
\end{array}$ & $\begin{array}{r}0.00-0.00 \\
-9.49-0.00 \\
-7.97-0.00 \\
-15.60-(-5.96) \\
\end{array}$ & $0.014^{*}$ \\
\hline $\begin{array}{l}\text { Maximum } \\
\text { Cure } \AA\end{array}$ & $\begin{array}{l}\text { Baseline } \\
7 d \\
14 d \\
30 d\end{array}$ & $\begin{array}{l}0.00 \\
0.00 \\
0.00 \\
0.00\end{array}$ & $\begin{array}{r}0.00-0.00 \\
-7.10-0.00 \\
-7.79-0.00 \\
-11.00-0.00\end{array}$ & $\begin{array}{r}0.00-0.00 \\
-9.49-0.00 \\
-9.20-0.00 \\
-19.70-0.00\end{array}$ & 0.252 \\
\hline Pro Seal® & $\begin{array}{l}\text { Baseline } \\
7 d \\
14 d \\
30 d\end{array}$ & $\begin{array}{r}0.00 \\
0.00 \\
-5.93 \\
0.00 \\
\end{array}$ & $\begin{array}{r}0.00-0.00 \\
-6.91-0.00 \\
-7.65-0.00 \\
-8.53-0.00 \\
\end{array}$ & $\begin{array}{r}0.00-0.00 \\
-9.90-0.00 \\
-13.50-0.00 \\
-21.90-0.00 \\
\end{array}$ & 0.392 \\
\hline $\begin{array}{l}\text { Positiv- } \\
\text { kontrolle }\end{array}$ & $\begin{array}{l}\text { Baseline } \\
7 d \\
14 d \\
30 d\end{array}$ & $\begin{array}{r}0.00 \\
-5.84 \\
-7.51 \\
-11.80 \\
\end{array}$ & $\begin{array}{r}0.00-0.00 \\
-6.45-0.00 \\
-9.08-(-6.80) \\
-17.00-(-9.83) \\
\end{array}$ & $\begin{array}{r}0.00-0.00 \\
-9.70-0.00 \\
-9.79-(-5.90) \\
-20.50-(-7.06) \\
\end{array}$ & $0.014^{*}$ \\
\hline $\begin{array}{l}\text { Negativ- } \\
\text { kontrolle }\end{array}$ & $\begin{array}{l}\text { Baseline } \\
7 d \\
14 d \\
30 d\end{array}$ & $\begin{array}{l}0.00 \\
0.00 \\
0.00 \\
0.00 \\
\end{array}$ & $\begin{array}{c}0.00-0.00 \\
0.00-0.00 \\
0.00-0.00 \\
0.00-0.00 \\
\end{array}$ & $\begin{array}{c}0.00-0.00 \\
0.00-0.00 \\
0.00-0.00 \\
0.00-0.00 \\
\end{array}$ & - \\
\hline
\end{tabular}

Im Folgenden wird die Entwicklung der prozentualen Fluoreszenzabnahme für jede Gruppe kurz beschrieben.

Cervitec Plus ${ }^{\circledR}$ wies nach 14 Tagen erstmals eine deutliche Abnahme der prozentualen Fluoreszenz auf (Median -5.95, IQR -6.49-0.00). Nach 30 Tagen wurde die deutlichste Fluoreszenzabnahme mit einem Median von -8.91 (IQR -12.60-(-)8.04) ermittelt, und es lag ein signifikanter Unterschied zur Baseline vor $(p=0.014)$. Maximum Cure ${ }^{\circledR}$ wies zu allen Messzeitpunkten stets einen Median von 0.00 (IQR nach 30 Tagen -11.00-0.00) auf. Auch nach 30 
Tagen konnte bei dieser Produktgruppe keine signifikante Zunahme der prozentualen Fluoreszenzabnahme im Vergleich zur Baseline festgestellt werden $(p=0.252)$. Bei den Ergebnissen von Pro Sealß war ungewöhnlich, dass der Median nach 14 Tagen bei -5.93 (IQR -7.650.00) lag, während nach 30 Tagen ein Median von 0.00 (IQR -8.53-0.00) ermittelt wurde. Bei dem Vergleich des Messzeitpunktes nach 30 Tagen zur Baseline konnte bei Pro Seal® keine signifikante Abnahme der prozentualen Fluoreszenz festgestellt werden $(p=0.392)$. Bei der Positivgruppe wurde die deutlichste Fluoreszenzabnahme festgestellt. Bereits nach 7 Tagen lag ein Median von -5.84 (IQR -6.45-0.00) vor, und im weiteren Verlauf kam es zwischen den Messzeitpunkten zu einer kontinuierlichen Abnahme der prozentualen Fluoreszenz. Nach 30 Tagen wurde ein Median von -11.80 (IQR -17.00-(-)9.83) ermittelt. Beim Vergleich des Messzeitpunktes nach 30 Tagen mit der Baseline konnte eine signifikante Abnahme der prozentualen Fluoreszenz bei der Positivkontrolle festgestellt werden $(p=0.014)$. Abbildung 5.1 gibt einen Überblick über die erstellten Vergleiche der einzelnen Gruppen zwischen Baseline und der Messung nach 30 Tagen.

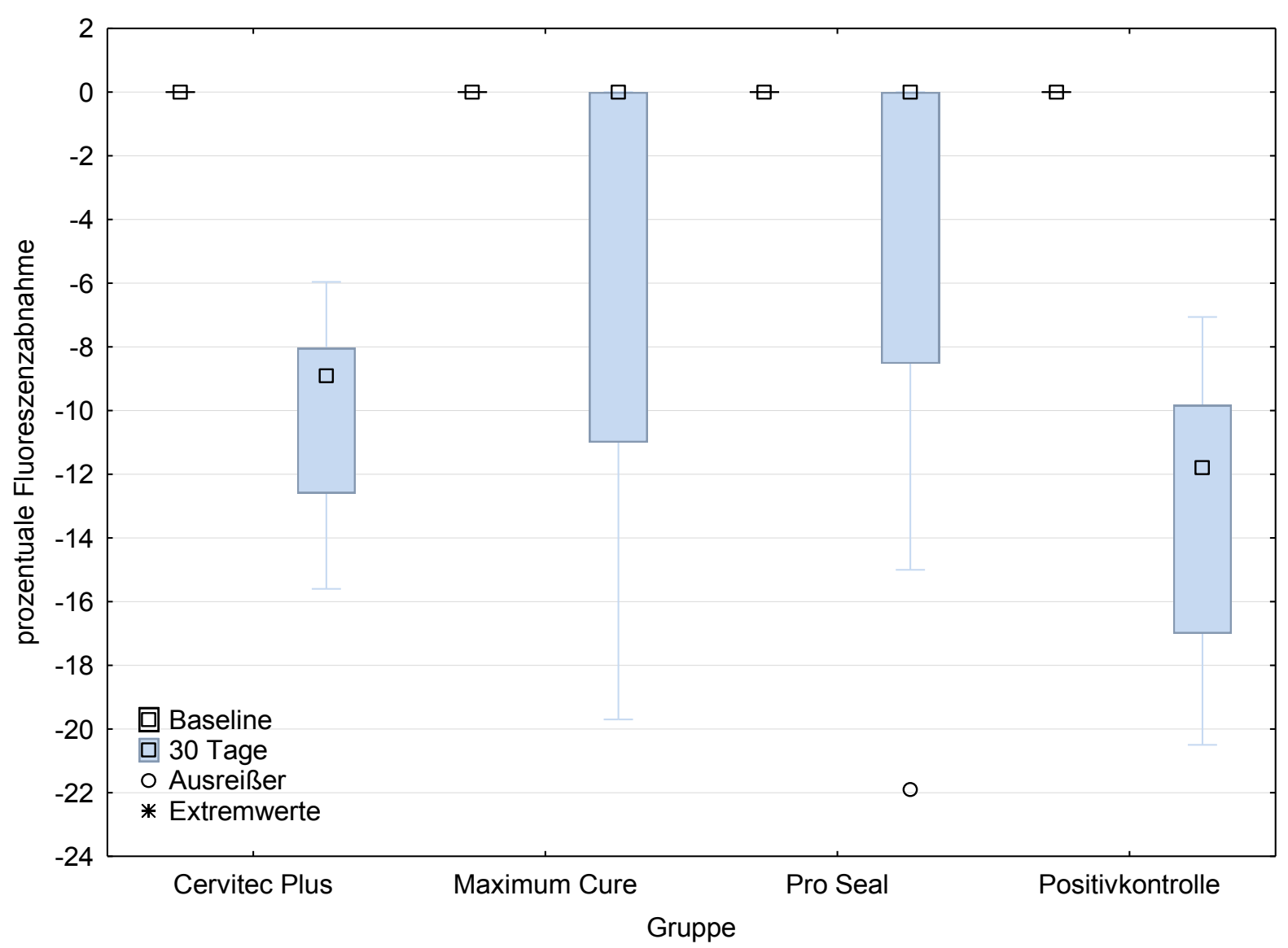

Abb.5.1 Box- Plot der prozentualen Fluoreszenzabnahme im Vergleich zwischen Baseline und dem Messzeitpunkt nach 30 Tagen der einzelnen Gruppen 


\subsubsection{Im Vergleich zu den Kontrollgruppen}

Bei der Negativkontrolle, bei der weder Produkte appliziert noch eine Demineralisationslösung mit Streptokokken eingesetzt wurden, konnte während der gesamten Versuchsphase keine Abnahme der prozentualen Fluoreszenz festgestellt werden. Mit Ausnahme von Cervitec Plus ${ }^{\circledR}$ lagen bei allen Vergleichen der Negativkontrolle mit den anderen Produkten nach 30 Tagen keine signifikanten Unterschiede vor $(p=1.000)$. Cervitec Plus ${ }^{\circledR}$ wies nach 30 Tagen eine signifikant deutlichere Abnahme der prozentualen Fluoreszenz auf $(p=0.014)$.

Bei der Positivkontrolle, bei der zwar die Demineralisationslösung mit Streptokokken, nicht aber ein Produkt appliziert wurde, konnte die deutlichste Fluoreszenzabnahme festgestellt werden. Auch diese Kontrollgruppe wurde nach 30 Tagen mit den Produktgruppen verglichen.

Die statistischen Vergleiche zeigten, dass es bei allen Gruppen mit Ausnahme von Cervitec Plus ${ }^{\circledR}$ zu einer signifikant geringeren Zunahme der prozentualen Fluoreszenz kam als bei der Positivkontrolle ( $p<0.05)$. Cervitec Plus ${ }^{\circledR}$ unterschied sich nach 30 Tagen nicht signifikant von der Positivkontrolle ( $p=0.644)$. Die Negativ- und Positivkontrolle unterschieden sich nach 30 Tagen signifikant voneinander $(p=0.014)$. Der Tabelle 5.2 sind die ermittelten $p$ - Werte für jeden Vergleich zu entnehmen, die Abbildung 5.2 veranschaulicht die erstellten Vergleiche grafisch.

Tab.5.2 p- Werte der statistischen Vergleiche der prozentualen Fluoreszenzabnahme zwischen den Kontrollgruppen und den Produktgruppen nach 30 Tagen ( ${ }^{*}$ signifikante $\mathrm{p}$ - Werte, p nach Bonferroni adjustiert)

\begin{tabular}{|c|c|c|}
\hline Kontrollgruppe & Vergleich mit & adj. p-Wert \\
\hline \multirow[t]{3}{*}{ Negativkontrolle } & Cervitec Plus ${ }^{\circledR}$ & $0.014^{\star}$ \\
\hline & Maximum Cure $\AA$ & 1.000 \\
\hline & Pro Seal ${ }^{\circledR}$ & 1.000 \\
\hline \multirow[t]{4}{*}{ Positivkontrolle } & Cervitec Plus ${ }^{\circledR}$ & 0.644 \\
\hline & Maximum Cure ${ }^{\circledR}$ & $0.014^{*}$ \\
\hline & Pro Seal® & $0.028^{*}$ \\
\hline & Negativkontrolle & $0.014^{*}$ \\
\hline
\end{tabular}




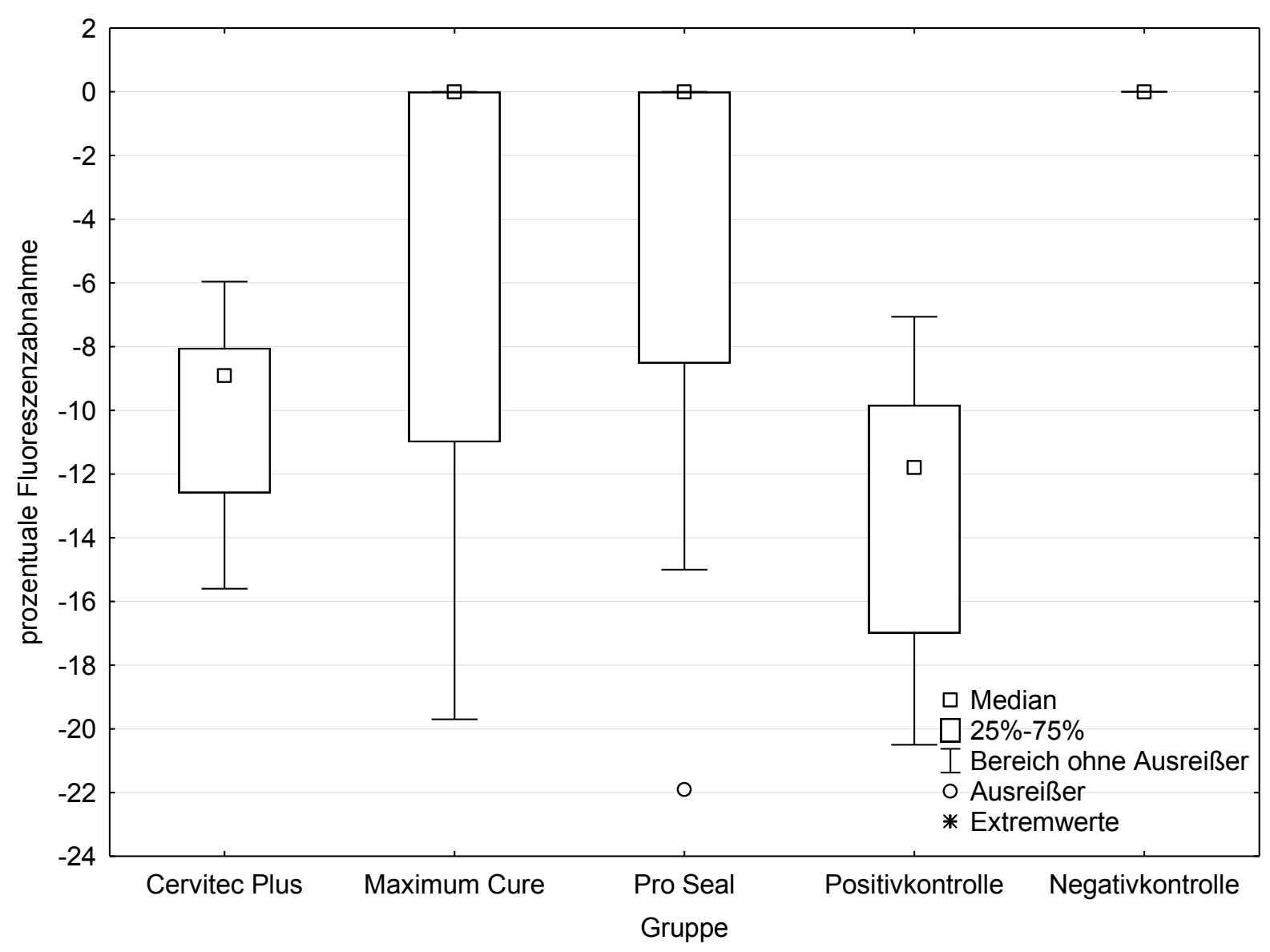

Abb.5.2 Box- Plot der prozentualen Fluoreszenzabnahme der verschiedenen Gruppen nach 30 Tagen

\subsubsection{Vergleich der Produktgruppen}

Die Ergebnisse der QLF- Messungen zeigten, dass nach 30 Tagen keine signifikanten Unterschiede hinsichtlich der prozentualen Fluoreszenzabnahme zwischen den getesteten Produkten vorlagen $(p>0.05)$. Während bei den Produkten Maximum Cure ${ }^{\circledR}$ und Pro Seal® jedoch nach 30 Tagen ein Median von 0.00 (IQR -11.00-0.00 bzw. -8.53-0.00) vorlag, wies Cervitec Plus ${ }^{\circledR}$ tendenziell schlechtere Werte auf (Median nach 30 Tagen -8.91, IQR -12.60(-)8.04). Die Tabelle 5.3 zeigt die p-Werte der Produktvergleiche, Abbildung 5.2 veranschaulicht die erstellten Vergleiche grafisch.

Tab.5.3 p- Werte der statistischen Vergleiche der prozentualen Fluoreszenzabnahme zwischen den Produktgruppen nach 30 Tagen ( ${ }^{*}$ signifikante $p$ - Werte, $p$ nach Bonferroni adjustiert)

\begin{tabular}{|c|c|}
\hline Produktvergleich & adj. p-Wert \\
\hline Cervitec Plus ${ }^{\circledR}$ vs. Maximum Cure ${ }^{\circledR}$ & 0.266 \\
\hline Cervitec Plus ${ }^{\circledR}$ vs. Pro Seal ${ }^{\circledR}$ & 0.098 \\
\hline Maximum Cure ${ }^{\circledR}$ vs. Pro Seal ${ }^{\circledR}$ & 1.000 \\
\hline
\end{tabular}




\subsection{Entwicklung der WS Area}

Auch bei den Ergebnissen der globalen Tests für die WS Area zeigte sich, dass signifikante Veränderungen bei den Versuchsgruppen zu verzeichnen waren $(p<0.001)$. Es wurde weiterhin ersichtlich, dass es ebenfalls im zeitlichen Verlauf zu einer signifikanten Zunahme der WS Area kam $(p<0.001)$ und eine signifikante Wechselwirkung zwischen den Gruppen und der Zeit vorlag $(p<0.001)$.

\subsubsection{Innerhalb der Gruppen}

Mit Ausnahme der Negativkontrollgruppe konnte für jede Gruppe eine signifikante Zunahme der WS Area im Verlauf der Studie festgestellt werden $(p<0.05)$. Die Tabelle 5.4 gibt einen Überblick über die Ergebnisse zu den verschiedenen Messzeitpunkten sowie über die adjustierten $p$ - Werte der Vergleiche zwischen Baseline und dem Messzeitpunkt nach 30 Tagen.

Tab.5.4 Vergleich der WS Area zwischen den 6 Versuchsgruppen und den 4 Zeitpunkten. Alle Werte stellen die Differenz zur Baseline dar. ( ${ }^{*}$ signifikante $p$ - Werte, $p$ nach Bonferroni adjustiert)

\begin{tabular}{|l|l|r|r|r|r|}
\hline $\begin{array}{l}\text { Produkt- } \\
\text { gruppe }\end{array}$ & $\begin{array}{l}\text { Messzeit- } \\
\text { punkt }\end{array}$ & Median & $\begin{array}{l}\text { Interquartils- } \\
\text { abstand (IQR) }\end{array}$ & $\begin{array}{c}\text { Range } \\
\text { Adj. p-Wert } \\
\text { Vergleich: } \\
\text { Baseline-30d }\end{array}$ \\
\hline Cervitec & Baseline & 0.00 & $0.00-0.00$ & $0.00-0.00$ & $0.014^{\star}$ \\
Plus ${ }^{\circledR}$ & 7d & 0.00 & $0.00-0.00$ & $0.00-0.01$ & \\
& 14d & 0.00 & $0.00-0.03$ & $0.00-3.48$ & \\
\hline Maximum & 30d & 3.49 & $0.10-7.95$ & $0.03-13.10$ & 0.252 \\
Cure ${ }^{\circledR}$ & Baseline & 0.00 & $0.00-0.00$ & $0.00-0.00$ & \\
& 7d & 0.00 & $0.00-0.02$ & $0.00-0.11$ & \\
& 14d & 0.00 & $0.00-0.01$ & $0.00-0.14$ & \\
\hline Pro Seal ${ }^{\circledR}$ & Baseline & 0.00 & $0.00-0.02$ & $0.00-0.28$ & \\
& 7d & 0.00 & $0.00-0.00$ & $0.00-0.00$ & \\
& 14d & 0.00 & $0.00-0.00$ & $0.00-0.55$ & \\
& 30d & 0.00 & $0.00-0.10$ & $0.00-0.34$ & \\
\hline Positiv- & Baseline & 0.00 & $0.00-0.05$ & $0.00-0.56$ & \\
kontrolle & 7d & 0.00 & $0.00-0.00$ & $0.00-0.00$ & \\
& 14d & 0.00 & $0.00-0.21$ & $0.00-0.62$ & \\
& 30d & 1.63 & $0.02-3.81$ & $0.00-8.77$ & \\
\hline Negativ- & Baseline & 7.67 & $3.16-10.60$ & $0.65-15.90$ & \\
kontrolle & 7d & 0.00 & $0.00-0.00$ & $0.00-0.00$ & \\
& 14d & 0.00 & $0.00-0.00$ & $0.00-0.00$ & \\
& 30d & 0.00 & $0.00-0.00$ & $0.00-0.00$ & \\
\hline
\end{tabular}


Nach 30 Tagen wies Cervitec Plus ${ }^{\circledR}$ eine deutliche Zunahme der WS Area mit einem Median von 3.49 (IQR 0.10-7.95) auf und es lag ein signifikanter Unterschied zur Baseline vor $(p=0.014)$. Der Median lag zu allen vorherigen Messzeitpunkten bei 0.00. Bei Maximum Cure ${ }^{\circledR}$ und Pro Seal ${ }^{\circledR}$ wurden nach 30 Tagen keine signifikanten Zunahmen der WS Area im Vergleich zur Baseline festgestellt $(p=0.252$ bzw. $p=0.952)$, und der Median lag zu allen Messzeitpunkten stets bei 0.00 (IQR nach 30 Tagen 0.00-0.02 bzw. 0.00-0.05). Die Positivgruppe wies die größte Zunahme der WS Area auf (Median nach 30 Tagen 7.67 (IQR 3.1610.60)), und bei der Messung nach 30 Tagen wurde eine signifikante Zunahme der WS Area im Vergleich zu der Baseline ermittelt $(p=0.014)$. In der Abbildung 5.3 sind die Messzeitpunktvergleiche grafisch dargestellt.

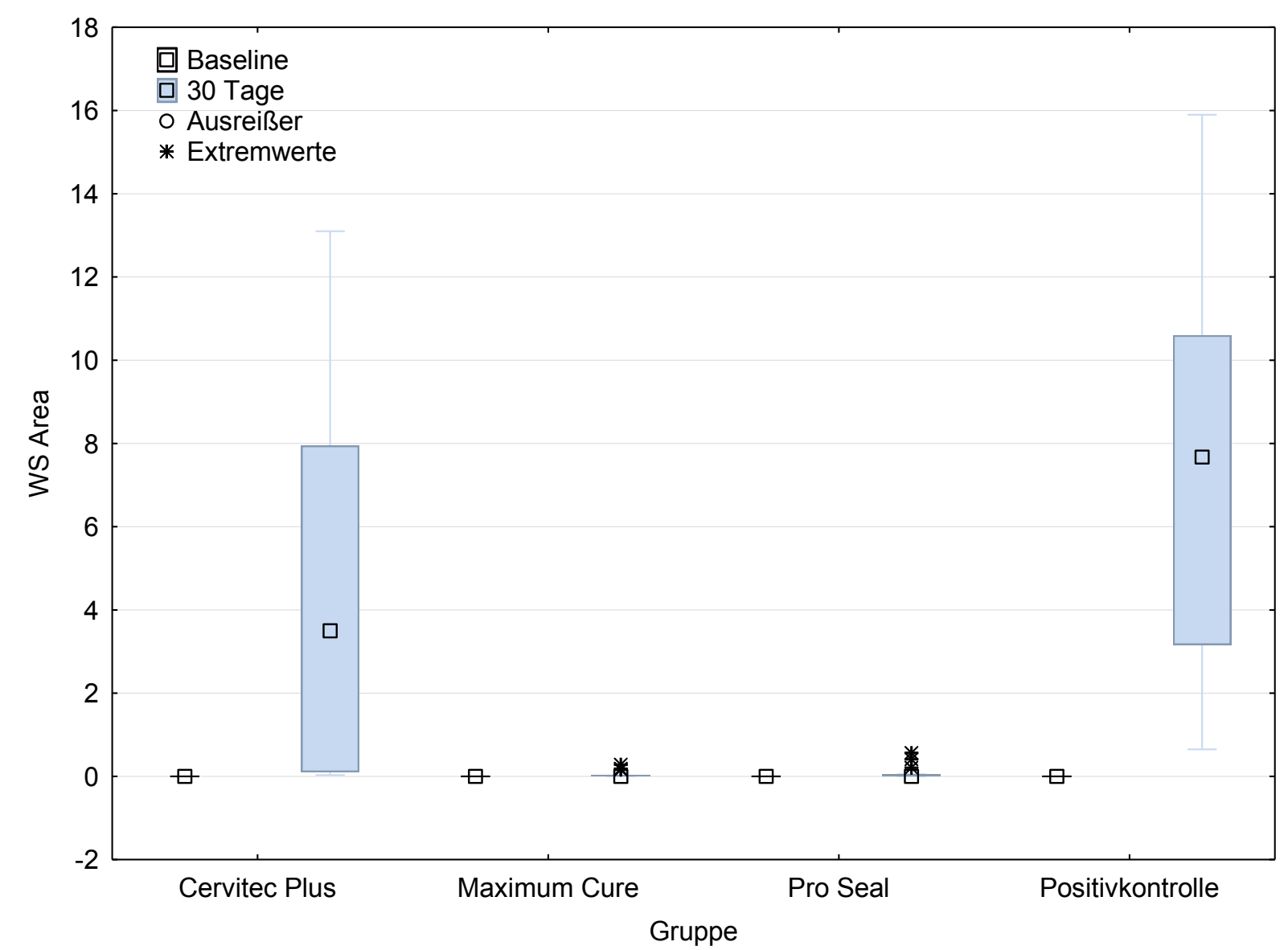

Abb.5.3 Box- Plot der Vergleiche zwischen Baseline und dem Messzeitpunkt nach 30 Tagen der einzelnen Gruppen

\subsubsection{Im Vergleich zu den Kontrollgruppen}

Die beiden Kontrollgruppen unterschieden sich nach 30 Tagen signifikant voneinander $(p=0.014)$. Cervitec Plus ${ }^{\circledR}$ wies im Vergleich zu der Negativkontrolle nach 30 Tagen eine signifikant größere Zunahme der WS Area auf. Demgegenüber unterschieden sich Maximum 
Cure ${ }^{\circledR}$ und Pro Seal ${ }^{\circledR}$ nach 30 Tagen nicht signifikant von der Negativkontrolle. Bei der Positivgruppe wurde im Vergleich zu den anderen Produktgruppen die deutlichste Zunahme der WS Area festgestellt. Cervitec Plus ${ }^{\circledR}$ zeigte nach 30 Tagen keinen signifikanten Unterschied zur Positivkontrolle $(p=1.000)$, während die anderen Testprodukte eine signifikant geringere Zunahme der WS Area im Vergleich zur Positivkontrolle aufwiesen $(p<0.05)$. Der Tabelle 5.5 sind die ermittelten $\mathrm{p}$ - Werte für die Vergleiche mit den Kontrollgruppen zu entnehmen, die Abbildung 5.4 gibt einen grafischen Überblick.

Tab.5.5 p- Werte der statistischen Vergleiche der WS Area zwischen den Kontrollgruppen und den Produktgruppen nach 30 Tagen ( ${ }^{*}$ signifikante $p$ - Werte, $p$ nach Bonferroni adjustiert)

\begin{tabular}{|c|c|c|}
\hline Kontrollgruppe & Vergleich mit & adj. p-Wert \\
\hline \multirow[t]{3}{*}{ Negativkontrolle } & Cervitec Plus $\AA$ & $0.014^{*}$ \\
\hline & Maximum Cure ${ }^{\circledR}$ & 1.000 \\
\hline & Pro Seal ${ }^{\circledR}$ & 1.000 \\
\hline \multirow[t]{4}{*}{ Positivkontrolle } & Cervitec Plus ${ }^{\circledR}$ & 1.000 \\
\hline & Maximum Cure $\AA$ & $<0.001^{*}$ \\
\hline & Pro Seal® & $<0.001^{*}$ \\
\hline & Negativkontrolle & $0.014^{*}$ \\
\hline
\end{tabular}

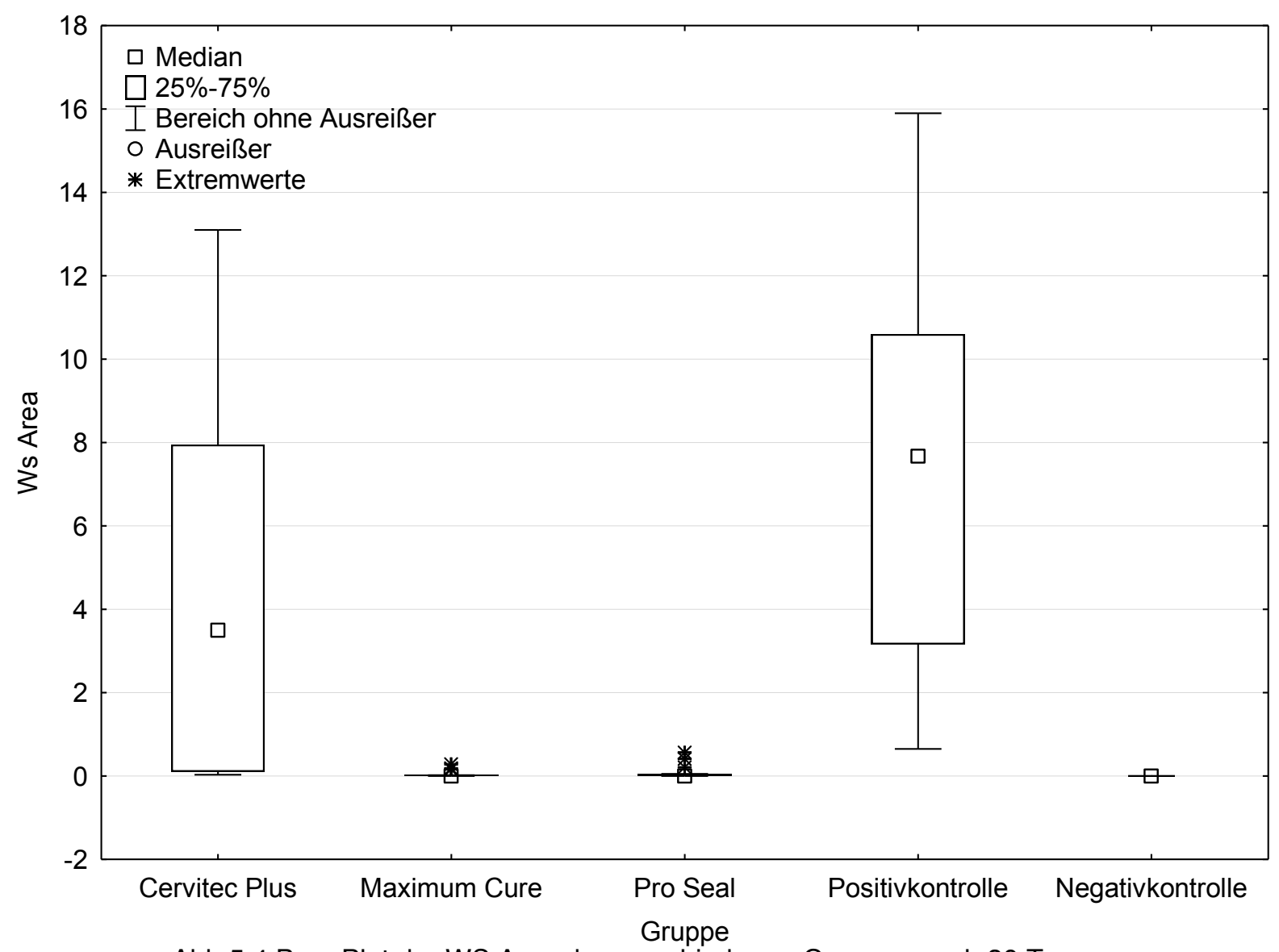

Abb.5.4 Box- Plot der WS Area der verschiedenen Gruppen nach 30 Tagen 


\subsubsection{Vergleich der Produktgruppen}

Cervitec Plus ${ }^{\circledR}$ wies nach 30 Tagen im Vergleich zu den anderen Testprodukten eine signifikant deutlichere Zunahme der WS Area $(p<0.001)$ auf. Pro Seal ${ }^{\circledR}$ und Maximum Cure ${ }^{\circledR}$ unterschieden sich nicht signifikant voneinander $(p=1.000)$. Die Tabelle 5.6 zeigt die ermittelten p- Werte der Produktvergleiche und die Abbildung 5.4 einen grafischen Überblick über die erstellten Gruppenvergleiche.

Tab.5.6 p- Werte der statistischen Vergleiche der WS Area zwischen den Produktgruppen nach 30 Tagen ( ${ }^{*}$ signifikante $p$ - Werte, $p$ nach Bonferroni adjustiert)

\begin{tabular}{|c|c|}
\hline Produktvergleich & adj. p-Wert \\
\hline Cervitec Plus ${ }^{\circledR}$ vs. Maximum Cure ${ }^{\circledR}$ & $<0.001^{*}$ \\
\hline Cervitec Plus ${ }^{\circledR}$ vs. Pro Seal ${ }^{\circledR}$ & $<0.001^{*}$ \\
\hline Maximum Cure ${ }^{\circledR}$ vs. Pro Seal ${ }^{\circledR}$ & 1.000 \\
\hline
\end{tabular}

\subsection{Korrelation von prozentualer Fluoreszenzabnahme $(\Delta F)$ und WS Area}

Im Rahmen der statistischen Auswertung wurde geprüft, ob eine Korrelation der beiden untersuchten Parameter prozentuale Fluoreszenzabnahme und WS Area vorlag.

Der Korrelationskoeffizient $r$ nach Pearson beschreibt die Stärke der linearen Komponente des Zusammenhangs zwischen zwei Parametern und kann Werte zwischen -1 und 1 annehmen. Liegt ein Wert von 1 (bzw. -1) vor, besteht ein vollständig positiver (bzw. negativer) linearer Zusammenhang. Bei einem Wert von 0 besteht dagegen überhaupt kein linearer Zusammenhang zwischen den beiden Parametern. Wenn $r$ - Werte $\geq 0.5$ vorliegen, wird von einem mittleren linearen Zusammenhang, bei $r$ - Werten $\geq 0.8$ von einem stark linearen Zusammenhang ausgegangen. Das Bestimmtheitsmaß $r^{2}$ beschreibt, welcher Anteil der Gesamtvarianz durch die Regressionsgerade erklärt wird, und drückt damit aus, in welchem Umfang die Streuung durch die Regressionsgerade beschrieben wird. Wenn alle Punkte auf der Geraden liegen, besitzt das Bestimmtheitsmaß einen Wert von 1, und es besteht ein vollständiger linearer Zusammenhang zwischen den beiden Parametern (Apostolopoulos et al. 2002).

Bei globaler Betrachtung der Gruppen konnte eine negative signifikante Korrelation zwischen der Zunahme der WS Area und der prozentualen Fluoreszenzabnahme ermittelt werden 
(Abb.5.5), das heißt, große WS Area- Werte gehen mit kleinen $\Delta \mathrm{F}$ - Werten einher und umgekehrt $(r=-0.6115, p=0.000)$. Innerhalb der einzelnen Gruppen wurde eine unterschiedlich starke, jedoch stets signifikante negative Korrelation beider Parameter festgestellt (Tab.5.7).

Tab.5.7 Pearson- Korrelation von $\Delta \mathrm{F}$ und WS Area (* signifikante $\mathrm{p}$ - Werte, $\mathrm{p}$ nach Bonferroni adjustiert)

\begin{tabular}{|l|r|r|r|}
\hline & Korrelationskoeffizient $\mathbf{r}$ & Bestimmtheitsmaß $\mathbf{r}^{\wedge} \mathbf{2}$ & adj. p-Wert \\
\hline Alle Gruppen & -0.6115 & 0,3739 & $0,000^{*}$ \\
\hline Cervitec Plus ${ }^{\circledR}$ & $-0,7293$ & 0.5319 & $0,000^{*}$ \\
\hline Maximum Cure ${ }^{\circledR}$ & $-0,7148$ & 0,5110 & $0,000^{*}$ \\
\hline Pro Seal® & $-0,7782$ & 0,6056 & $0,000^{*}$ \\
\hline Positivkontrolle & $-0,7882$ & 0,6212 & $0,000^{*}$ \\
\hline
\end{tabular}

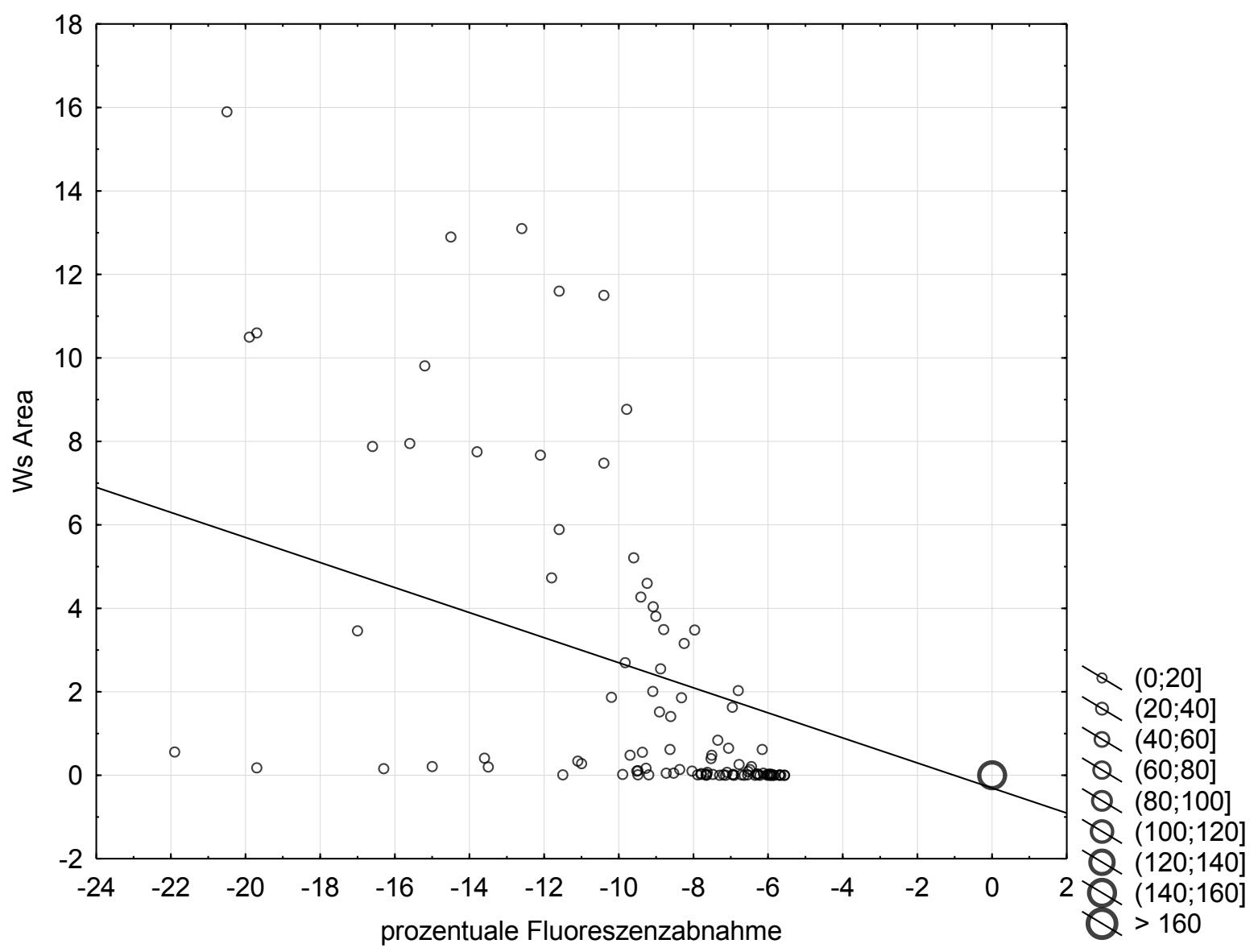

Abb.5.5 Grafische Darstellung der Korrelation von prozentualer Fluoreszenzabnahme und WS Area aller Gruppen unabhängig von der Zeit 


\subsection{Zusammenfassung der wichtigsten Ergebnisse}

\subsubsection{Prozentuale Fluoreszenzabnahme $(\Delta \mathrm{F})$}

- Mit Ausnahme der Negativkontrolle wurden bei allen Gruppen signifikante Abnahmen der prozentualen Fluoreszenz festgestellt.

- Die Positivkontrolle wies nach 30 Tagen die deutlichste prozentuale Fluoreszenzabnahme auf.

- Unter den Testprodukten lag bei Cervitec Plus ${ }^{\circledR}$ die deutlichste Abnahme der prozentualen Fluoreszenz vor. Im Gegensatz zu Maximum Cure ${ }^{\circledR}$ und Pro Seal ${ }^{\circledR}$ wies es nach 30 Tagen eine signifikante Abnahme der prozentualen Fluoreszenz im Vergleich zur Baseline auf.

- Cervitec Plus ${ }^{\circledR}$ zeigte nach 30 Tagen eine signifikant deutlichere Abnahme der prozentualen Fluoreszenz als die Negativkontrolle, im Vergleich zu der Positivkontrolle wurde nach 30 Tagen kein signifikanter Unterschied festgestellt.

- Maximum Cure ${ }^{\circledR}$ und Pro Seal ${ }^{\circledR}$ zeigten nach 30 Tagen eine signifikant geringere Abnahme der prozentualen Fluoreszenz im Vergleich zu der Positivkontrolle und unterschieden sich nicht signifikant von der Negativkontrolle.

- Nach 30 Tagen lagen keine signifikanten Unterschiede zwischen den Testprodukten vor. Cervitec Plus ${ }^{\circledR}$ wies jedoch tendenziell schlechtere Werte auf als Maximum Cure ${ }^{\circledR}$ und Pro Seal ${ }^{\circledR}$

\subsubsection{WS Area}

- Mit Ausnahme der Negativkontrolle wurden bei allen Versuchsgruppen signifikante Zunahmen der WS Area festgestellt.

- Nach 30 Tagen wies die Positivkontrolle die größte Zunahme der WS Area auf.

- Unter den Produktgruppen war bei Cervitec Plus ${ }^{\circledR}$ die größte Zunahme der WS Area zu verzeichnen. Im Gegensatz zu Maximum Cure® und Pro Seal ${ }^{\circledR}$ wies es nach 30 Tagen eine signifikante Zunahme der WS Area im Vergleich zur Baseline auf.

- Nach 30 Tagen wies Cervitec Plus ${ }^{\circledR}$ eine signifikant deutlichere Zunahme der WS Area auf als die Negativkontrolle, während es sich nicht signifikant von der Positivkontrolle unterschied.

- Maximum Cure ${ }^{\circledR}$ und Pro Seal ${ }^{\circledR}$ zeigten nach 30 Tagen eine signifikant geringere Zunahme der WS Area im Vergleich zu der Positivkontrolle und unterschieden sich nicht signifikant von der Negativkontrolle. 
- Bei den Produktgruppenvergleichen lag bei Cervitec Plus ${ }^{\circledR}$ im Vergleich zu Maximum Cure ${ }^{\circledR}$ und Pro Seal ${ }^{\circledR}$ nach 30 Tagen eine signifikant größere Zunahme der WS Area vor.

\subsubsection{Korrelation von prozentualer Fluoreszenzabnahme und WS Area}

- Es wurde eine signifikante, negative Korrelation zwischen der prozentualen Fluoreszenzabnahme und der WS Area festgestellt.

\subsection{Abbildung zu den Messungen}

In der nachfolgenden Abbildung 5.6 sind QLF-Aufnahmen von jeweils einem Prüfkörper jeder Gruppe zu den verschiedenen Messzeitpunkten zur Veranschaulichung dargestellt.
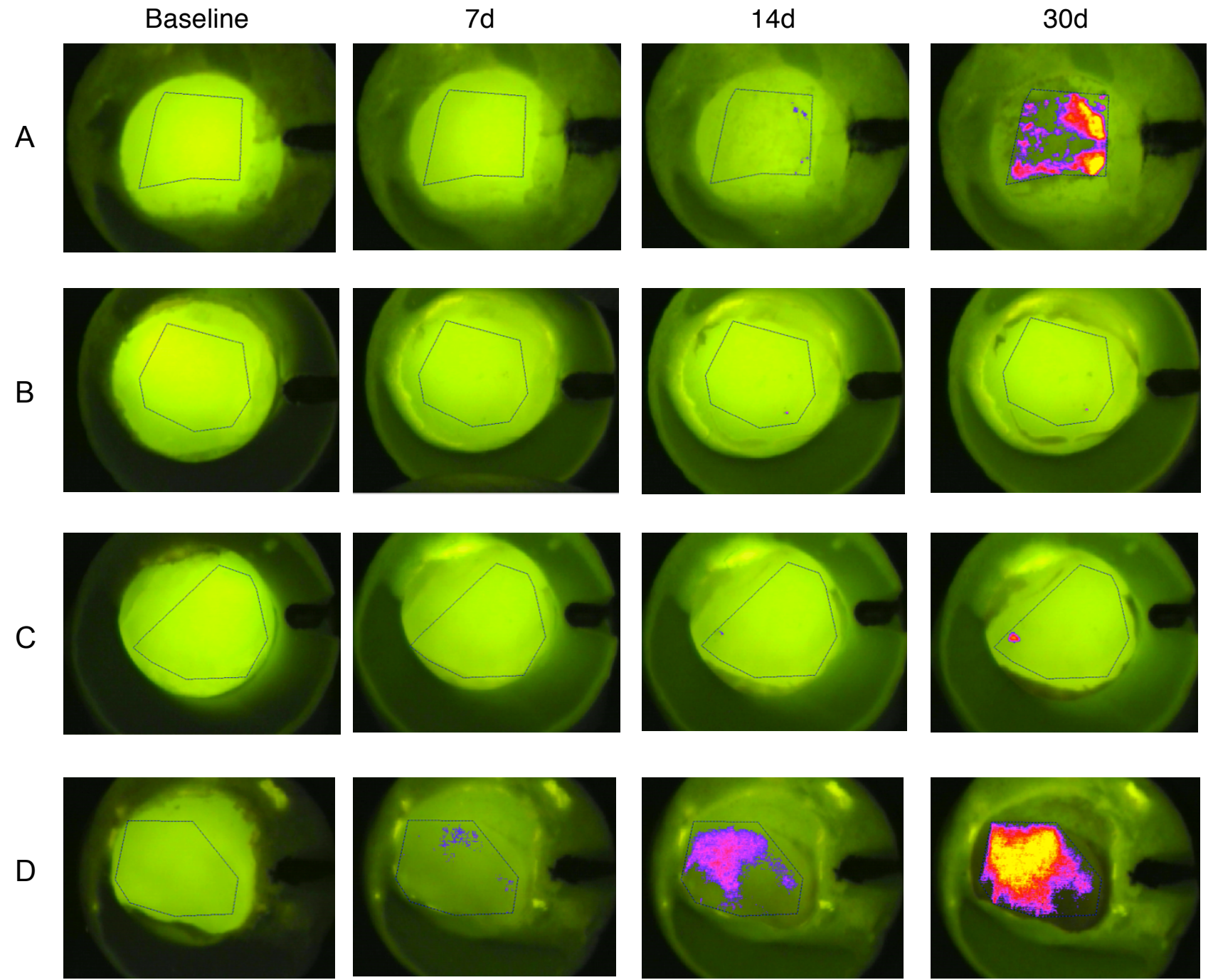

Abb.5.6 QLF- Bilder zu den verschiedenen Messzeitpunkten

(A: Cervitec Plus ${ }^{\circledR}$, B: Maximum Cure ${ }^{\circledR}$, C: ProSeal ${ }^{\circledR}$, D: Positivkontrolle) 


\section{Diskussion}

\subsection{Diskussion von Material und Methodik}

\subsubsection{Beurteilung des verwendeten in- vitro- Kariesmodells}

Um die Effektivität verschiedener Lacke zur Prävention von White- Spot- Läsionen zu testen, können in- vivo- und in- vitro- Untersuchungen Anwendung finden. In- vivo-Studien erfolgen unter klinischen Bedingungen und bieten somit den Vorteil, die Patientensituation realitätsnah wiederzugeben. Demgegenüber ermöglichen in- vitro- Studien reproduzierbare Ergebnisse unter standardisierten, hochkontrollierten Bedingungen, ohne dass individuelle Einflussparameter, wie sie bei Probanden einer klinischen Studie oft unvermeidbar sind, zu unterschiedlichen Ausgangsbedingungen führen (Sissons 1997, Seemann et al. 2005). Bei der vorliegenden in- vitro- Studie war es wichtig, die intraoralen Verhältnisse am Patienten und die klinischen Arbeitsabläufe bei Verwendung der präventiven Produkte zu simulieren. Aus diesem Grund erfolgte die Produktapplikation entsprechend ihrer Anwendung am Patienten. Des Weiteren wurde ein Kariesmodell angewendet, das trotz Vereinfachung die Verhältnisse des menschlichen Mundhöhlenmilieus und die Grundaspekte der Kariesentstehung weitgehend wiedergibt.

Im Sinne eines $\mathrm{pH}$-cyclings wurden die Zahnhartsubstanzprüfkörper alternierend De- und Remineralisationszyklen ausgesetzt, entsprechend den wechselnden $\mathrm{pH}$ - Werten mit Abfall nach Nahrungsaufnahme und Anstieg des pH- Wertes zwischen den Mahlzeiten beim Menschen (Stephan 1940). In ähnlichen Studien wurden die Prüfkörper oftmals nur einem Demineralisationsmedium ausgesetzt, eine Lagerung in einer Remineralisationslösung unterblieb (Salar et al. 2007, Buren et al. 2008, Tanna et al. 2009). Da dadurch jedoch die in vivo stattfindenden Remineralisationsprozesse durch den Speichel unberücksichtigt bleiben und somit unter Umständen eine ausgeprägtere Läsionsentstehung erzielt wird, wurde in der vorliegenden Studie das $\mathrm{pH}$ - cycling einer ausschließlichen Demineralisation vorgezogen. Auch in anderen in- vitro- Studien über WSL- Präventionsmaßnahmen wurden durch $\mathrm{pH}$ - cycling Demineralisationen erzeugt (Demito et al. 2004, Hu und Featherstone 2005, Behnan et al. 2010). Vorwiegend wurden bei diesen Studien chemische Systeme verwendet, das bedeutet, während der Demineralisation wurden Medien mit organischen Säuren, jedoch ohne Mikroorganismen eingesetzt. Somit wurden Lösungsvorgänge vollzogen, die Proteindestruktion durch Mikroorganismen blieb unberücksichtigt (Schiffner und Schmeiser 1995). In diesem Kontext gaben Buzalaf et al. (2010) jedoch zu bedenken, dass die Proteindestruktion vorwiegend bei Untersuchungen am Dentin von Bedeutung ist. Aufgrund des geringen Gehaltes an 
organischer Substanz im Schmelz beschrieben sie dessen Demineralisationsprozess als einen in erster Linie chemischen Vorgang. Durch chemische Modelle kann jedoch die Effektivität antimikrobieller Maßnahmen nicht getestet werden (Schiffner und Schmeiser 1995). Insgesamt betrachtet ermöglicht die Verwendung biologischer Systeme eine Kariesforschung mit höherer klinischer Relevanz (Fontana et al. 1996). Um auch antimikrobielle Präventionsmaßnahmen testen zu können und den intraoralen Demineralisationsprozess naturgetreuer zu simulieren, wurde deshalb in der vorliegenden Studie ein biologisches Modell eingesetzt. Es erfolgte dreimal täglich eine jeweils einstündige Demineralisation, dazwischen und über Nacht lagerten die Prüfkörper in der Remineralisationslösung. Auch ten Cate et al. (2006) und Takeshita et al. (2011) lagerten die Prüfkörper über Nacht in dem Remineralisationsmedium, führten jedoch im Tagesverlauf sechs Demineralisationszyklen durch. Somit vollzogen sie möglicherweise eine detailgenauere Simulation der intraoralen $\mathrm{pH}$ - Wert- Veränderungen, da auch Zwischenmahlzeiten berücksichtigt wurden. Im Gegensatz dazu wurde in anderen Studien ein $\mathrm{pH}$ - cycling mit weniger als drei täglichen Demineralisationsphasen durchgeführt (Hu und Featherstone 2005, Behnan et al. 2010, Karlinsey et al. 2011). Die Frequenz von (Zwischen- )Mahlzeiten und damit verbundene $\mathrm{pH}$ - Wert- Veränderungen können individuell sehr unterschiedlich sein. Da im menschlichen Tagesverlauf in der Regel zumindest drei Hauptmahlzeiten eingenommen werden, wurden in der vorliegenden Studie drei Demineralisationsphasen vollzogen.

Während dieser Demineralisationszyklen wurden die Zahnhartsubstanzprüfkörper einer Bakteriensuspension mit einem kariogenen S. mutans- Stamm ausgesetzt. S. mutans wurde gewählt, weil dieses Bakterium als Leitkeim der Kariesentstehung angesehen wird und sich seine Verwendung bereits in ähnlichen in- vitro- Studien bewährt hat (Schiffner und Schmeiser 1995, Fontana et al. 1996, Deng und ten Cate 2004). Die verwendeten Mikroorganismen wurden in Monokultur eingesetzt. Es konnte gezeigt werden, dass Bakterien, die in einer Mischflora beziehungsweise einem komplexen Biofilm existent sind, kariogener und resistenter sind als Bakterien in Monokultur (Marsh PD und Bowden 1995, Shapiro et al. 2002). Demgegenüber ist bei Verwendung verschiedener Bakterien das Feststellen einer möglichen Fremdkontamination und die Zuordnung der Demineralisation zu einem bestimmten Keim erschwert (Schiffner und Schmeiser 1995). Aufgrund dieser Problematiken wurde daher in der vorliegenden wie auch in anderen Studien eine Monokultur anstelle einer Mischflora eingesetzt (Schiffner und Schmeiser 1995, Deng und ten Cate 2004).

Die verwendete Bakteriensuspension wurde 23 Stunden nach dem Ansetzen und damit in der stationären Phase des Bakterienwachstums eingesetzt. Es ist zu diskutieren, weshalb dieser Zeitpunkt und nicht die exponentielle Phase gewählt wurde. Durch Erstellen einer Wachstumskurve konnte gezeigt werden, dass sich die optische Dichte und damit die Bakte- 
rienkonzentration während der exponentiellen Phase kontinuierlich veränderte. Demgegenüber blieb die optische Dichte während der Plateauphase über mehrere Stunden relativ konstant. Um eine annähernd gleiche Bakterienkonzentration während der Demineralisationsphasen eines Versuchstages sicherzustellen, wurde deshalb die stationäre Phase gewählt. Auch Fontana et al. (1996) nutzten Bakterienkulturen, die sich in der stationären Wachstumsphase befanden.

Der Mittelwert der täglich nach 23 Stunden bei 600nm gemessenen optischen Dichte lag bei 0,897. In der Literatur konnten keine Empfehlungen gefunden werden, in welcher Konzentration Bakterien in einem Kariesmodell anzuwenden sind, und in vielen Studien über Kariesmodelle wurde die eingesetzte Bakterienkonzentration nicht genannt (Deng und ten Cate 2004, Hess et al. 2011, Pinar Erdem et al. 2012). Guggenheim et al. (2001) und Arnold et al. (2001) setzten Bakteriensuspensionen mit einer optischen Dichte von $1.0 \pm 0.05$ (bei 550nm gemessen) beziehungsweise $1.5 \pm 0.1$ (bei 540nm gemessen) ein. Die unterschiedlich gewählten Lichtintensitäten bei den Messungen sowie die Verwendung anderer Bakterien schließen jedoch einen direkten Vergleich mit der in der vorliegenden Studie eingesetzten Bakterienkonzentration aus. Es stellt sich die Frage, ob die gewählte Bakterienkonzentration möglicherweise ein zu stark kariogenes Milieu erzeugt hat. Der pH- Wert der Demineralisationsmedien, die in verschiedenen chemischen $\mathrm{pH}$ - cycling-Modellen angewendet wurden, lag zwischen 4,3 und 5,0 (ten Cate und Duijsters 1982, Demito et al. 2004, Hu und Featherstone 2005, Karlinsey et al. 2011, Takeshita et al. 2011). Der in der vorliegenden Studie nach 23 Stunden gemessene $\mathrm{pH}$ - Wert von 4,5 lag ebenfalls in diesem Bereich. Es ist jedoch zu berücksichtigen, dass Versuchsbedingungen chemischer Systeme nur bedingt mit den Versuchsbedingungen des eingesetzten biologischen Kariesmodells verglichen werden können. Auch die Stephan- Kurve zeigt, dass bei Patienten mit erhöhter Kariesaktivität ein $\mathrm{pH}$ - Wert unter 5,0 nach Nahrungsaufnahme erreicht und damit der für den Schmelz kritische $\mathrm{pH}$ - Wert von 5,2-5,7 deutlich unterschritten wird (Stephan 1940). Gerade bei Patienten mit Multibandapparatur besteht oftmals eine erhöhte Kariesaktivität mit einem Anstieg an S.mutans und Laktobazillen sowie einem Abfall des $\mathrm{pH}$ - Wertes (Balenseifen und Madonia 1970, Buzalaf et al. 2010, Topaloglu-Ak et al. 2011). Die Stephan- Kurve zeigt jedoch weiterhin, dass es nach der Nahrungsaufnahme zu einem unmittelbaren Wiederanstieg des $\mathrm{pH}$ Wertes kommt. Auch wenn der Ausgangswert erst nach 30 bis 60 Minuten erreicht wird, liegt nur für einen kurzen Zeitraum ein pH- Wert unter 5,0 vor. Dieser Aspekt wurde in der vorliegenden Studie nicht berücksichtigt. Die Prüfkörper waren während der Demineralisationsphasen 60 Minuten durchgehend einem pH- Wert von 4,5 ausgesetzt. Auch dieser Sachverhalt deutet darauf hin, dass möglicherweise ausgeprägtere Demineralisationen als in vivo erzeugt wurden. 
Während der Remineralisationsphasen erfolgte eine Lagerung der Prüfkörper in künstlichem Speichel. Der verwendete künstliche Speichel enthält vorwiegend Mineralsalze, Hydrogenphosphat als Pufferkomponente, besitzt einen $\mathrm{pH}$ - Wert von 7,5 und verfügt somit über eine dem natürlichen Speichel vergleichbare Remineralisationswirkung. Dennoch ist zu berücksichtigen, dass künstlicher Speichel dem natürlichen Speichel ähnelt, mit diesem jedoch nicht völlig identisch ist. So fehlen in dem eingesetzten künstlichen Speichel Muzine und verschiedene Speichelenzyme. Auch in vergleichbaren Studien wurde künstlicher Speichel als Remineralisationslösung eingesetzt (Demito et al. 2004, Vieira et al. 2005, Behnan et al. 2010). Anstelle des künstlichen Speichels hätte wie in anderen Studien zuvor von Probanden entnommener Speichel verwendet werden können (Arnold et al. 2001, Guggenheim et al. 2001). Dieser ermöglicht eine detailgenauere Simulation der intraoralen Bedingungen. Jedoch variiert die Speichelzusammensetzung individuell, sodass die Verwendung von natürlichem Speichel zu variierenden Versuchsbedingungen führen könnte.

Insgesamt betrachtet stellt das verwendete Kariesmodell eine starke Vereinfachung des komplex zusammengesetzten Mundhöhlenmilieus dar. In vielen Studien werden dagegen komplexe Kariesmodelle eingesetzt, um das intraorale Milieu detailgenauer simulieren zu können (Tang et al. 2003, Seemann et al. 2006, Hayati et al. 2011). Es handelt sich zumeist um technisch aufwändige und teils computergesteuerte Apparaturen, die eine kontrollierte, kontinuierliche oder intermittierende Zufuhr unterschiedlicher Medien und die Kontrolle verschiedener Parameter, wie die Temperatur- und Gasregulation, unter sterilen Bedingungen ermöglichen. Durch Verwendung verschiedener Bakterienstämme sowie durch detailgenaue Simulation der intraoralen Umweltbedingungen wird die Bildung plaqueähnlicher Strukturen und komplexer Biofilme ermöglicht. Es ist jedoch zu bedenken, dass diese Modelle mit einem großen technischen Aufwand, höherer Kostenintensität und einer intensiveren Einarbeitung im Umgang mit diesen Apparaturen verbunden sind. Darüber hinaus stellt sich die Frage, ob diese komplexen Modelle zur Untersuchung der Grundaspekte der Karies zwingend erforderlich sind. Das Ziel der vorliegenden Studie bestand nicht darin, detailgenaue Wechselwirkungen und Charakteristika des oralen Biofilms zu evaluieren, sondern vielmehr eine Demineralisationserzeugung durch kariogene Mikroorganismen zu vollziehen, die der klinischen Situation ähnlich ist. Im Gegensatz zu den komplexen Modellen kennzeichnet sich das verwendete Kariesmodell durch die einfache Versuchsanordnung ohne aufwändige technische Apparaturen, ermöglicht somit eine praktikable Anwendung und ist kostengünstiger. Auch ohne einen komplexen Aufbau konnten weitgehend kontrollierte Versuchsbedingungen während der gesamten Studie realisiert werden. Durch tägliches Ausstreichen einer Kontrolle der verwendeten Bakteriensuspension auf COS- Platten wurde eine Fremdkontamination ausgeschlossen, durch Lagerung der Bakteriensuspension in einem Brutraum beziehungsweise in 
einem Schüttler mit Inkubationshaube wurden konstante Temperaturbedingungen hergestellt, und durch Verwendung von Anaerobierpads wurde der Gashaushalt reguliert.

Auch in anderen Studien wurde diese einfache Form des Kariesmodells gewählt und eine Bakteriensuspension als Demineralisationsmedium eingesetzt, es erfolgte jedoch kein $\mathrm{pH}$ cycling (Dummer et al. 1982, Hess et al. 2011, Pinar Erdem et al. 2012). Das in der vorliegenden Studie verwendete Kariesmodell ermöglichte demgegenüber eine Berücksichtigung der $\mathrm{pH}$ - Wert- Veränderungen im menschlichen Tagesverlauf und damit eine naturgetreuere Demineralisationserzeugung.

\subsubsection{Bewertung der QLF zur Diagnostik in vitro}

Zur Evaluation von Demineralisationserscheinungen in vitro können verschiedene diagnostische Verfahren eingesetzt werden. Die in der vorliegenden Studie angewendete quantitative lichtinduzierte Fluoreszenz (QLF) ist ein wissenschaftlich anerkanntes nicht- invasives Diagnostikverfahren, dessen Funktionsprinzip auf der unterschiedlichen Fluoreszenz von gesunder und demineralisierter Zahnhartsubstanz beruht (Ifland et al. 2004).

Die QLF wurde bereits in früheren Studien sowohl für die in- vitro- als auch in- vivoDemineralisationsdiagnostik eingesetzt (de Josselin de Jong et al. 1995, Pretty et al. 2003, Gmur et al. 2006, Nakata et al. 2009). Gmur et al. schlussfolgerten aus ihrer in- vitro- Studie, dass das getestete QLF- System geeignet ist, um eine Analyse Biofilm- induzierter De- und Remineralisationen an Schmelzprüfkörpern durchzuführen. Sie führten weiterhin die Hauptvorteile der QLF auf. Diese bestehen in der hohen Sensitivität, der Ortsspezifizität, der schnellen Durchführbarkeit und der Reproduzierbarkeit. Des Weiteren ermöglicht das QLFVerfahren die Durchführung longitudinaler Studien, bei denen mehrere Messungen an einem Objekt durchgeführt werden können, da keine Destruktion der Untersuchungsobjekte erforderlich ist. So lassen sich zeitliche Entwicklungen und Verlaufskontrollen vollziehen (Gmur et al. 2006). Auch Pretty et al. (2003) konnten in ihrer in- vitro-Studie feststellen, dass das QLFVerfahren geeignet ist, um Demineralisationserscheinungen im Bracketumfeld frühzeitig zu detektieren und im zeitlichen Verlauf zu überwachen. Dennoch ist zu erwähnen, dass das QLF-System eine sehr techniksensitive Diagnostikmethode darstellt, die durch verschiedene Faktoren negativ beeinflusst werden kann. Pretty et al. (2004) belegten, dass das Ausmaß der Hydratation der Prüfkörper die QLF- Analyse beeinflusst und eine Trocknung der Untersuchungsobjekte vor der Messung erforderlich ist. Des Weiteren kann es zu falschen Messergebnissen kommen, wenn der Winkel zwischen Kamera und Zahnoberfläche um mehr als 
$20^{\circ}$ von der Senkrechten abweicht oder der Fokusabstand nicht korrekt eingestellt wird (Ando et al. 2004, Pretty et al. 2006). Auch die Lichtintensität muss optimal eingestellt werden (Pretty et al. 2002). Aufgrund der hohen Techniksensitivität wurden in der vorliegenden Studie standardisierte Messbedingungen hergestellt, indem das Umgebungslicht während der Messungen angepasst wurde, eine vorherige Trocknung der Prüfkörper erfolgte und durch Verwendung des Video- Repositionierungssystems ein kontrollierter und während nachfolgender Messungen gleichbleibender Abstand zwischen Objekt und Kamera sichergestellt wurde. Die Positionierung der Prüfkörper auf dem Objekttisch wurde so gewählt, dass der Winkel zwischen Kamera und Prüfkörperoberfläche nicht mehr als $20^{\circ}$ von der Senkrechten abwich. Dennoch kann nicht ausgeschlossen werden, dass die Messbedingungen leicht variierten und zumindest geringen Einfluss auf die Ergebnisse nahmen.

Alternativ zu dem QLF- System hätten andere in- vitro- Diagnostikverfahren angewendet werden können. So wurde in ähnlichen in- vitro- Studien oftmals die polarisierte Lichtmikroskopie eingesetzt, um Läsionstiefen zu erfassen (Demito et al. 2004, Salar et al. 2007, Buren et al. 2008). Eine weitere alternative Diagnostikmethode stellt die transversale Mikroradiographie dar. Die TMR wird als Goldstandard der in- vitro- Kariesdiagnostik angesehen und wurde bereits in vielen Studien angewendet (Ogaard et al. 1996, Gmur et al. 2006, Takeshita et al. 2011, Lippert et al. 2012). Beide Verfahren erfordern jedoch die Destruktion der Prüfkörper und ermöglichen somit keine longitudinalen Studienverläufe wie bei der vorliegenden Untersuchung. Nach der letzten QLF- Messung hätten jedoch zusätzlich Auswertungen mit Hilfe der TMR oder der polarisierten Lichtmikroskopie durchgeführt werden können, um die Validität der QLF- Ergebnisse zu überprüfen. Gmur et al. (2006) konnten zeigen, dass bei Verwendung von der TMR und der QLF eine große Übereinstimmung zwischen beiden Diagnostikmethoden bestand, und auch Wu et al. (2010) stellten eine hohe Korrelation von mit der QLF bestimmter prozentualer Fluoreszenzabnahme und der mit dem Polarisationsmikroskop untersuchten Läsionstiefe fest. Aufgrund der belegten Übereinstimmung von QLF und TMR respektive QLF und Polarisationsmikroskopie wurde daher in der vorliegenden Studie auf den zusätzlichen Einsatz dieser Diagnostikverfahren neben der QLF verzichtet. 


\subsubsection{Auswahl und Anzahl der Zähne}

Im Rahmen der Studie wurden menschliche karies- und füllungsfreie Molaren der zweiten Dentition verwendet. Die meistverwendeten humanen Zähne in in-vitro- und in- situ- Studien sind bleibende Molaren und Prämolaren (Buzalaf et al. 2010). Aufgrund des bewährten Einsatzes wurden daher auch für die vorliegende Studie Molaren eingesetzt.

Die Verfügbarkeit menschlicher Zähne in ausreichender Qualität ist häufig limitiert, viele Zähne weisen kariöse Defekte auf, waren unterschiedlichen Umweltbedingungen (Ernährung, Fluoridzufuhr) ausgesetzt und besitzen ein unterschiedliches Alter, eine variable Herkunft und dadurch eine variablere Zusammensetzung (Mellberg 1992). In vielen Studien wurden daher bovine anstelle humaner Zähne verwendet (Attin et al. 1995, Demito et al. 2004, Gmur et al. 2006, Takeshita et al. 2011). Im Gegensatz zu humanen Zähnen ist die Verfügbarkeit und die Qualität boviner Zähne weniger problematisch und die Zusammensetzung weniger variabel (Mellberg 1992). Bei Verwendung boviner Zähne ist jedoch zu berücksichtigen, dass, auch wenn der Mineralgehalt und die Läsionsstrukturen ähnlich sind, dennoch einige Unterschiede im Vergleich zu humanen Zähnen bestehen (Edmunds et al. 1988). Boviner Schmelz verfügt über eine höhere Radioopazität, eine größere Menge an interprismatischer Substanz, und Rinderzähne weisen im Vergleich zu humanen Zähnen eine höhere Porosität auf, sodass es zu einer schnelleren Läsionsentstehung kommen kann (Edmunds et al. 1988, Mellberg 1992, Fonseca et al. 2008, Tanaka et al. 2008). Yassen et al. (2011) erstellten eine Literaturübersicht von Studien, die die Verwendung boviner und humaner Zähne verglichen. Auch sie schlussfolgerten aus ihrer Recherche, dass Unterschiede bezüglich der Morphologie, der chemischen Zusammensetzung und den physikalischen Eigenschaften berücksichtigt werden müssen, wenn Ergebnisse einer Studie mit bovinen Zähnen interpretiert werden. Aufgrund der grundlegend übereinstimmenden Reaktion von bovinen und humanen Zahnhartsubstanzen auf kariogene Bedingungen und kariespräventive Maßnahmen, können bovine Zähne aber prinzipiell alternativ verwendet werden. Dennoch stellen humane Hartsubstanzen das Substrat der ersten Wahl dar, da sie eine naturgetreuere Simulation der klinischen Situation ermöglichen (Mellberg 1992).

Um klinisch relevante Effekte statistisch zu erfassen, ist neben einer geeigneten Wahl des Prüfkörpersubstrates auch eine ausreichende Prüfkörperanzahl erforderlich. In vergleichbaren in- vitro- Studien variierte die Anzahl zwischen 10 und 20 Prüfkörpern pro Gruppe (Demito et al. 2004, Hu und Featherstone 2005, Cain et al. 2006, Salar et al. 2007). Daher wurden in der vorliegenden Studie bei den Produktgruppen sowie bei der Positivkontrollgruppe jeweils 15 Prüfkörper eingesetzt. 


\subsubsection{Verwendete Testprodukte}

Im Rahmen der Studie wurden drei verschiedene Lacke eingesetzt. Die Testlacke unterscheiden sich in ihrer Zusammensetzung, ihrem kariesprophylaktischen Wirkstoff beziehungsweise dessen Konzentration und ihrem Applikationsverfahren. Alle Lacke wurden einmalig zu Beginn der Versuchsphase appliziert.

Cervitec Plus ${ }^{\circledR}$ ist ein farbloser Lack, der auf die gereinigte und getrocknete Zahnoberfläche aufgetragen wird. Eine Konditionierung des Schmelzes mittels Säureätztechnik ist nicht erforderlich. Der Lack enthält eine Wirkstoffkombination aus Chlorhexidin (1\%) und Thymol (1\%), Fluorid ist nicht enthalten. Der Wirkstoff Thymol ist Bestandteil eines ätherischen Öls, das aus der Pflanze Thymius vulgaris gewonnen wird und wie $\mathrm{CHX}$ über eine antimikrobielle Wirksamkeit verfügt (Basch et al. 2004).

Bei den getesteten Lacken Pro Seal ${ }^{\circledR}$ und Maximum Cure ${ }^{\circledR}$ handelt es sich um fluoridhaltige Versiegler (Sealer), die im Rahmen der Bracketadhäsivtechnik angewendet werden können, um eine Demineralisation im Bereich des Brackets zu verhindern, die Haftkraft des Bondings zu steigern und versehentlich angeätzte Bereiche zu versiegeln (Hu und Featherstone 2005). Sie können vor oder nach dem Kleben der Brackets auf die Schmelzoberfläche appliziert werden. Im Rahmen der Studie erfolgte entsprechend den Herstellerangaben vor der Applikation eine Schmelzkonditionierung durch Phosphorsäure.

Maximum Cure ${ }^{\circledR}$ ist ein ungefüllter, chemisch härtender Sealer und kann nach Herstellerangaben im Rahmen der Bracketadhäsivtechnik mit verschiedenen Befestigungsmaterialien kombiniert werden. Pro Seal® ist ein lichthärtender Sealer mit hohem Füllstoffgehalt, der nach Herstellerangaben mit jedem Befestigungsmaterial angewendet werden kann. Pro Seal® enthält zudem eine Wirkstoffkomponente, die bei entsprechender Belichtung fluoresziert, sodass überprüft werden kann, ob eine gleichmäßige Beschichtung bei der Applikation erreicht wurde.

Bei der Aushärtung von Sealern kann durch Ausbildung einer Sauerstoffinhibitionsschicht eine unvollständige Polymerisation resultieren. Diese führt zu einer dünneren Sealerschicht und in der Folge zu einer schnelleren Abrasion (Buren et al. 2008). Es wird daher diskutiert, ob lichthärtende Sealer den chemisch härtenden überlegen sind, da sie einen höheren Polymerisationsgrad und somit eine vollständigere und stabilere Bedeckung der Schmelzoberfläche aufweisen (Joseph et al. 1994). Auch der Füllstoffanteil von Sealern nimmt Einfluss auf ihre Haftfestigkeit, ihre Abrasionsresistenz und damit verbunden auf ihren dauerhaften Demineralisationsschutz. Versiegelungslacke mit einem höheren Füllstoffgehalt verfügen über eine größere Abrasionsresistenz ( $\mathrm{Hu}$ und Featherstone 2005, Buren et al. 2008, Benham et al. 2009). Ein erhöhter Zusatz von Füllstoffkomponenten resultiert jedoch in einer 
erhöhten Oberflächenrauigkeit, die wiederum eine Plaqueakkumulation fördert (Banks und Richmond 1994).

Die Herstellerfirma Reliance Orthodontic Products verspricht bei Pro Seal® eine vollständige Polymerisation und auch bei Maximum Cure® durch den Zusatz von flüchtigen Lösungsmitteln eine verbesserte Aushärtung. Eine Studie von Coreil et al. (1990) zeigte jedoch, dass durch die neuen Zusätze von Maximum Cure® kein klinisch signifikanter Effekt erzielt wurde.

\subsection{Diskussion der Ergebnisse}

Das Ziel der vorliegenden Studie bestand darin, die Effektivität von drei verschiedenen Lacken zur Prävention von White- Spot- Läsionen in vitro zu testen und zu vergleichen. Verschiedene Produkte, die im Rahmen der WSL- Prävention eingesetzt werden können, wurden bereits in einigen in- vitro- Studien untersucht (Demito et al. 2004, Hu und Featherstone 2005, Cain et al. 2006, Buren et al. 2008, Behnan et al. 2010). Es existieren jedoch nur wenige Studien, die im Rahmen der Effektivitätsbeurteilung dieser Produkte ein biologisches Modell zur Erzeugung von Demineralisationen eingesetzt haben.

Bei der Negativgruppe wurden keine Produkte appliziert und die Prüfkörper wurden während der Demineralisationsphasen in einer Glucose- Bouillon ohne S. mutans gelagert. Während der gesamten Versuchsphase konnten keine Abnahmen der prozentualen Fluoreszenz oder Zunahmen der WS Area bei dieser Gruppe festgestellt werden. Bei der Positivkontrolle erfolgten ebenfalls keine Produktapplikationen, die Prüfkörper wurden jedoch während der Demineralisationsphasen einer Glucose- Bouillon mit S. mutans ausgesetzt. Die Prüfkörper dieser Gruppe wiesen die deutlichsten Demineralisationserscheinungen aller Gruppen auf. Bereits nach 7 Tagen konnte eine deutliche Abnahme der prozentualen Fluoreszenz und nach 14 Tagen eine deutliche Zunahme der WS Area festgestellt werden. Im weiteren Verlauf ließ sich eine weitere kontinuierliche Veränderung beider Parameter ermitteln. Die Kontrollgruppen zeigen, dass mit dem eingesetzten biologischen Kariesmodell eine Demineralisationserzeugung ermöglicht wurde. Die von S. mutans produzierten Säuren führten zu deutlich diagnostizierbaren Läsionen, ohne den Einsatz von S. mutans erfolgte hingegen keine Demineralisationserzeugung. Die Ergebnisse der Positivkontrolle zeigen weiterhin in Übereinstimmung mit Ergebnissen klinischer Studien, dass es ohne Demineralisationsschutz bereits nach vier Wochen zu einer deutlichen Manifestation von Schmelzläsionen kommen kann (O'Reilly und Featherstone 1987, Ogaard et al. 1988a).

Im Rahmen der Studie wurde der chlorhexidin- und thymolhaltige Lack Cervitec Plus ${ }^{\circledR}$ getestet. Chlorhexidinhaltige Lacke werden mit dem Ziel eingesetzt, durch Beeinflussung des Bak- 
terienmetabolismus sowie durch eine Reduktion der S. mutans- Zahlen das kariogene Milieu und damit das Demineralisationsrisiko zu senken (Emilson 1994). Während einige Studien einen tendenziell demineralisationshemmenden Effekt durch den ergänzenden Gebrauch von Cervitec Plus ${ }^{\circledR}$ belegen, konnte in anderen Studien kein effektiver Beitrag des Lackes zur Kariesprävention festgestellt werden (Twetman et al. 1995, Ogaard et al. 1997, Madlena et al. 2000). Auch in der vorliegenden Studie wurde kein effektiver Demineralisationsschutz durch Cervitec Plus ${ }^{\circledR}$ festgestellt. Nach 14 Tagen zeigten sich erstmals Abnahmen der prozentualen Fluoreszenz, und nach 30 Tagen bestanden keine signifikanten Unterschiede im Vergleich zu der Positivkontrolle, deren Prüfkörper schutzlos der Demineralisationslösung ausgesetzt waren. Aufgrund der Herstellerempfehlung, den Lack vierteljährlich anzuwenden, wurde Cervitec Plus ${ }^{\circledR}$ im Rahmen der vorliegenden 30- tägigen Studie nur einmalig zu Beginn appliziert. Möglicherweise sind deutlich kürzere Applikationsintervalle erforderlich, um einen dauerhaften Schutz zu gewährleisten. Auch in der Literatur wurde die Applikationsfrequenz kontrovers diskutiert. Während Madlena et al. (2000) zeigten, dass eine Applikation des Cervitec $\AA_{-}$- Lackes in einem Abstand von drei Monaten zu einer effektiven Reduzierung der S. mutans- Zahlen im Bracketumfeldbereich führen kann, empfahlen George et al. (2010) die einmonatige Applikation einem dreimonatigen Applikationsintervall vorzuziehen. Twetman et al. (1995) empfahlen eine zweimonatige Applikationsfrequenz, um eine signifikante Reduktion von S. mutans zu erreichen. Im Gegensatz zu den Studien von Madlena et al. und George et al. wurde in der vorliegenden Studie jedoch nicht die Keimzahlreduktion, sondern die grundsätzliche Schutzlackwirkung, der Einfluss auf die Bakterienadhäsion sowie die Beeinflussung des mikrobiellen Stoffwechsels durch Cervitec Plus ${ }^{\circledR}$ untersucht.

Bei den getesteten Versiegelungslacken Maximum $C u r e \AA$ und Pro Seal ${ }^{\circledR}$ zeigten sich nach 30 Tagen keine signifikanten Änderungen der untersuchten Parameter $\Delta \mathrm{F}$ und WS Area, und beide Produkte unterschieden sich signifikant von der Positivkontrolle. Auffällig war, dass bei Pro Seal ${ }^{\circledR}$ nach 14 Tagen eine Abnahme der prozentualen Fluoreszenz ermittelt wurde, diese jedoch nach 30 Tagen nicht mehr vorlag. Diese Ergebnisse könnten auf Fehlmessungen zurückzuführen sein. Möglicherweise hat die fluoreszierende Wirkstoffkomponente von Pro Seal® die QLF- Messungen beeinflusst. Die Ergebnisse nach 30 Tagen führen zu der Vermutung, dass beide Produkte die Entstehung und das Ausmaß von Demineralisationen effektiv verhindern konnten. Auch in anderen Studien wurde gezeigt, dass die beiden Versiegelungslacke das Auftreten und das Ausmaß von Demineralisationen effektiv beeinflussen können (Banks und Richmond 1994, Hu und Featherstone 2005, Cain et al. 2006, Buren et al. 2008). Zumeist wurde der Einsatz von Pro Seal® getestet, es konnten nur wenige Studien über die Effektivitätsbeurteilung von Maximum Cure ${ }^{\circledR}$ gefunden werden. So stellten $\mathrm{Hu}$ und Featherstone und Buren et al. in ihren Studien fest, dass Pro Seal ${ }^{\circledR}$ in vitro zu einer signifi- 
kanten Verringerung der Schmelzdemineralisation im Vergleich zu der Kontrollgruppe, zu einem Fluoridlack und einem ungefüllten Sealer führte. In einer Studie von Banks und Richmond (1994) zeigte sich eine signifikante, jedoch geringe Reduktion in der Inzidenz und dem Ausmaß von Demineralisationen nach Verwendung von Maximum Cure ${ }^{\circledR}$.

Einige Studien zeigen, dass fluoridfreisetzende Materialien, die im Rahmen der Bracketadhäsivtechnik eingesetzt werden, vorwiegend in den ersten 24 Stunden nach der Applikation Fluoride freisetzen und die Effektivität dieser Materialien nur für einen limitierten Zeitraum nachgewiesen werden kann (Wiltshire und Janse van Rensburg 1995, Basdra et al. 1996, McNeill et al. 2001, Cohen et al. 2003). Soliman et al. (2006) untersuchten die Fluoridfreisetzung von Pro Seal® und konnten zeigen, dass es innerhalb von 17 Wochen zu einer starken Reduzierung der Fluoridfreisetzung kam. In der Studie erfolgte jedoch keine Untersuchung der Effektivität des Sealers zur WSL- Prävention. Möglicherweise ist die nach 17 Wochen festgestellte geringe Fluoridfreisetzung dennoch ausreichend, um eine Demineralisation zu verhindern. McNeill et al. stellten fest, dass sich die Fluoridfreisetzung der getesteten Materialien zwar deutlich reduzierte, jedoch im Untersuchungszeitraum von sechs Monaten stets eine die Schmelzdekalzifikation hemmende Fluoridfreisetzung vorlag. Da der Behandlungszeitraum mit festsitzenden Apparaturen zumeist sechs Monate überschreitet, ist jedoch fraglich, ob eine kariesprotektive Wirkung während der gesamten Behandlungsdauer besteht (Pseiner 2008).

In der vorliegenden Untersuchung konnten nach 30 Tagen keine signifikanten Unterschiede hinsichtlich der Effektivität zwischen dem chemisch härtenden, ungefüllten und dem lichthärtenden, hochgefüllten Sealer festgestellt werden. Dieses Ergebnis ist konträr zu Ergebnissen anderer Studien, in denen niedriggefüllte Sealer lichthärtenden Sealern mit hohem Füllstoffgehalt aufgrund einer verringerten Abrasionsresistenz unterlegen waren $\mathrm{Hu}$ und Featherstone 2005, Buren et al. 2008). Im Rahmen der vorliegenden Studie wurden die Prüfkörper keinen Abrasionsvorgängen wie zum Beispiel dem Zähneputzen ausgesetzt. Möglicherweise kann dieser Aspekt erklären, dass kein signifikanter Unterschied zwischen beiden Produkten bestand.

Beim Vergleich der Sealer mit dem Lack Cervitec Plus ${ }^{\circledR}$ wurden keine signifikanten Unterschiede der prozentualen Fluoreszenzabnahme festgestellt. Cervitec Plus ${ }^{\circledR}$ wies jedoch tendenziell schlechtere Werte auf. Bei Untersuchung der WS Area zeigten die beiden Sealer signifikant geringere Zunahmen des Parameters. Im Gegensatz zu den beiden Sealern wird Cervitec Plus ${ }^{\circledR}$ ohne vorherige Schmelzätzung auf die gereinigte und getrocknete Zahnoberfläche appliziert. Möglicherweise besteht daher ein geringerer Verbund zwischen dem Lack und der Zahnoberfläche als bei den Sealern. Es könnten Abplatzungen des Lackes aufgetreten sein, und die resultierenden Diskontinuitäten der Lackschicht haben möglicherweise zu 
einer reduzierten Schutzwirkung geführt. Eine weitere mögliche Erklärung der deutlicheren Demineralisationen von Cervitec Plus ${ }^{\circledR}$ im Vergleich zu den Sealern könnte in einer unterschiedlichen Effektivität der kariespräventiven Inhaltsstoffe bestehen. Während die Effektivität fluoridhaltiger Produkte zur Prävention von White- Spot- Läsionen vielfach belegt worden ist (Geiger et al. 1992, Benson et al. 2004, Demito et al. 2004, Farhadian et al. 2008), wird die Effektivität $\mathrm{CHX}$ - haltiger Lacke sehr unterschiedlich bewertet. Das Hauptproblem besteht in der langfristigen Reduktion von Streptococcus mutans durch Chlorhexidin, zumeist kommt es zu einer Rekolonisierung. Des Weiteren führt eine Reduktion von Streptococcus mutans nicht immer zu einer Abnahme der Kariesprävalenz (Autio-Gold 2008). Aufgrund fehlender Evidenz chlorhexidinhaltiger Produkte werden daher oftmals fluoridhaltige Produkte als Mittel der Wahl zur Prävention von WSL angesehen (Autio-Gold 2008, James et al. 2010, Bergstrand und Twetman 2011, Keim 2011). Auch Zaura-Arite und ten Cate (2000) zeigten, dass fluoridhaltige Lacke einen größeren demineralisationshemmenden Effekt aufweisen als der Lack Cervitec ${ }^{\circledR}$. Im Gegensatz dazu stellten Petersson et al. (2000) in einer Vergleichsstudie von Cervitec ${ }^{\circledR}$ und dem fluoridhaltigen Lack Fluor Protector ${ }^{\circledR}$ fest, dass beide Produkte eine ähnliche Effektivität zur Kontrolle der Kariesinzidenz aufwiesen.

Auch wenn Fluoride einen wichtigen Beitrag in der WSL- Prävention leisten, kann bei orthodontischen Patienten mit sehr hoher Kariesaktivität oft allein durch erhöhte Zahnputzfrequenz und ausgedehnte Fluoridierungsmaßnahmen der Demineralisationsprozess nicht gestoppt werden (Ogaard et al. 1994). In diesen Fällen könnte eine kombinierte Anwendung von $\mathrm{CHX}$ zusätzlich zu Fluoridierungsmaßnahmen dazu beitragen, das Kariesrisiko zu senken. Verschiedene Studien belegen, dass die kombinierte Anwendung von $\mathrm{CHX}$ und Fluoriden effektiv dem Entstehen von Demineralisationen im Rahmen kieferorthopädischer Behandlungen entgegenwirken kann (Ogaard et al. 2001, Alves et al. 2008, Pinar Erdem et al. 2012).

Es stellt sich generell die Frage, wann und in welcher Intensität Präventionsmaßnahmen im Rahmen kieferorthopädischer Behandlungen nötig sind, da individuell starke Unterschiede hinsichtlich des Demineralisationsrisikos bestehen können (Lovrov et al. 2007, Enaia et al. 2011, Hadler-Olsen et al. 2011). So konnte bei Patienten, bei denen Indizes wie zum Beispiel der approximale Plaque Index erhöht waren, ein stärkeres Auftreten von WSL verzeichnet werden als bei Patienten mit guter Mundhygiene. In diesem Kontext schlugen Zimmer und Rottwinkel (2004) vor, bereits vor Behandlungsbeginn das individuelle Kariesrisiko anhand von Hygieneparametern zu bestimmen. Bei Patienten mit hohem Kariesrisiko sollte dann eine ausgedehntere prophylaktische Betreuung während der kieferorthopädischen Behandlung stattfinden als bei Patienten mit niedrigem Kariesrisiko. Da neben der Compliance jedoch weitere Risikofaktoren wie das Geschlecht, das Alter oder die Behandlungsdauer 
diskutiert werden, sollten auch diese Aspekte bei der Beurteilung des Demineralisationsrisikos berücksichtigt werden.

Im Rahmen der statistischen Auswertung wurde des Weiteren geprüft, inwieweit die untersuchten Parameter der prozentualen Fluoreszenzabnahme und die Zunahme der WS Area korreliert waren. In der Literatur konnten keine Studien gefunden werden, in denen eine Korrelation von prozentualer Fluoreszenz und WS Area untersucht wurde, und somit konnten keine Vergleiche der eigenen Ergebnisse mit anderen Resultaten erstellt werden.

Die Ergebnisse der vorliegenden Studie zeigten, dass eine signifikante negative, jedoch nicht lineare Korrelation vorlag. Das bedeutet, dass bei Abnahme der prozentualen Fluoreszenz zugleich eine Zunahme der WS Area ermittelt wurde. Jedoch veränderten sich die beiden Parameter nicht immer in gleichem Ausmaß. Während bei Cervitec Plus ${ }^{\circledR}$ nach 14 Tagen eine prozentuale Fluoreszenzabnahme ermittelt wurde, wies die WS Area erst nach $30 \mathrm{Ta}$ gen eine deutliche Zunahme auf. Auch bei der Positivkontrolle lag nach 7 Tagen eine deutliche Abnahme der prozentualen Fluoreszenz vor, eine Zunahme der WS Area wurde jedoch erst nach 14 Tagen erkennbar. Während bei Betrachtung der WS Area bei allen Versuchsgruppen nur geringe Messwertveränderungen im zeitlichen Verlauf auftraten, zeigten sich bei der prozentualen Fluoreszenzabnahme deutlichere Veränderungen. Möglicherweise stellt die prozentuale Fluoreszenz einen Parameter mit höherer Sensitivität dar. Es könnte sein, dass das QLF- System früher eine Änderung der prozentualen Fluoreszenz als eine Veränderung der WS Area erfasst. Im Widerspruch dazu steht jedoch die Beobachtung, dass nach 30 Tagen ein tendenzieller, jedoch nicht signifikanter Unterschied der prozentualen Fluoreszenzabnahme zwischen Cervitec Plus ${ }^{\circledR}$ und den beiden Sealern vorlag. Demgegenüber war der Unterschied bei Betrachtung der WS Area signifikant. Eine mögliche Erklärung könnte darin bestehen, dass der Parameter der prozentualen Fluoreszenz leichter durch variierende Messbedingungen beeinflusst wird. Möglicherweise bestanden zum Beispiel geringe Variationen des Umgebungslichtes bei den Messungen, die auf die prozentuale Fluoreszenz Einfluss nahmen.

\subsection{Limitationen der Studie}

Bei möglichen Schlussfolgerungen aus den Ergebnissen sind einige Limitationen der vorliegenden Studie zu bedenken. So ist zu berücksichtigen, dass die Effektivität der Testprodukte in der vorliegenden Studie in einem Zeitraum von vier Wochen untersucht wurde. Die Behandlung mit festsitzenden Apparaturen erfolgt jedoch über mehrere Monate. So stellt sich 
die Frage, inwieweit durch den Versuchszeitraum von vier Wochen beurteilt werden kann, ob die getesteten Produkte einen Langzeitschutz vor Demineralisationen gewährleisten.

Ein weiterer limitierender Faktor besteht darin, dass keine Simulation abrasiver Vorgänge erfolgte. Somit bleibt die Abrasionsresistenz der Testprodukte unberücksichtigt. Im Gegensatz dazu erfolgte in vergleichbaren Studien eine Zahnputzsimulation (Hu und Featherstone 2005, Buren et al. 2008). Durch Einsatz von Zahnputzsimulatoren können die intraoralen Abrasionsvorgänge jedoch nur bedingt simuliert werden, nahrungsbedingte Abrasionen finden weiterhin keine Berücksichtigung (Buren et al. 2008).

Auch die täglichen Mundhygienemaßnahmen des Patienten blieben ohne Verwendung des Zahnputzsimulators unberücksichtigt. Bei Interpretation der Ergebnisse muss daher bedacht werden, dass die Testprodukte klinisch zusätzlich zu den täglichen Mundhygienemaßnahmen des Patienten eingesetzt werden und die Ergebnisse unter Berücksichtigung des Aspektes, dass nicht geputzt wurde, beurteilt werden sollten. Da keine plaquereduzierenden Maßnahmen durchgeführt wurden, sind die entstandenen Läsionen möglicherweise ausgeprägter als in vivo. Tanna et al. (2009) konnten feststellen, dass eine Demineralisationsentstehung von einer mechanischen Zahnputzsimulation nicht signifikant beeinflusst wird. In ihrer Studie ließen sich keine signifikanten Unterschiede der Läsionstiefe zwischen keiner Zahnputzsimulation, zwei- oder sechsminütiger Simulation feststellen. Auch der Einfluss von (fluoridhaltiger) Zahnpasta, die in der Regel im Rahmen der täglichen Zahnputzgewohnheiten der Patienten eingesetzt wird, wurde nicht simuliert. Um jedoch das grundsätzliche kariespräventive Potential der Produkte ohne Einflüsse oder Wechselwirkungen testen zu können, erfolgten in der vorliegenden Studie wie auch in vergleichbaren Studien keine zusätzlichen Maßnahmen wie die Anwendung fluoridhaltiger Zahnpasta (Salar et al. 2007, Sudjalim et al. 2007, Karlinsey et al. 2011).

Obwohl in der vorliegenden Studie Produkte zur Prävention von WSL im Rahmen kieferorthopädischer Behandlungen getestet wurden, erfolgte keine Bracketapplikation auf die Schmelzproben. In der Literatur finden sich sowohl Studien, die Brackets verwendet haben, als auch Studien, die ebenfalls auf den Einsatz von Brackets verzichtet haben (Demito et al. 2004, Hu und Featherstone 2005, Cain et al. 2006, Buren et al. 2008, Behnan et al. 2010). Inwieweit der Einsatz von Brackets sinnvoll ist, hängt von der Fragestellung der Studie ab. Wenn getestet werden soll, inwieweit im unmittelbaren Bracketumfeld der Einsatz eines kariespräventiven Produktes effektiv ist oder ob es Unterschiede in der Schutzwirkung in bracketfernen und bracketnahen Bereichen gibt, kann die Verwendung von Brackets sinnvoll sein. Das Ziel der vorliegenden Studie bestand jedoch darin zu testen, ob durch die Produkte grundsätzlich das Entstehen und das Ausmaß von White- Spot- Läsionen beeinflusst werden können. Daher wurde auf den Einsatz von Brackets verzichtet. 
Eine weitere Limitation der Studie besteht in der Anwendung eines einfachen Kariesmodells. Dieses stellt eine starke Vereinfachung des intraoralen Mundhöhlenmilieus dar. Der intraorale komplexe Biofilm sowie verschiedene intraorale Parameter, die bei der Kariesätiologie von Bedeutung sind, wurden nicht simuliert. So erfolgte keine Simulation von Speichelfluss oder oraler Clearance, es wurde eine Monokultur verwendet, und der intraorale pH- Wert-

Wechsel wurde in vereinfachter Form vollzogen. Alternativ hätten komplexere Modelle eingesetzt werden können, die eine detailgenauere Simulation ermöglicht hätten. Da jedoch in der bestehenden Arbeitsgruppe in Göttingen noch keinerlei Erfahrungen mit biologischen Modellen vorlagen, galt es zunächst, ein einfaches Modell zu etablieren, dessen Aufbau unter den gegebenen Rahmenbedingungen umsetzbar war. Auf diesem Modell können nachfolgende Studien aufbauen, um eine detailgenauere Simulation zu erreichen. Auch Marsh PD und Bowden (1995) betonten, dass die Auswahl eines geeigneten Modells von der Fragestellung der Studie und den gegebenen Rahmenbedingungen abhängt. Einfache Kariesmodelle können die klinische Situation nur bedingt simulieren; jedoch können sie verwendet werden, um die kariespräventive Effektivität verschiedener Produkte initial zu beurteilen. Generell sind Ergebnisse von in- vitro- Studien nur limitiert auf die klinische Situation zu übertragen. Auch komplexe Kariesmodelle ermöglichen nur eine eingeschränkte Simulation der komplexen biologischen Prozesse, die bei der Kariespathogenese von Bedeutung sind. Somit bleibt die Notwendigkeit nachfolgender klinischer Studien auch bei diesen Modellen bestehen (White 1995, Tang et al. 2003, Buzalaf et al. 2010). 


\subsection{Schlussfolgerung}

Das eingesetzte Kariesmodell ermöglicht eine der klinischen Situation ähnliche Demineralisationserzeugung und stellt eine geeignete Methode dar, um Produkte mit kariespräventiver Wirksamkeit in vitro zu testen.

Die Ergebnisse der Studie zeigen, dass die fluoridhaltigen Versiegelungslacke Pro Seal® und Maximum Cure ${ }^{\circledR}$ unter den Rahmenbedingungen der Studie einen effektiven Demineralisationsschutz ermöglichten. Der chlorhexidin- und thymolhaltige Lack Cervitec Plus ${ }^{\circledR}$ wies dagegen keinen deutlichen Demineralisationsschutz auf.

Die in der Literatur beschriebene geringere Effektivität chemisch härtender ungefüllter Sealer im Vergleich zu lichthärtenden Sealern mit hohem Füllstoffgehalt konnte in der vorliegenden Studie nicht festgestellt werden.

Aufgrund der generell limitierten Übertragbarkeit der Ergebnisse von in- vitro- Studien sind weitere klinische Studien erforderlich, um die festgestellte Effektivität der fluoridhaltigen Versiegelungslacke auch in vivo zu verifizieren.

\subsection{Ausblick}

In nachfolgenden Studien kann das eingesetzte Kariesmodell weiterentwickelt werden, um eine noch naturgetreuere Simulation des intraoralen Kariesprozesses zu erreichen. Mögliche Ansätze einer Weiterentwicklung könnten darin bestehen, eine Mischflora einzusetzen, die eingesetzten Bakterienkonzentrationen zu variieren oder das $\mathrm{pH}$-cycling zu modifizieren.

Zukünftige Studien könnten zudem durch Simulation abrasiver Vorgänge Aufschlüsse darüber geben, ob Maximum Cure ${ }^{\circledR}$ aufgrund seines Härtungsmodus und dem Fehlen von Füllstoffen dem lichthärtenden, hochgefüllten Sealer Pro Seal® unterlegen ist.

Da die Bandbreite verschiedener Präventionsmaßnahmen den Behandler vor die Frage stellen, welche Maßnahmen einen optimalen Demineralisationsschutz bieten, sollten weitere Studien darauf abzielen, die verschiedenen Maßnahmen klinisch zu beurteilen und zu vergleichen. Das Ziel sollte darin bestehen, eindeutige und evidenzbasierte Empfehlungen zur Prävention von White- Spot- Läsionen im Rahmen kieferorthopädischer Behandlungen aussprechen zu können. 


\section{Zusammenfassung}

Das Ziel der vorliegenden Studie bestand darin, die Effektivität von zwei fluoridhaltigen Versiegelungslacken und einem chlorhexidin- und thymolhaltigen Lack in einem biologischen Kariesmodell in vitro zu untersuchen und zu vergleichen.

Für die Untersuchung wurden aus menschlichen kariesfreien Molaren der zweiten Dentition 65 Schmelzprüfkörper hergestellt und diese vier Versuchsgruppen randomisiert zugewiesen (A: Cervitec Plus ${ }^{\circledR}$, B: Maximum Cure ${ }^{\circledR}$, C: Pro Seal ${ }^{\circledR}$, D: Positivkontrolle, E: Negativkontrolle; bei den Gruppen A-D $n=15$, bei Gruppe $E n=5$ ). Vor Versuchsbeginn erfolgte eine Baselinemessung mittels quantitativer lichtinduzierter Fluoreszenz (QLF). Zu Beginn der Versuchsphase erfolgte die einmalige Produktapplikation nach Herstellerangaben. Im Versuchsverlauf erfolgten täglich alternierende De- und Remineralisationszyklen mit Lagerung der Prüfkörper in einer biologischen Demineralisationslösung und künstlichem Speichel als Remineralisationslösung. Über Nacht wurden die Prüfkörper ebenfalls in künstlichem Speichel gelagert. Die Läsionstiefe, ausgedrückt durch die prozentuale Fluoreszenzabnahme $(\Delta \mathrm{F}$ in $\%$ ) und die Zunahme der WS Area (in $\mathrm{mm}^{2}$ ) im Vergleich zur Baselinemessung, wurde mit dem QLF- Verfahren nach 7, 14 und 30 Tagen ermittelt; die Resultate der Messungen wurden statistisch ausgewertet. Des Weiteren wurde durch Bestimmung des Korrelationskoeffizienten nach Pearson geprüft, inwieweit die untersuchten Parameter der prozentualen Fluoreszenzabnahme und die Zunahme der WS Area korreliert waren.

Mit Ausnahme der Negativkontrolle wurden bei allen Gruppen signifikante Abnahmen der prozentualen Fluoreszenz beziehungsweise Zunahmen der WS Area festgestellt. Nach 30 Tagen wies die Positivkontrolle die deutlichste Veränderung der untersuchten Parameter auf. Unter den Testprodukten zeigte Cervitec Plus ${ }^{\circledR}$ die deutlichste Veränderung von $\Delta \mathrm{F}$ und WS Area. Maximum Cure ${ }^{\circledR}$ und Pro Seal ${ }^{\circledR}$ zeigten nach 30 Tagen keine signifikanten Veränderungen der Parameter. Bei den erstellten Gruppenvergleichen zeigte sich, dass Cervitec Plus ${ }^{\circledR}$ im Gegensatz zu Maximum Cure ${ }^{\circledR}$ und Pro Seal ${ }^{\circledR}$ nach 30 Tagen signifikante Unterschiede zu der Negativkontrolle, nicht jedoch zu der Positivkontrolle aufwies. Des Weiteren zeigte Cervitec Plus ${ }^{\circledR}$ nach 30 Tagen eine tendenziell größere Abnahme der prozentualen Fluoreszenz und signifikant größere Zunahme der WS Area als Maximum Cure ${ }^{\circledR}$ und Pro Seal®. Zwischen den Parametern prozentuale Fluoreszenzabnahme und WS Area bestand eine signifikante negative Korrelation.

Zusammenfassend zeigten die Ergebnisse, dass das verwendete Kariesmodell geeignet ist, um eine der klinischen Situation ähnliche Demineralisationserzeugung in vitro zu erzielen. Die Ergebnisse deuten zudem darauf hin, dass die getesteten fluoridhaltigen Versiegelungs- 
lacke Maximum Cure ${ }^{\circledR}$ und Pro Seal ${ }^{\circledR}$ im Gegensatz zu dem chlorhexidin- und thymolhaltigen Lack Cervitec Plus ${ }^{\circledR}$ unter den Rahmenbedingungen der Studie einen effektiven Demineralisationsschutz ermöglichten. Aufgrund der limitierten Übertragbarkeit von in- vitroStudienergebnissen auf die klinische Situation sollten weitere Studien am Patienten folgen. 


\section{Anhang}

\subsection{OD- Messwerte der eingesetzten Bakteriensuspensionen}

Tab.8.1 Messwerte der täglich 23 Stunden nach dem Ansetzen der Bakteriensuspension gemessenen optischen Dichte

\begin{tabular}{|c|c|c|}
\hline Versuchstag & $\begin{array}{c}\text { Optische Dichte } \\
\text { (gemessen bei 600nm) }\end{array}$ & $\begin{array}{c}\text { Unkontrollierte Verkeimung } \\
\text { (nach } 48 \text { h kontrolliert) }\end{array}$ \\
\hline 1 & 1,334 & Nein \\
\hline 2 & 0,734 & Nein \\
\hline 3 & 0,846 & Nein \\
\hline 4 & 1,077 & Nein \\
\hline 5 & 0,934 & Nein \\
\hline 6 & 0,854 & Nein \\
\hline 7 & 0,897 & Nein \\
\hline 8 & 0,880 & Nein \\
\hline 9 & 1.000 & Nein \\
\hline 10 & 1.053 & Nein \\
\hline 11 & 0.914 & Nein \\
\hline 12 & 1,034 & Nein \\
\hline 13 & 0,905 & Nein \\
\hline 14 & 0,843 & Nein \\
\hline 15 & 0,808 & Nein \\
\hline 16 & 0,835 & Nein \\
\hline 17 & 0,878 & Nein \\
\hline 18 & 0,780 & Nein \\
\hline 19 & 0,898 & Nein \\
\hline 20 & 0,928 & Nein \\
\hline 21 & 0,994 & Nein \\
\hline 22 & 0,820 & Nein \\
\hline 23 & 0,854 & Nein \\
\hline 24 & 1,014 & Nein \\
\hline 25 & 0,824 & Nein \\
\hline 26 & 0,783 & Nein \\
\hline 27 & 0,883 & Nein \\
\hline 28 & 0,868 & Nein \\
\hline 29 & 0,586 & Nein \\
\hline 30 & 0,856 & Nein \\
\hline
\end{tabular}




\subsection{QLF- Einzelmesswerte der Prüfkörper}

\subsubsection{Prozentuale Fluoreszenzabnahme $(\Delta F)$}

Tab.8.2 Einzelmesswerte der prozentualen Fluoreszenzabnahme zur Baseline

\begin{tabular}{|c|c|c|c|c|c|}
\hline Baseline & & & & & \\
\hline Prüfkörper & $\begin{array}{l}\text { Cervitec } \\
\text { Plus }{ }^{\circledR}\end{array}$ & $\begin{array}{l}\text { Maximum } \\
\text { Cure }{ }^{8}\end{array}$ & Pro Sealß & $\begin{array}{l}\text { Positiv- } \\
\text { kontrolle }\end{array}$ & $\begin{array}{l}\text { Negativ- } \\
\text { kontrolle }\end{array}$ \\
\hline 1 & 0 & 0 & 0 & 0 & 0 \\
\hline 2 & 0 & 0 & 0 & 0 & 0 \\
\hline 3 & 0 & 0 & 0 & 0 & 0 \\
\hline 4 & 0 & 0 & 0 & 0 & 0 \\
\hline 5 & 0 & 0 & 0 & 0 & 0 \\
\hline 6 & 0 & 0 & 0 & 0 & - \\
\hline 7 & 0 & 0 & 0 & 0 & - \\
\hline 8 & 0 & 0 & 0 & 0 & - \\
\hline 9 & 0 & 0 & 0 & 0 & - \\
\hline 10 & 0 & 0 & 0 & 0 & - \\
\hline 11 & 0 & 0 & 0 & 0 & - \\
\hline 12 & 0 & 0 & 0 & 0 & - \\
\hline 13 & 0 & 0 & 0 & 0 & - \\
\hline 14 & 0 & 0 & 0 & 0 & - \\
\hline 15 & 0 & 0 & 0 & 0 & - \\
\hline
\end{tabular}

Tab.8.3 Einzelmesswerte der prozentualen Fluoreszenzabnahme nach 7 Tagen

\begin{tabular}{|c|c|c|c|c|c|}
\hline Nach 7 Tagen & & & & & \\
\hline Prüfkörper & $\begin{array}{l}\text { Cervitec } \\
\text { Plus }{ }^{\circledR}\end{array}$ & $\begin{array}{l}\text { Maximum } \\
\text { Cure }{ }^{8}\end{array}$ & Pro Seal® & $\begin{array}{l}\text { Positiv- } \\
\text { kontrolle }\end{array}$ & $\begin{array}{l}\text { Negativ- } \\
\text { kontrolle }\end{array}$ \\
\hline 1 & 0 & $-7,66$ & $-9,37$ & $-5,87$ & 0 \\
\hline 2 & $-9,49$ & $-5,7$ & 0 & $-9,7$ & 0 \\
\hline 3 & $-5,69$ & $-7,8$ & 0 & 0 & 0 \\
\hline 4 & 0 & $-6,64$ & $-6,91$ & $-5,94$ & 0 \\
\hline 5 & 0 & 0 & $-9,9$ & 0 & 0 \\
\hline 6 & 0 & $-9,49$ & 0 & $-5,56$ & 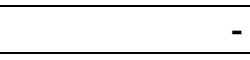 \\
\hline 7 & 0 & 0 & 0 & $-7,53$ & 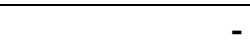 \\
\hline 8 & $-5,97$ & 0 & 0 & 0 & - \\
\hline 9 & 0 & 0 & 0 & $-5,84$ & - \\
\hline 10 & $-5,84$ & 0 & 0 & $-7,89$ & 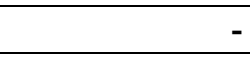 \\
\hline 11 & 0 & 0 & 0 & $-5,56$ & 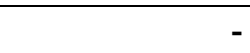 \\
\hline 12 & $-5,56$ & 0 & 0 & $-6,16$ & - \\
\hline 13 & 0 & $-7,1$ & 0 & $-6,45$ & - \\
\hline 14 & 0 & 0 & $-9,27$ & 0 & . \\
\hline 15 & 0 & $-6,72$ & 0 & 0 & - \\
\hline
\end{tabular}


Tab.8.4 Einzelmesswerte der prozentualen Fluoreszenzabnahme nach 14 Tagen

\begin{tabular}{|c|c|c|c|c|c|}
\hline Nach 14 Tagen & & & & & \\
\hline Prüfkörper & Cervitec Plus ${ }^{\circledR}$ & $\begin{array}{l}\text { Maximum } \\
\text { Cure }{ }^{\circledR}\end{array}$ & Pro Seal® & $\begin{array}{l}\text { Positiv- } \\
\text { kontrolle }\end{array}$ & $\begin{array}{l}\text { Negativ- } \\
\text { kontrolle }\end{array}$ \\
\hline 1 & $-5,66$ & $-6,22$ & $-6,78$ & $-9,01$ & 0 \\
\hline 2 & $-7,3$ & $-6,02$ & 0 & $-9,08$ & 0 \\
\hline 3 & $-7,97$ & 0 & $-11,1$ & $-7,14$ & 0 \\
\hline 4 & $-6,49$ & 0 & 0 & $-9,41$ & 0 \\
\hline 5 & 0 & 0 & $-7,65$ & $-6,8$ & 0 \\
\hline 6 & $-5,94$ & $-8,73$ & $-5,93$ & $-7,35$ & 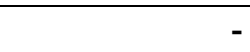 \\
\hline 7 & $-6,54$ & 0 & $-9,52$ & $-9,09$ & 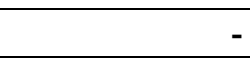 \\
\hline 8 & 0 & 0 & 0 & $-8,61$ & - \\
\hline 9 & 0 & 0 & $-7,48$ & $-6,96$ & - \\
\hline 10 & $-6,02$ & 0 & 0 & $-6,35$ & 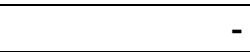 \\
\hline 11 & 0 & 0 & 0 & $-7,51$ & 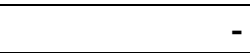 \\
\hline 12 & $-6,31$ & $-6,95$ & 0 & $-9,79$ & - \\
\hline 13 & 0 & $-7,79$ & $-6,27$ & $-8,88$ & - \\
\hline 14 & 0 & $-9,2$ & $-13,5$ & $-5,93$ & . \\
\hline 15 & $-6,28$ & $-8,37$ & 0 & $-5,9$ & - \\
\hline
\end{tabular}

Tab.8.5 Einzelmesswerte der prozentualen Fluoreszenzabnahme nach 30 Tagen

\begin{tabular}{|c|c|c|c|c|c|}
\hline Nach 30 Tagen & & & & & \\
\hline Prüfkörper & $\begin{array}{l}\text { Cervitec } \\
\text { Plus }{ }^{\circledR}\end{array}$ & $\begin{array}{l}\text { Maximum } \\
\text { Cure }{ }^{8}\end{array}$ & Pro Sealß & $\begin{array}{l}\text { Positiv- } \\
\text { kontrolle }\end{array}$ & $\begin{array}{l}\text { Negativ- } \\
\text { kontrolle }\end{array}$ \\
\hline 1 & $-6,13$ & $-7,2$ & $-8,53$ & $-19,7$ & 0 \\
\hline 2 & $-9,6$ & 0 & 0 & $-12,1$ & 0 \\
\hline 3 & $-11,6$ & 0 & $-21,9$ & $-13,8$ & 0 \\
\hline 4 & $-15,2$ & 0 & 0 & $-16,6$ & 0 \\
\hline 5 & $-8,04$ & 0 & $-7,66$ & $-11,6$ & 0 \\
\hline 6 & $-14,5$ & $-6,94$ & $-5,57$ & $-10,2$ & 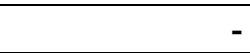 \\
\hline 7 & $-8,91$ & 0 & 0 & -17 & - \\
\hline 8 & $-8,63$ & 0 & 0 & $-19,9$ & 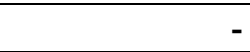 \\
\hline 9 & $-7,63$ & 0 & 0 & $-9,24$ & - \\
\hline 10 & $-8,8$ & 0 & 0 & $-8,25$ & 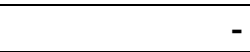 \\
\hline 11 & $-12,6$ & -11 & 0 & $-9,83$ & - \\
\hline 12 & $-15,6$ & $-16,3$ & 0 & $-20,5$ & 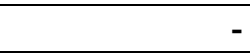 \\
\hline 13 & $-10,4$ & $-6,55$ & -15 & $-11,8$ & - \\
\hline 14 & $-5,96$ & $-11,5$ & $-13,6$ & $-7,06$ & - \\
\hline 15 & $-8,32$ & $-19,7$ & 0 & $-10,4$ & - \\
\hline
\end{tabular}




\subsubsection{WS Area}

Tab.8.6 Einzelmesswerte der Zunahme der WS Area zur Baseline

\begin{tabular}{|c|c|c|c|c|c|}
\hline Baseline & & & & & \\
\hline Prüfkörper & $\begin{array}{l}\text { Cervitec } \\
\text { Plus }{ }^{\circledR}\end{array}$ & $\begin{array}{l}\text { Maximum } \\
\text { Cure }{ }^{\circledR}\end{array}$ & Pro Seal ${ }^{\circledR}$ & $\begin{array}{l}\text { Positiv- } \\
\text { kontrolle }\end{array}$ & $\begin{array}{l}\text { Negativ- } \\
\text { kontrolle }\end{array}$ \\
\hline 1 & 0 & 0 & 0 & 0 & 0 \\
\hline 2 & 0 & 0 & 0 & 0 & 0 \\
\hline 3 & 0 & 0 & 0 & 0 & 0 \\
\hline 4 & 0 & 0 & 0 & 0 & 0 \\
\hline 5 & 0 & 0 & 0 & 0 & 0 \\
\hline 6 & 0 & 0 & 0 & 0 & - \\
\hline 7 & 0 & 0 & 0 & 0 & - \\
\hline 8 & 0 & 0 & 0 & 0 & - \\
\hline 9 & 0 & 0 & 0 & 0 & - \\
\hline 10 & 0 & 0 & 0 & 0 & - \\
\hline 11 & 0 & 0 & 0 & 0 & - \\
\hline 12 & 0 & 0 & 0 & 0 & - \\
\hline 13 & 0 & 0 & 0 & 0 & - \\
\hline 14 & 0 & 0 & 0 & 0 & - \\
\hline 15 & 0 & 0 & 0 & 0 & - \\
\hline
\end{tabular}

Tab.8.7 Einzelmesswerte der Zunahme der WS Area nach 7 Tagen

\begin{tabular}{|c|c|c|c|c|c|}
\hline Nach 7 Tagen & & & & & \\
\hline Prüfkörper & $\begin{array}{l}\text { Cervitec } \\
\text { Plus }{ }^{\circledR}\end{array}$ & $\begin{array}{l}\text { Maximum } \\
\text { Cure }{ }^{\circledR}\end{array}$ & Pro Seal® & $\begin{array}{l}\text { Positiv- } \\
\text { kontrolle }\end{array}$ & $\begin{array}{l}\text { Negativ- } \\
\text { kontrolle }\end{array}$ \\
\hline 1 & 0 & 0,03 & 0,55 & 0 & 0 \\
\hline 2 & 0,01 & 0 & 0 & 0,48 & 0 \\
\hline 3 & 0,01 & 0,02 & 0 & 0 & 0 \\
\hline 4 & 0 & 0 & 0 & 0,01 & 0 \\
\hline 5 & 0 & 0 & 0,02 & 0 & 0 \\
\hline 6 & 0 & 0,11 & 0 & 0 & - \\
\hline 7 & 0 & 0 & 0 & 0,4 & - \\
\hline 8 & 0,01 & 0 & 0 & 0 & - \\
\hline 9 & 0 & 0 & 0 & 0 & - \\
\hline 10 & 0 & 0 & 0 & 0,01 & - \\
\hline 11 & 0 & 0 & 0 & 0 & - \\
\hline 12 & 0 & 0 & 0 & 0,62 & - \\
\hline 13 & 0 & 0,07 & 0 & 0,21 & - \\
\hline 14 & 0 & 0 & 0,17 & 0 & - \\
\hline 15 & 0 & 0,01 & 0 & 0 & - \\
\hline
\end{tabular}


Tab.8.8 Einzelmesswerte der Zunahme der WS Area nach 14 Tagen

\begin{tabular}{|c|c|c|c|c|c|}
\hline Nach 14 Tagen & & & & & \\
\hline Prüfkörper & $\begin{array}{l}\text { Cervitec } \\
\text { Plus }{ }^{\circledR}\end{array}$ & $\begin{array}{l}\text { Maximum } \\
\text { Cure }{ }^{\circ}\end{array}$ & Pro Sealß & $\begin{array}{l}\text { Positiv- } \\
\text { kontrolle }\end{array}$ & $\begin{array}{l}\text { Negativ- } \\
\text { kontrolle }\end{array}$ \\
\hline 1 & 0 & 0 & 0,26 & 3,81 & 0 \\
\hline 2 & 0 & 0 & 0 & 4,04 & 0 \\
\hline 3 & 3,48 & 0 & 0,34 & 0 & 0 \\
\hline 4 & 0,14 & 0 & 0 & 4,27 & 0 \\
\hline 5 & 0 & 0 & 0,01 & 2,03 & 0 \\
\hline 6 & 0 & 0,05 & 0 & 0,84 & 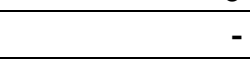 \\
\hline 7 & 0,09 & 0 & 0,1 & 2,01 & - \\
\hline 8 & 0 & 0 & 0 & 1,41 & 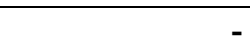 \\
\hline 9 & 0 & 0 & 0,02 & 1,63 & 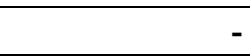 \\
\hline 10 & 0,02 & 0 & 0 & 0 & - \\
\hline 11 & 0 & 0 & 0 & 0,48 & - \\
\hline 12 & 0,03 & 0,01 & 0 & 8,77 & 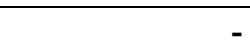 \\
\hline 13 & 0 & 0,04 & 0,01 & 2,55 & - \\
\hline 14 & 0 & 0,01 & 0,2 & 0,01 & - \\
\hline 15 & 0,03 & 0,14 & 0 & 0,02 & 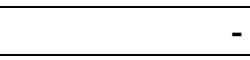 \\
\hline
\end{tabular}

Tab.8.9 Einzelmesswerte der Zunahme der WS Area nach 30 Tagen

\begin{tabular}{|c|c|c|c|c|c|}
\hline Nach 30 Tagen & & & & & \\
\hline Prüfkörper & $\begin{array}{l}\text { Cervitec } \\
\text { Plus }{ }^{\circledR}\end{array}$ & $\begin{array}{l}\text { Maximum } \\
\text { Cure }{ }^{\circ}\end{array}$ & Pro Seal® & $\begin{array}{l}\text { Positiv- } \\
\text { kontrolle }\end{array}$ & $\begin{array}{l}\text { Negativ- } \\
\text { kontrolle }\end{array}$ \\
\hline 1 & 0,05 & 0,01 & 0,05 & 10,6 & 0 \\
\hline 2 & 5,21 & 0 & 0 & 7,67 & 0 \\
\hline 3 & 5,89 & 0 & 0,56 & 7,75 & 0 \\
\hline 4 & 9,81 & 0 & 0 & 7,88 & 0 \\
\hline 5 & 0,1 & 0 & 0 & 11,6 & 0 \\
\hline 6 & 12,9 & 0,02 & 0 & 1,87 & - \\
\hline 7 & 1,52 & 0 & 0 & 3,46 & - \\
\hline 8 & 0,62 & 0 & 0 & 10,5 & - \\
\hline 9 & 0,07 & 0 & 0 & 4,6 & - \\
\hline 10 & 3,49 & 0 & 0 & 3,16 & - \\
\hline 11 & 13,1 & 0,28 & 0 & 2,7 & - \\
\hline 12 & 7,95 & 0,16 & 0 & 15,9 & - \\
\hline 13 & 7,48 & 0,01 & 0,21 & 4,73 & - \\
\hline 14 & 0,03 & 0,01 & 0,41 & 0,65 & - \\
\hline 15 & 1,86 & 0,18 & 0 & 11,5 & - \\
\hline
\end{tabular}




\subsection{Tabellenverzeichnis}

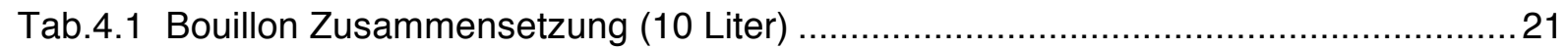

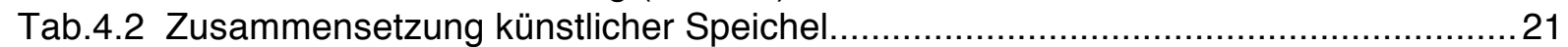

Tab.4.3 Zusammensetzung der getesteten Produkte ........................................................ 22

Tab.5.1 Prozentuale Fluoreszenzabnahme der einzelnen Gruppen zu den verschiedenen Messzeitpunkten.

Tab.5.2 p- Werte der statistischen Vergleiche der prozentualen Fluoreszenzabnahme zwischen den Kontrollgruppen und den Produktgruppen nach 30 Tagen.................36

Tab.5.3 p-Werte der statistischen Vergleiche der prozentualen Fluoreszenzabnahme zwischen den Produktgruppen nach 30 Tagen ..................................................... 37

Tab.5.4 Vergleich der WS Area zwischen den 6 Versuchsgruppen und den 4 Zeitpunkten. 38

Tab.5.5 p- Werte der statistischen Vergleiche der WS Area zwischen den Kontrollgruppen und den Produktgruppen nach 30 Tagen ........................................................... 40

Tab.5.6 p- Werte der statistischen Vergleiche der WS Area zwischen den Produktgruppen

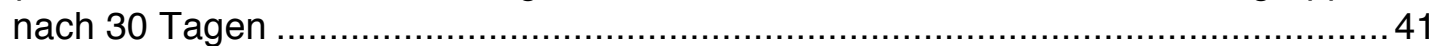

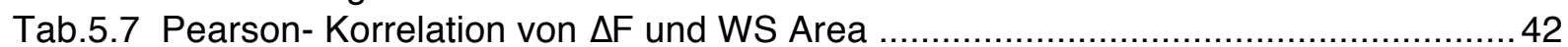

Tab.8.1 Messwerte der täglich 23 Stunden nach dem Ansetzen der Bakteriensuspension

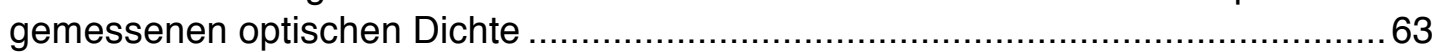

Tab.8.2 Einzelmesswerte der prozentualen Fluoreszenzabnahme zur Baseline..................64

Tab.8.3 Einzelmesswerte der prozentualen Fluoreszenzabnahme nach 7 Tagen ...............64

Tab.8.4 Einzelmesswerte der prozentualen Fluoreszenzabnahme nach 14 Tagen .............65

Tab.8.5 Einzelmesswerte der prozentualen Fluoreszenzabnahme nach 30 Tagen ............65

Tab.8.6 Einzelmesswerte der Zunahme der WS Area zur Baseline .................................66

Tab.8.7 Einzelmesswerte der Zunahme der WS Area nach 7 Tagen ................................66

Tab.8.8 Einzelmesswerte der Zunahme der WS Area nach 14 Tagen ..............................67

Tab.8.9 Einzelmesswerte der Zunahme der WS Area nach 30 Tagen .............................67

\subsection{Abbildungsverzeichnis}

Abb.2.1 Polarisationsmikroskopisches Bild einer Schmelzkaries ..................................... 6

Abb.2.2 White- Spot- Läsionen eines kieferorthopädisch behandelten Patienten nach

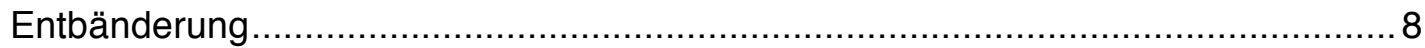

Abb.2.3 Auswertungsbereich eines Prüfkörpers nach der QLF-Analyse ............................16

Abb.3.1 Übersicht über den Versuchsablauf............................................................... 19

Abb.4.1 Separierung des Zahnes zur Gewinnung von zwei Kronenhälften ........................23

Abb.4.2 Ausstanzen der Kronenhälfte und resultierende Schmelzprobe ............................24

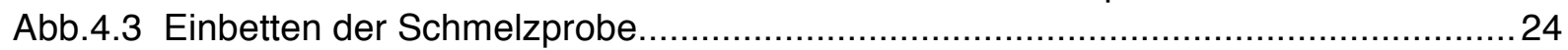

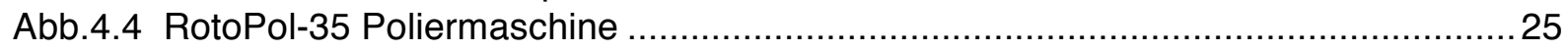

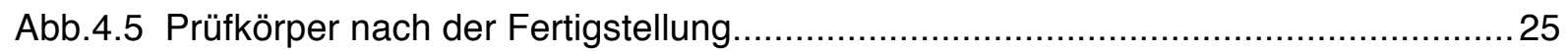

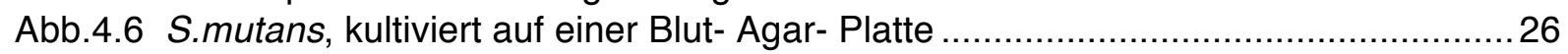

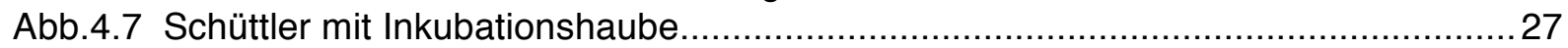

Abb.4.8 Grafische Darstellung der Wachstumskurve ...................................................... 28

Abb.4.9 QLF- Apparatur mit PC, angeschlossenem QLF- Auslesegerät, Kamera und

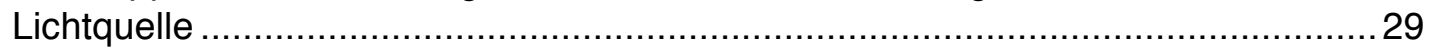

Abb.4.10 Schraubdeckeldose, verschlossen und ohne Deckel mit darin gelagerten Prüfkörpern

Abb.5.1 Box- Plot der prozentualen Fluoreszenzabnahme im Vergleich zwischen Baseline und dem Messzeitpunkt nach 30 Tagen der einzelnen Gruppen........................... 35

Abb.5.2 Box- Plot der prozentualen Fluoreszenzabnahme der verschiedenen Gruppen nach 30 Tagen ....

Abb.5.3 Box- Plot der Vergleiche zwischen Baseline und dem Messzeitpunkt nach 30 Tagen 


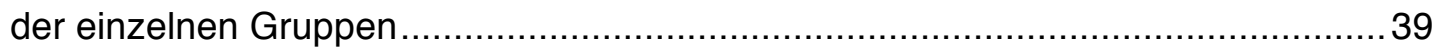

Abb.5.4 Box- Plot der WS Area der verschiedenen Gruppen nach 30 Tagen ...................... 40

Abb.5.5 Grafische Darstellung der Korrelation von prozentualer Fluoreszenzabnahme und WS Area aller Gruppen unabhängig von der Zeit .............................................. 42

Abb.5.6 QLF- Bilder zu den verschiedenen Messzeitpunkten ...................................... 44 


\section{Literaturverzeichnis}

Aas JA, Paster BJ, Stokes LN, Olsen I, Dewhirst FE (2005): Defining the normal bacterial flora of the oral cavity. J Clin Microbiol $\underline{43}, 5721-5732$

Alexander SA, Ripa LW (2000): Effects of self-applied topical fluoride preparations in orthodontic patients. Angle Orthod $\underline{70}, 424-430$

Alves PV, Alviano WS, Bolognese AM, Nojima LI (2008): Treatment protocol to control Streptococcus mutans level in an orthodontic patient with high caries risk. Am J Orthod Dentofacial Orthop 133, 91-94

Ando M, Eckert GJ, Stookey GK, Zero DT (2004): Effect of imaging geometry on evaluating natural white-spot lesions using quantitative light-induced fluorescence. Caries Res 38 , 39-44

Apostolopoulos N, Caumanns J, Fungk C, Geukes A: Statistik Interaktiv!: Deskriptive Statistik, Springer, Berlin 2002

Arends J, ten Bosch JJ (1992): Demineralization and remineralization evaluation techniques. J Dent Res 71 Spec No, 924-928

Arnold WH, Gaengler P, Sabov K, Schmitz I, Gedalia I, Steinberg D (2001): Induction and 3D reconstruction of caries-like lesions in an experimental dental plaque biofilm model. J Oral Rehabil 28, 748-754

Artun J, Brobakken BO (1986): Prevalence of carious white spots after orthodontic treatment with multibonded appliances. Eur J Orthod 8, 229-234

Arweiler N, Mombelli A, Welk A, Zimmer S, Scholz V (2011): 40 Jahre Chlorhexidin in der klinischen Forschung. ZWR Dtsch Zahnärztebl 120, 318-323

Attin T, Hartmann O, Hilgers RD, Hellwig E (1995): Fluoride retention of incipient enamel lesions after treatment with a calcium fluoride varnish in vivo. Arch Oral Biol $\underline{40}, 169-174$

Attin R, Thon C, Schlagenhauf U, Werner C, Wiegand A, Hannig C, Attin T (2005):

Recolonization of mutans steptococci on teeth with orthodontic appliances after antimicrobial therapy. Eur J Orthod 27, 489-493

Autio-Gold J (2008): The role of chlorhexidine in caries prevention. Oper Dent $\underline{33}, 710-716$ 
Balenseifen JW, Madonia JV (1970): Study of dental plaque in orthodontic patients. J Dent Res $\underline{49}, 320-324$

Banks PA, Richmond S (1994): Enamel sealants: a clinical evaluation of their value during fixed appliance therapy. Eur J Orthod 16, 19-25

Basch E, Ulbricht C, Hammerness P, Bevins A, Sollars D (2004): Thyme (Thymus vulgaris L.), thymol. J Herb Pharmacother 4 , 49-67

Basdra EK, Huber H, Komposch G (1996): Fluoride released from orthodontic bonding agents alters the enamel surface and inhibits enamel demineralization in vitro. Am $\mathrm{J}$ Orthod Dentofacial Orthop 109, 466-472

Behnan SM, Arruda AO, Gonzalez-Cabezas C, Sohn W, Peters MC (2010): In-vitro evaluation of various treatments to prevent demineralization next to orthodontic brackets. Am J Orthod Dentofacial Orthop 138, 712 e711-717; discussion 712-713

Benham AW, Campbell PM, Buschang PH (2009): Effectiveness of pit and fissure sealants in reducing white spot lesions during orthodontic treatment. A pilot study. Angle Orthod 79 , 338345

Benson PE, Parkin N, Millett DT, Dyer FE, Vine S, Shah A (2004): Fluorides for the prevention of white spots on teeth during fixed brace treatment. Cochrane Database Syst Rev CD003809

Bergstrand F, Twetman S (2011): A review on prevention and treatment of post-orthodontic white spot lesions - evidence-based methods and emerging technologies. Open Dent $\mathrm{J} \underline{5}$, 158-162

Bonesvoll P, Lokken P, Rolla G (1974): Influence of concentration, time, temperature and pH on the retention of chlorhexidine in the human oral cavity after mouth rinses. Arch Oral Biol 19 , 1025-1029

Brightman LJ, Terezhalmy GT, Greenwell H, Jacobs M, Enlow DH (1991): The effects of a $0.12 \%$ chlorhexidine gluconate mouthrinse on orthodontic patients aged 11 through 17 with established gingivitis. Am J Orthod Dentofacial Orthop 100, 324-329

Buchalla W (2008): Stimmt unser Weltbild der Karies noch? Quintessenz 59, 1243-1249

Buren JL, Staley RN, Wefel J, Qian F (2008): Inhibition of enamel demineralization by an enamel sealant, Pro Seal: an in-vitro study. Am J Orthod Dentofacial Orthop 133, 88-94 
Busscher HJ, van der Mei HC (1997): Physico-chemical interactions in initial microbial adhesion and relevance for biofilm formation. Adv Dent Res 11, 24-32

Buzalaf MA, Hannas AR, Magalhaes AC, Rios D, Honorio HM, Delbem AC (2010): pHcycling models for in vitro evaluation of the efficacy of fluoridated dentifrices for caries control: strengths and limitations. J Appl Oral Sci 18, 316-334

Cain K, Hicks J, English J, Flaitz C, Powers JM, Rives T (2006): In vitro enamel caries formation and orthodontic bonding agents. Am J Dent 19, 187-192

Chang HS, Walsh LJ, Freer TJ (1997): Enamel demineralization during orthodontic treatment. Aetiology and prevention. Aust Dent J 42, 322-327

Chapman JA, Roberts WE, Eckert GJ, Kula KS, Gonzalez-Cabezas C (2010): Risk factors for incidence and severity of white spot lesions during treatment with fixed orthodontic appliances. Am J Orthod Dentofacial Orthop 138, 188-194

Cohen WJ, Wiltshire WA, Dawes C, Lavelle CL (2003): Long-term in vitro fluoride release and rerelease from orthodontic bonding materials containing fluoride. Am J Orthod Dentofacial Orthop 124, 571-576

Coreil MN, McInnes-Ledoux P, Ledoux WR, Weinberg R (1990): Shear bond strength of four orthodontic bonding systems. Am J Orthod Dentofacial Orthop 97, 126-129

Davies A (1973): The mode of action of chlorhexidine. J Periodontal Res Suppl 12, 68-75

de Josselin de Jong E, Sundstrom F, Westerling H, Tranaeus S, ten Bosch JJ, AngmarMansson B (1995): A new method for in vivo quantification of changes in initial enamel caries with laser fluorescence. Caries Res 29, 2-7

Demito CF, Vivaldi-Rodrigues G, Ramos AL, Bowman SJ (2004): The efficacy of a fluoride varnish in reducing enamel demineralization adjacent to orthodontic brackets: an in vitro study. Orthod Craniofac Res $\underline{7}, 205-210$

Denbesten P, Li W (2011): Chronic fluoride toxicity: dental fluorosis. Monogr Oral Sci 22, 8196

Denes J, Gabris K (1991): Results of a 3-year oral hygiene programme, including amine fluoride products, in patients treated with fixed orthodontic appliances. Eur J Orthod 13, 129133 
Deng DM, ten Cate JM (2004): Demineralization of dentin by Streptococcus mutans biofilms grown in the constant depth film fermentor. Caries Res $\underline{38}, 54-61$

DGZMK (2000): Empfehlungen zur Kariesprophylaxe mit Fluoriden. Wissenschaftliche Stellungnahme (Gülzow HJ, Hellwig E, Hetzer G), URL: http://www.dgzmk.de

DGZMK (2007): Leitlinie Fluoridierungsmaßnahmen. (Gülzow HJ, Hellwig, E, Hetzer G), URL: http://www.dgzmk.de

Dummer PM, Edmunds DH, Green RM (1982): Demineralisation of human enamel by Streptococcus mutans NCTC 10832 using a sequential batch culture technique. Caries Res 16, $193-196$

Edmunds DH, Whittaker DK, Green RM (1988): Suitability of human, bovine, equine, and ovine tooth enamel for studies of artificial bacterial carious lesions. Caries Res 22, 327-336

Emilson CG (1994): Potential efficacy of chlorhexidine against mutans streptococci and human dental caries. J Dent Res $\underline{73}, 682-691$

Emilson CG, Krasse B (1985): Support for and implications of the specific plaque hypothesis. Scand J Dent Res 93, 96-104

Enaia M, Bock N, Ruf S (2011): White-spot lesions during multibracket appliance treatment: A challenge for clinical excellence. Am J Orthod Dentofacial Orthop 140, e17-24

Farhadian N, Miresmaeili A, Eslami B, Mehrabi S (2008): Effect of fluoride varnish on enamel demineralization around brackets: an in-vivo study. Am J Orthod Dentofacial Orthop 133, S95-98

Featherstone JD (2000): The science and practice of caries prevention. J Am Dent Assoc 131, 887-899

Featherstone JD, Mellberg JR (1981): Relative rates of progress of artificial carious lesions in bovine, ovine and human enamel. Caries Res 15, 109-114

Fejerskov O, Ekstrand J, Burt BA: Fluoride in dentistry, Munksgaard, Kopenhagen 1996

Fischer C, Lussi, A., Hotz, P. (1994): Kariostatische Wirkungsmechanismen der Fluoride. Schweiz Monatsschr Zahnmed 105, 311-317 
Fitzgerald RJ, Keyes PH (1960): Demonstration of the etiologic role of streptococci in experimental caries in the hamster. J Am Dent Assoc 61, 9-19

Fonseca RB, Haiter-Neto F, Carlo HL, Soares CJ, Sinhoreti MA, Puppin-Rontani RM, CorrerSobrinho $L$ (2008): Radiodensity and hardness of enamel and dentin of human and bovine teeth, varying bovine teeth age. Arch Oral Biol $\underline{53}, 1023-1029$

Fontana M, Dunipace AJ, Gregory RL, Noblitt TW, Li Y, Park KK, Stookey GK (1996): An in vitro microbial model for studying secondary caries formation. Caries Res $\underline{30}, 112-118$

Franke G, Baume LJ (1976): Klassifizierung Epidemiologischer Studien Über Zahnkaries Und Definition Verwandter Begriffe, Herausgegeben von Mitgliedern der Arbeitsgruppe 1 der Kommission zur Klassifizierung und Normung von Statistiken über Gebissbefunde der Fédération Dentaire Internationale (FDI). Int Dent J 26, 73-95

Gängler P, Hoffmann, T., Willershausen, B., Schwenzer, N., Ehrenfeld, M.: Zahn-MundKiefer-Heilkunde. Lehrbuchreihe zur Aus- und Weiterbildung: Konservierende Zahnheilkunde und Parodontologie, Thieme, Stuttgart 2010

Geiger AM, Gorelick L, Gwinnett AJ, Griswold PG (1988): The effect of a fluoride program on white spot formation during orthodontic treatment. Am J Orthod Dentofacial Orthop 93, 29-37

Geiger AM, Gorelick L, Gwinnett AJ, Benson BJ (1992): Reducing white spot lesions in orthodontic populations with fluoride rinsing. Am J Orthod Dentofacial Orthop 101, 403-407

George AM, Kalangi SK, Vasudevan M, Krishnaswamy NR (2010): Chlorhexidine varnishes effectively inhibit Porphyromonas gingivalis and Streptococcus mutans - an in vivo study. J Indian Soc Periodontol 14, 178-180

Gmur R, Giertsen E, van der Veen MH, de Josselin de Jong E, ten Cate JM, Guggenheim B (2006): In vitro quantitative light-induced fluorescence to measure changes in enamel mineralization. Clin Oral Investig 10, 187-195

Gorelick L, Geiger AM, Gwinnett AJ (1982): Incidence of white spot formation after bonding and banding. Am J Orthod 81, 93-98

Gorton J, Featherstone JD (2003): In vivo inhibition of demineralization around orthodontic brackets. Am J Orthod Dentofacial Orthop 123, 10-14

Guggenheim B, Giertsen E, Schupbach P, Shapiro S (2001): Validation of an in vitro biofilm model of supragingival plaque. J Dent Res $\underline{80}, 363-370$ 
Gustafsson BE, Quensel CE, Lanke LS, Lundqvist C, Grahnen H, Bonow BE, Krasse B (1954): The Vipeholm dental caries study; the effect of different levels of carbohydrate intake on caries activity in 436 individuals observed for five years. Acta Odontol Scand 11, 232-264

Hadler-Olsen S, Sandvik K, El-Agroudi MA, Ogaard B (2011): The incidence of caries and white spot lesions in orthodontically treated adolescents with a comprehensive caries prophylactic regimen-a prospective study. Eur J Orthod [Epub ahead of print]

Hayati F, Okada A, Kitasako Y, Tagami J, Matin K (2011): An artificial biofilm induced secondary caries model for in vitro studies. Aust Dent J $\underline{56}, 40-47$

Hellwig E (1996): Fluoride - Chemie und Biochemie. Dtsch Zahnarztl Z 51, 638-648

Hellwig E, Klimek J, Attin T: Einführung in die Zahnerhaltung, Deutscher Ärzte-Verlag, Köln 2009

Hess E, Campbell PM, Honeyman AL, Buschang PH (2011): Determinants of enamel decalcification during simulated orthodontic treatment. Angle Orthod 81, 836-842

Hjeljord LG, Rolla G, Bonesvoll P (1973): Chlorhexidine-protein interactions. J Periodontal Res Suppl 12, 11-16

Hotz PR (1996): Anwendung der Fluoride in der Zahnmedizin. Dtsch Zahnärztl Z 51, 649-653

Hu W, Featherstone JD (2005): Prevention of enamel demineralization: an in-vitro study using light-cured filled sealant. Am J Orthod Dentofacial Orthop 128, 592-600; quiz 670

Ifland S, Kühnisch J, Heinrich-Weltzien R (2004): Die quantitative lichtinduzierte Fluoreszenz (QLF) - Eine nichtinvasive Methode zur Kariesdiagnostik. ZWR Dtsch Zahnärztebl 113, 254258

Inspektor Research Systems BV: URL: http://www.inspektor.nl/ 2000

Ismail Al, Hasson $\mathrm{H}$ (2008): Fluoride supplements, dental caries and fluorosis: a systematic review. J Am Dent Assoc 139, 1457-1468

James P, Parnell C, Whelton H (2010): The caries-preventive effect of chlorhexidine varnish in children and adolescents: a systematic review. Caries Res $44,333-340$ 
Jenkins S, Addy M, Newcombe RG (1994): Dose response of chlorhexidine against plaque and comparison with triclosan. J Clin Periodontol 21, 250-255

Joseph VP, Rossouw PE, Basson NJ (1994): Some "sealants" seal--a scanning electron microscopy (SEM) investigation. Am J Orthod Dentofacial Orthop 105, 362-368

Karlinsey RL, Mackey AC, Walker TJ, Frederick KE, Blanken DD, Flaig SM, Walker ER (2011): In vitro remineralization of human and bovine white-spot enamel lesions by $\mathrm{NaF}$ dentifrices: A pilot study. J Dent Oral Hyg $\underline{3}, 22-29$

Keim RG (2011): Preventing and treating white-spot lesions. J Clin Orthod 45, 9-10

Keyes PH (1962): Recent advances in dental caries research. Int Dent J 12, 443-463

Keyes PH, Fitzgerald RJ (1962): Dental caries in the Syrian hamster. IX. Arch Oral Biol $\underline{7}$, 267-277

Kneist S, Zingler S, Lux C (2008): Therapiebegleitende Maßnahmen zur Kontrolle des Karies- und Demineralisationsrisikos bei kieferorthopädischen Patienten. ZWR Dtsch Zahnärztebl 117, 218-226

König KG: Karies und Kariesprophylaxe, Goldmann, München 1971

König KG (1993): Role of fluoride toothpastes in a caries-preventive strategy. Caries Res 27 Suppl 1, 23-28

Krasse B (1966): Human streptococci and experimental caries in hamsters. Arch Oral Biol 11, 429-436

Kukleva MP, Shetkova DG, Beev VH (2002): Comparative age study of the risk of demineralization during orthodontic treatment with brackets. Folia Med (Plovdiv) $\underline{44}, 56-59$

Limeback H (1994): Enamel formation and the effects of fluoride. Community Dent Oral Epidemiol 22, 144-147

Lippert F, Butler A, Lynch RJ, Hara AT (2012): Effect of Fluoride, Lesion Baseline Severity and Mineral Distribution on Lesion Progression. Caries Res $\underline{46}, 23-30$

Loe H, Schiott CR (1970): The effect of mouthrinses and topical application of chlorhexidine on the development of dental plaque and gingivitis in man. J Periodontal Res $\underline{5}, 79-83$ 
Lovrov S, Hertrich K, Hirschfelder U (2007): Enamel Demineralization during Fixed Orthodontic Treatment - Incidence and Correlation to Various Oral-hygiene Parameters. J Orofac Orthop 68, 353-363

Lundstrom F, Krasse B (1987): Streptococcus mutans and lactobacilli frequency in orthodontic patients; the effect of chlorhexidine treatments. Eur J Orthod $\underline{9}, 109-116$

Madlena M, Vitalyos G, Marton S, Nagy G (2000): Effect of chlorhexidine varnish on bacterial levels in plaque and saliva during orthodontic treatment. J Clin Dent 11, 42-46

Marsh P, Martin MV: Orale Mikrobiologie, Thieme, Stuttgart 2003

Marsh PD (1994): Microbial ecology of dental plaque and its significance in health and disease. Adv Dent Res $\underline{8}$, 263-271

Marsh PD (2006): Dental plaque as a biofilm and a microbial community - implications for health and disease. BMC Oral Health 6 Suppl 1, S14

Marsh PD, Bowden GH (1995): The role of microbiology in models of dental caries. Adv Dent Res $\underline{9}$, 244-254; discussion 255-269

Marsh PD, Moter A, Devine DA (2011): Dental plaque biofilms: communities, conflict and control. Periodontol $2000 \underline{55}, 16-35$

McNeill CJ, Wiltshire WA, Dawes C, Lavelle CL (2001): Fluoride release from new light-cured orthodontic bonding agents. Am J Orthod Dentofacial Orthop 120, 392-397

Mellberg JR (1992): Hard-tissue substrates for evaluation of cariogenic and anti-cariogenic

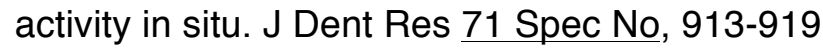

Miller WD: Die Mikroorganismen der Mundhöhle, die örtlichen und allgemeinen Erkrankungen, welche durch dieselben hervorgerufen werden, Georg Thieme Verlag, Leipzig 1889

Mitchell L (1992): Decalcification during orthodontic treatment with fixed appliances--an overview. Br J Orthod 19, 199-205

Mizrahi E (1982): Enamel demineralization following orthodontic treatment. Am J Orthod 82 , 62-67 
Nakata K, Nikaido T, Ikeda M, Foxton RM, Tagami J (2009): Relationship between fluorescence loss of QLF and depth of demineralization in an enamel erosion model. Dent Mater J 28, 523-529

Ogaard B (1989): Prevalence of white spot lesions in 19-year-olds: a study on untreated and orthodontically treated persons 5 years after treatment. Am J Orthod Dentofacial Orthop 96, 423-427

Ogaard B, Rolla G, Arends J (1988a): Orthodontic appliances and enamel demineralization. Part 1. Lesion development. Am J Orthod Dentofacial Orthop 94, 68-73

Ogaard B, Rolla G, Arends J, ten Cate JM (1988b): Orthodontic appliances and enamel demineralization. Part 2. Prevention and treatment of lesions. Am J Orthod Dentofacial Orthop $94,123-128$

Ogaard B, Rolla G, Ruben J, Dijkman T, Arends J (1988c): Microradiographic study of demineralization of shark enamel in a human caries model. Scand J Dent Res 96, 209-211

Ogaard B, Seppa L, Rolla G (1994): Professional topical fluoride applications--clinical efficacy and mechanism of action. Adv Dent Res $\underline{8}, 190-201$

Ogaard B, Duschner H, Ruben J, Arends J (1996): Microradiography and confocal laser scanning microscopy applied to enamel lesions formed in vivo with and without fluoride varnish treatment. Eur J Oral Sci 104, 378-383

Ogaard B, Larsson E, Glans R, Henriksson T, Birkhed D (1997): Antimicrobial effect of a chlorhexidine-thymol varnish (Cervitec) in orthodontic patients. A prospective, randomized clinical trial. J Orofac Orthop $\underline{58,206-213}$

Ogaard B, Larsson E, Henriksson T, Birkhed D, Bishara SE (2001): Effects of combined application of antimicrobial and fluoride varnishes in orthodontic patients. Am J Orthod Dentofacial Orthop 120, 28-35

Ogaard B, Alm AA, Larsson E, Adolfsson U (2006): A prospective, randomized clinical study on the effects of an amine fluoride/stannous fluoride toothpaste/mouthrinse on plaque, gingivitis and initial caries lesion development in orthodontic patients. Eur J Orthod 28, 8-12

O'Reilly MM, Featherstone JD (1987): Demineralization and remineralization around orthodontic appliances: an in vivo study. Am J Orthod Dentofacial Orthop 92, 33-40 
Orland FJ, Blayney JR, Harrison RW, Reyniers JA, Trexler PC, Wagner M, Gordon HA, Luckey TD (1954): Use of the germfree animal technic in the study of experimental dental caries. I. Basic observations on rats reared free of all microorganisms. J Dent Res $33,147-$ 174

Orland FJ, Blayney JR, Harrison RW, Reyniers JA, Trexler PC, Ervin RF, Gordon HA, Wagner M (1955): Experimental caries in germfree rats inoculated with enterococci. J Am Dent Assoc 50, 259-272

Petersson LG (1993): Fluoride mouthrinses and fluoride varnishes. Caries Res 27 Suppl 1, $35-42$

Petersson LG, Magnusson K, Andersson H, Almquist B, Twetman S (2000): Effect of quarterly treatments with a chlorhexidine and a fluoride varnish on approximal caries in caries-susceptible teenagers: a 3-year clinical study. Caries Res 34, 140-143

Petersson LG, Twetman S, Dahlgren H, Norlund A, Holm AK, Nordenram G, Lagerlof F, Soder B, Kallestal C, Mejare I (2004): Professional fluoride varnish treatment for caries control: a systematic review of clinical trials. Acta Odontol Scand 62, 170-176

Pinar Erdem A, Sepet E, Kulekci G, Trosola SC, Guven Y (2012): Effects of Two Fluoride Varnishes and One Fluoride/Chlorhexidine Varnish on Streptococcus mutans and Streptococcus sobrinus Biofilm Formation in Vitro. Int J Med Sci $\underline{9}$, 129-136

Pretty IA, Edgar WM, Higham SM (2002): The effect of ambient light on QLF analyses. J Oral Rehabil 29, 369-373

Pretty IA, Pender N, Edgar WM, Higham SM (2003): The in vitro detection of early enamel de- and re-mineralization adjacent to bonded orthodontic cleats using quantitative lightinduced fluorescence. Eur J Orthod 25, 217-223

Pretty IA, Edgar WM, Higham SM (2004): The effect of dehydration on quantitative lightinduced fluorescence analysis of early enamel demineralization. J Oral Rehabil $31,179-184$

Pretty IA, Ellwood PG, Davies RM, Worthington HW, Ellwood RP (2006): The effects of illumination and focal distance on light-induced fluorescence images in vitro. Caries Res $\underline{40}$, 73-76

Pseiner BC (2008): Fluoridprophylaxe in der Therapie mit festsitzenden Apparaturen - eine Literaturübersicht. Inf Orthod Kieferorthop 40, 233-237 
Rao A, Malhotra N (2011): The role of remineralizing agents in dentistry: a review. Compend Contin Educ Dent 32, 26-33; quiz 34, 36

Richter AE, Arruda AO, Peters MC, Sohn W (2011): Incidence of caries lesions among patients treated with comprehensive orthodontics. Am J Orthod Dentofacial Orthop 139, 657664

Ruppert M, Schlagenhauf U (2004): Chlorhexidin in der Zahnheilkunde. Quintessenz 55, 5565

Salar DV, Garcia-Godoy F, Flaitz CM, Hicks MJ (2007): Potential inhibition of demineralization in vitro by fluoride-releasing sealants. J Am Dent Assoc 138, 502-506

Schiffner U, Schmeiser, R. (1995): Eine Methode zur bakteriellen Erzeugung von Wurzelkaries in einer künstlichen Mundhöhle. Dtsch Zahnarztl Z 50, 45-48

Schiiott CR, Loe H, Briner WW (1976): Two year oral use of chlorhexidine in man IV. Effect on various medical parameters. J Periodontal Res 11, 158-164

Schroeder HE: Pathobiologie Oraler Strukturen: Zaehne, Pulpa, Parodont, Karger - Verlag, Freiburg 1997

Seemann R, Bizhang M, Kluck I, Loth J, Roulet JF (2005): A novel in vitro microbial-based model for studying caries formation - development and initial testing. Caries Res $\underline{39}, 185-190$

Seemann R, Kluck I, Kage A (2006): An in vitro microbial-based model for studying cariespreventive agents. Acta Odontol Scand 64, 27-30

Shapiro S, Giertsen E, Guggenheim B (2002): An in vitro oral biofilm model for comparing the efficacy of antimicrobial mouthrinses. Caries Res $\underline{36}$, 93-100

Silverstone LM: Dental caries: aetiology, pathology and prevention, Macmillan, London 1981

Sissons CH (1997): Artificial dental plaque biofilm model systems. Adv Dent Res 11, 110-126

Soliman MM, Bishara SE, Wefel J, Heilman J, Warren JJ (2006): Fluoride release rate from an orthodontic sealant and its clinical implications. Angle Orthod $\underline{76}, 282-288$

Splieth $\mathrm{CH}$, Treuner A, Gedrange T, Berndt C (2011): Caries-preventive and remineralizing effect of fluoride gel in orthodontic patients after 2 years. Clin Oral Investig 16, 1395-1399 
Stephan RM (1940): Changes in hydrogen-ion concentration on tooth surfaces and in caries lesions. J Am Dent Assoc 27, 718-723

Sudjalim TR, Woods MG, Manton DJ, Reynolds EC (2007): Prevention of demineralization around orthodontic brackets in vitro. Am J Orthod Dentofacial Orthop 131, 705 e701-709

Takeshita EM, Exterkate RA, Delbem AC, ten Cate JM (2011): Evaluation of different fluoride concentrations supplemented with trimetaphosphate on enamel de- and remineralization in vitro. Caries Res $\underline{45}$, 494-497

Tanaka JL, Medici Filho E, Salgado JA, Salgado MA, Moraes LC, Moraes ME, Castilho JC (2008): Comparative analysis of human and bovine teeth: radiographic density. Braz Oral Res 22, 346-351

Tang G, Yip HK, Cutress TW, Samaranayake LP (2003): Artificial mouth model systems and their contribution to caries research: a review. J Dent 31, 161-171

Tanna N, Kao E, Gladwin M, Ngan PW (2009): Effects of sealant and self-etching primer on enamel decalcification. Part I: an in-vitro study. Am J Orthod Dentofacial Orthop 135, 199205

Ten Bosch JJ, Angmar-Mansson B (1991): A review of quantitative methods for studies of mineral content of intra-oral caries lesions. J Dent Res $\underline{70}, 2-14$

ten Cate JM, Duijsters PP (1982): Alternating demineralization and remineralization of artificial enamel lesions. Caries Res 16, 201-210

ten Cate JM, Duijsters PP (1983): Influence of fluoride in solution on tooth demineralization. II. Microradiographic data. Caries Res $\underline{17}, 513-519$

ten Cate JM, Featherstone JD (1991): Mechanistic aspects of the interactions between fluoride and dental enamel. Crit Rev Oral Biol Med 2, 283-296

ten Cate JM, Exterkate RA, Buijs MJ (2006): The relative efficacy of fluoride toothpastes assessed with $\mathrm{pH}$ cycling. Caries Res $\underline{40}, 136-141$

Topaloglu-Ak A, Ertugrul F, Eden E, Ates M, Bulut H (2011): Effect of orthodontic appliances on oral microbiota--6 month follow-up. J Clin Pediatr Dent 35, 433-436

Tranaeus S, Shi XQ, Angmar-Mansson B (2005): Caries risk assessment: methods available to clinicians for caries detection. Community Dent Oral Epidemiol 33, 265-273 
Travess H, Roberts-Harry D, Sandy J (2004): Orthodontics. Part 6: Risks in orthodontic treatment. Br Dent J 196, 71-77

Tufekci E, Dixon JS, Gunsolley JC, Lindauer SJ (2011): Prevalence of white spot lesions during orthodontic treatment with fixed appliances. Angle Orthod 81, 206-210

Twetman S, Hallgren A, Petersson LG (1995): Effect of an antibacterial varnish on mutans streptococci in plaque from enamel adjacent to orthodontic appliances. Caries Res $\underline{29}, 188-$ 191

van der Veen MH, de Josselin de Jong E (2000): Application of quantitative light-induced fluorescence for assessing early caries lesions. Monogr Oral Sci 17, 144-162

Van Loveren C (1990): The antimicrobial action of fluoride and its role in caries inhibition. J Dent Res 69 Spec No, 676-681; discussion 682-673

Vieira A, Ruben JL, Huysmans MC (2005): Effect of titanium tetrafluoride, amine fluoride and fluoride varnish on enamel erosion in vitro. Caries Res 39, 371-379

Wenisch T: Kurzlehrbuch Physik, Chemie, Biologie, Urban \& Fischer bei Elsevier, München 2009

White DJ (1995): The application of in vitro models to research on demineralization and remineralization of the teeth. Adv Dent Res $\underline{9}$, 175-193; discussion 194-177

Willmot DR (2004): White lesions after orthodontic treatment: does low fluoride make a difference? J Orthod 31, 235-242; discussion 202

Wiltshire WA, Janse van Rensburg SD (1995): Fluoride release from four visible light-cured orthodontic adhesive resins. Am J Orthod Dentofacial Orthop 108, 278-283

Wu J, Donly ZR, Donly KJ, Hackmyer S (2010): Demineralization Depth Using QLF and a Novel Image Processing Software. Int J Dent 2010, 958264

Yassen GH, Platt JA, Hara AT (2011): Bovine teeth as substitute for human teeth in dental research: a review of literature. J Oral Sci $\underline{53}, 273-282$

Zachrisson BU (1976): Cause and prevention of injuries to teeth and supporting structures during orthodontic treatment. Am J Orthod 69 , 285-300 
Zaura-Arite E, ten Cate JM (2000): Effects of fluoride- and chlorhexidine-containing varnishes on plaque composition and on demineralization of dentinal grooves in situ. Eur J Oral Sci $\underline{108,}$ 154-161

Zentner A, Duschner H (1996): Structural changes of acid etched enamel examined under confocal laser scanning microscope. J Orofac Orthop 57, 202-209

Zimmer BW, Rottwinkel Y (2004): Assessing patient-specific decalcification risk in fixed orthodontic treatment and its impact on prophylactic procedures. Am J Orthod Dentofacial Orthop 126, 318-324 


\section{Danksagung}

An erster Stelle danke ich meinem Doktorvater, Herrn Prof. Dr. med. dent. Rainer F. Mausberg, für die freundliche Überlassung des Themas und die konstruktive Unterstützung.

Mein besonderer Dank gilt Herrn Dr. med. dent. Dirk Ziebolz für die hervorragende Betreuung, die fachlichen Anregungen, die unermüdliche Tatkraft und stetige Motivation zu jedem Zeitpunkt dieser Arbeit.

Bedanken möchte ich mich weiterhin bei Frau Monika Hoch für die geduldige Einarbeitung im Labor und die zuverlässige Betreuung während der Versuchsphase.

Für die freundliche Überlassung des Laborarbeitsplatzes danke ich dem Institut für Medizinische Mikrobiologie Zentrum Hygiene und Humangenetik der Universitätsmedizin Göttingen.

Ein weiterer Dank gilt den Mitarbeitern der Abteilung Medizinische Statistik der Universitätsmedizin Göttingen, Dr. Klaus Jung, Simon Schneider und Thomas Asendorf, für ihre geduldige Hilfestellung bei der Auswertung der Studienergebnisse.

Auch möchte ich mich bei all denen bedanken, die mich auf dem Weg des forschenden Arbeitens menschlich unterstützt, motiviert und begleitet haben. Besonders bedanken möchte ich mich bei Clarissa Milmann für die vielen gemeinsamen Stunden während der Versuchsphase. 


\section{Lebenslauf}

Am 05.Dezember 1985 wurde ich als viertes Kind meiner Eltern, Dr. med. dent. Hans- Peter Kroker und Gabriele Kroker, geb. Knapstein, in Meppen (Niedersachsen) geboren.

Von 1992 bis 1996 besuchte ich die Grundschule Hemsen, von 1996 bis 2005 war ich Schülerin am Gymnasium Marianum in Meppen. Dort erlangte ich im Juni 2005 die allgemeine Hochschulreife. Im April 2006 nahm ich das Studium der Zahnheilkunde an der Georg- August- Universität in Göttingen auf. Die naturwissenschaftliche Vorprüfung legte ich im Februar 2007 ab, und die zahnärztliche Vorprüfung absolvierte ich im September 2008. Im Juni 2011 bestand ich mein Staatsexamen und erhielt die Approbation als Zahnärztin.

Im Wintersemester 2011/2012 arbeitete ich als Lehrbeauftragte im Phantomkurs der Zahnerhaltungskunde der Abteilung für Präventive Zahnmedizin, Parodontologie und Kariologie der Universitätsmedizin Göttingen. Seit dem 1. April 2012 arbeite ich als Zahnärztin in der Zahnarztpraxis von Herrn Horst Wendorff M.Sc. in Berlin. 